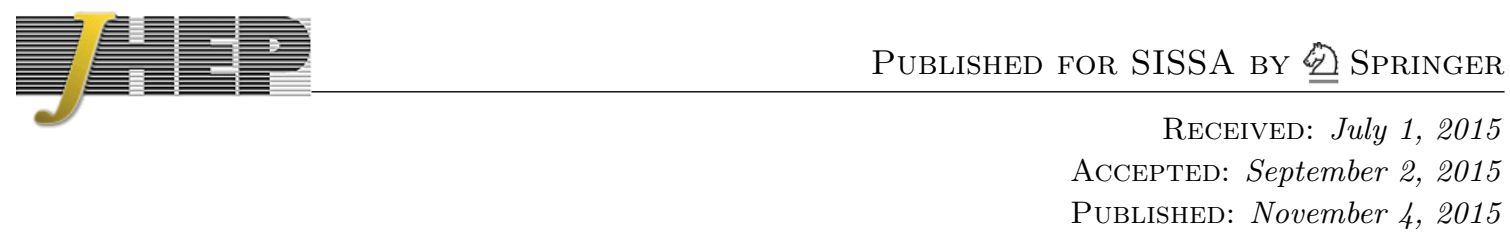

\title{
Flavor from the electroweak scale
}

\author{
Martin Bauer, ${ }^{a, d}$ Marcela Carena $^{a, b, c}$ and Katrin Gemmler ${ }^{a}$ \\ ${ }^{a}$ Fermilab, \\ P.O. Box 500, Batavia, IL 60510, U.S.A. \\ ${ }^{b}$ Enrico Fermi Institute, University of Chicago, \\ Chicago, IL 6063\%, U.S.A. \\ ${ }^{c}$ Kavli Institute for Cosmological Physics, University of Chicago, \\ Chicago, IL 6063\%, U.S.A. \\ ${ }^{d}$ Institut für Theoretische Physik, Universität Heidelberg, \\ 69120 Heidelberg, Germany \\ E-mail: m.bauer@thphys.uni-heidelberg.de, carena@fnal.gov, \\ katrin@fnal.gov
}

ABSTRACT: We discuss the possibility that flavor hierarchies arise from the electroweak scale in a two Higgs doublet model, in which the two Higgs doublets jointly act as the flavon. Quark masses and mixing angles are explained by effective Yukawa couplings, generated by higher dimensional operators involving quarks and Higgs doublets. Modified Higgs couplings yield important effects on the production cross sections and decay rates of the light Standard Model like Higgs. In addition, flavor changing neutral currents arise at tree-level and lead to strong constraints from meson-antimeson mixing. Remarkably, flavor constraints turn out to prefer a region in parameter space that is in excellent agreement with the one preferred by recent Higgs precision measurements at the Large Hadron Collider (LHC). Direct searches for extra scalars at the LHC lead to further constraints. Precise predictions for the production and decay modes of the additional Higgs bosons are derived, and we present benchmark scenarios for searches at the LHC Run II. Flavor breaking at the electroweak scale as well as strong coupling effects demand a UV completion at the scale of a few $\mathrm{TeV}$, possibly within the reach of the LHC.

Keywords: Higgs Physics, Beyond Standard Model, Quark Masses and SM Parameters, Global Symmetries

ARXIV EPRINT: 1506.01719 


\section{Contents}

1 Introduction 1

2 Flavor from the electroweak scale $\quad 4$

3 Higgs couplings $\quad 6$

4 Higgs production and decay $\quad 9$

5 Constraints from flavor observables $\quad 14$

$\begin{array}{lll}5.1 & \text { Meson-antimeson mixing } & 14\end{array}$

$\begin{array}{lll}5.2 & \text { Flavor violating top decays } & 21\end{array}$

6 Perturbativity, unitarity, and electroweak precision measurements $\quad 22$

7 Collider searches for heavy extra scalars $\quad 25$

$\begin{array}{ll}7.1 \text { Couplings and total width of heavy scalars } & 25\end{array}$

$\begin{array}{ll}7.2 & \text { Analysis of production and decay channels }\end{array}$

8 Origin of the effective Yukawa couplings 34

9 Benchmark scenarios $\quad 36$

$\begin{array}{ll}10 \text { Conclusion } & 40\end{array}$

$\begin{array}{ll}\text { A The Higgs potential } & 43\end{array}$

B Box diagrams and loop functions $\quad 44$

$\begin{array}{ll}\text { C Random parameter generation and running } & 45\end{array}$

$\begin{array}{ll}\text { D Numerical input } & 46\end{array}$

$\begin{array}{ll}\text { E Branching ratios } & 48\end{array}$

\section{Introduction}

The origin of the observed hierarchies in fermion masses and mixings remains one of the most intricate puzzles of the Standard Model (SM) of particle physics. The sizes of the Yukawa couplings range over at least six orders of magnitude, and the magnitude of the CKM matrix elements varies between 1 and $10^{-3}$. Various extensions of the SM have been proposed in order to explain these hierarchies. In a seminal paper, Froggatt and Nielsen 

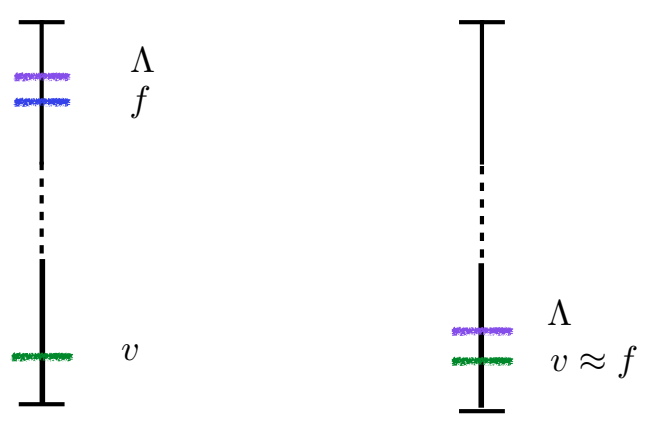

Figure 1. Mass scales in a generic Froggatt-Nielsen model (left) compared to the model proposed here (right).

introduced an abelian flavor symmetry by which only the top Yukawa coupling is allowed as a renormalizable operator [1]. The remaining Yukawa couplings are generated as higher order effective operators, schematically given by

$$
\mathcal{O}=y\left(\frac{S}{\Lambda}\right)^{n} \bar{Q} H q_{R}
$$

where lighter fermion masses require additional insertions of the Froggatt-Nielsen scalar, or flavon $S$. At a given energy scale, the flavon acquires a vacuum expectation value $\langle S\rangle=f$ and breaks the flavor symmetry. The fundamental Yukawa couplings $y$ are anarchic and hierarchies in the effective Yukawas are generated by the exponents $n$ of the ratio $f / \Lambda<1$, where $\Lambda$ is the scale at which new physics sets in. While the Froggatt-Nielsen paradigm does neither specify the flavor breaking scale $f$ nor the new physics scale $\Lambda$, the later implementation of this mechanism by Babu and Nandi [2] and Giudice and Lebedev [3] relate the flavor breaking scale to the electroweak scale. In particular, they propose $S / \Lambda \rightarrow H^{\dagger} H / \Lambda^{2}$ in (1.1). This interesting idea however has the shortcoming that the bilinear $H^{\dagger} H$ is a singlet under all symmetries, in particular it cannot carry a flavor charge. As a consequence, the number of flavon insertions needed in order to generate the observed fermion mass hierarchies is ad hoc and not related to a flavor symmetry. As briefly mentioned in [3], such a connection between the electroweak and the flavor breaking scale can however be motivated in a supersymmetric model featuring two Higgs doublets. Phenomenological constraints from the SM Higgs mass and signal strengths measurements exclude both the original Babu-Nandi-Giudice-Lebedev model as well as a possible (minimal) supersymmetric extension.

In this article, we propose a two Higgs doublet model, in which the two scalars $H_{u}$ and $H_{d}$ act jointly as the flavon field, such that $S / \Lambda \rightarrow H_{u} H_{d} / \Lambda^{2}$. As a consequence, the flavor breaking scale is set by the electroweak scale, $v \approx f$, and the new physics scale is in the ballpark of a few $\mathrm{TeV}$, as sketched in figure 1 .

In the present study we concentrate on the quark sector and include the tau Yukawa couplings, reserving a full treatment of the lepton sector for future work. We discuss Higgs phenomenology, as well as its connection to flavor physics and show the potential 
for distinctive discovery signals that point towards an explanation of flavor at the electroweak scale.

In our model, the Higgs dependent effective Yukawa couplings induce tree-level flavor changing neutral currents (FCNCs) mediated by the Higgs bosons. These FCNCs, although naively very large, turn out to be under control for a sizable region of the parameter space. To this end we perform a careful study of FCNC effects in $K-\bar{K}, B_{d, s}-\bar{B}_{d, s}$ mixing and estimate effects in the inclusive $B_{s} \rightarrow X_{s} \gamma$ decay as well as in the flavor-violating top decay $t \rightarrow h c$. Flavor diagonal couplings of the SM-like Higgs to quarks, as well as couplings between the Higgs and electroweak gauge bosons, are modified with respect to the SM. While the former are unique to our model, the latter are equivalent to the Higgs couplings to gauge bosons in generic two Higgs doublet models [4, 5]. This leads to deviations in both the Higgs production cross section and decay rates and we compute these effects for all relevant channels to compare them with current bounds from both the ATLAS and CMS experiments. We perform a global fit to all SM Higgs LHC data and we can accommodate the experimental data at a $2 \sigma$ level for a sizable range of model parameters. It is most remarkable, that the parameter space preferred by flavor observables has a significant overlap with the region preferred by the SM-like Higgs global fit.

A characteristic feature of this two Higgs doublet flavor model is, that both the constraints from Higgs signal strength measurements and flavor physics point to a parameter region far from the alignment/decoupling limit, such that the additional Higgs bosons cannot be arbitrarily heavy. Furthermore, electroweak precision observables favor a large mass splitting between charged and neutral scalars, while the neutral scalar masses are preferred to be almost degenerate. As a result, direct collider searches for the additional Higgs bosons are very powerful in probing this model. We analyze the LHC results from direct searches for the CP-even and CP-odd Higgs scalars as well as for the charged Higgs boson in various production and decay modes and identify the most promising channels for a discovery. Although the bosonic Higgs couplings parametrically correspond to the ones in a generic two Higgs doublet model, the parameter space singled out by flavor constraints and Higgs precision measurements leads to distinctive predictions for future searches at the LHC.

Altogether, the two Higgs doublet flavor model presented in this work provides an explanation for quark masses and mixing angles from physics at the electroweak scale, while providing new opportunities for Higgs phenomenology at the LHC. The model can be tested by high precision measurements of meson-antimeson mixing and implies a UV completion at a scale that can be probed at the LHC.

This paper is organized as follows: in section 2 we introduce our model, discuss the relevant parameters in the Yukawa sector and constraints from quark masses and mixing angles. We subsequently compute the Higgs couplings to quarks in section 3 . In section 4,5 and 6 we investigate constraints from Higgs, flavor and electroweak precision observables and map out the parameter space in agreement with these constraints. Section 7 contains a detailed analysis of present and future collider searches for the extra scalars. We comment on a possible UV completion section 8. In section 9 we present benchmarks for our model, before we summarize our main results in section 10 . 


\section{Flavor from the electroweak scale}

We consider a two Higgs doublet model in which fermion masses are generated by a Froggatt-Nielsen mechanism. We assume that the combination of the two scalar doublets $H_{u} H_{d}$ carries a non-zero flavor charge such that the flavon is replaced by

$$
\frac{S}{\Lambda} \rightarrow \frac{H_{u} H_{d}}{\Lambda^{2}} \equiv \frac{H_{u}^{T}\left(i \sigma_{2}\right) H_{d}}{\Lambda^{2}} .
$$

We assign opposite hypercharges to the two Higgs doublets and parametrize them as

$$
H_{u}=\frac{1}{\sqrt{2}}\left(\begin{array}{c}
v_{u}+\operatorname{Re} H_{u}^{0}+i \operatorname{Im} H_{u}^{0} \\
\sqrt{2} H_{u}^{-}
\end{array}\right), \quad H_{d}=\frac{1}{\sqrt{2}}\left(\begin{array}{c}
\sqrt{2} H_{d}^{+} \\
v_{d}+\operatorname{Re} H_{d}^{0}+i \operatorname{Im} H_{d}^{0}
\end{array}\right) .
$$

In this setup the electroweak scale sets the flavor breaking scale by

$$
\frac{f}{\Lambda} \rightarrow \frac{\left\langle H_{u} H_{d}\right\rangle}{\Lambda^{2}}=\frac{v_{u} v_{d}}{2 \Lambda^{2}}
$$

where

$$
v^{2}=v_{u}^{2}+v_{d}^{2}, \quad \frac{v_{u}}{v_{d}}=\tan \beta,
$$

with $v=246 \mathrm{GeV}$ and $0 \leq \beta \leq \pi / 2$, such that $v_{u}$ and $v_{d}$ are positive. We define the expansion parameter

$$
\varepsilon=\frac{v_{u} v_{d}}{2 \Lambda^{2}}=\frac{\tan \beta}{1+\tan \beta^{2}} \frac{v^{2}}{2 \Lambda^{2}} .
$$

We choose $\varepsilon=m_{b} / m_{t} \approx 1 / 60$, such that the Yukawa coupling for the bottom quarks corresponds to an effective operator with one insertion of the Higgs doublets $(n=1$ in terms of equation (1.1)). Therefore for $\tan \beta=1$, the new physics scale is approximately $\Lambda \approx 4 v \approx 1 \mathrm{TeV}$. If the fundamental Yukawa couplings in the UV completion are slightly larger than 1, this bound becomes weaker, and values of $\tan \beta>1$ are possible with a UV scale of the order of a TeV. Therefore, an ultraviolet completion at the TeV scale and $\tan \beta$ of $\mathcal{O}(1)$ are predictions of this model. We further discuss such a UV completion in section 8.

We consider the quarks and scalars in our model to be charged under a global $\mathrm{U}(1)_{F}$ symmetry. Therefore in the flavor eigenbasis the Yukawa sector of the SM is replaced by the effective Lagrangian (to leading order in powers of $\varepsilon$ )

$$
\mathcal{L}_{\text {Yuk }}=y_{i j}^{u}\left(\frac{H_{u} H_{d}}{\Lambda^{2}}\right)^{a_{i}-a_{u_{j}}-a_{H_{u}}} \bar{Q}_{i} H_{u} u_{R j}+y_{i j}^{d}\left(\frac{H_{u} H_{d}}{\Lambda^{2}}\right)^{a_{i}-a_{d_{j}}-a_{H_{d}}} \bar{Q}_{i} H_{d} d_{R j}+\text { h.c. },
$$

in which $a_{u_{j}}=a_{u}, a_{c}, a_{t}$, and $a_{d_{j}}=a_{d}, a_{s}, a_{b}$ denote the flavor charges of the three generations of up- and down-type quark singlets, $a_{i}=a_{1}, a_{2}, a_{3}$ the flavor charges of the three generations of quark doublets and $a_{H_{u}}, a_{H_{d}}$ the flavor charges of the Higgs doublets. The leading order Yukawa couplings in equation (2.6) reduce to the Yukawa sector of a two 
Higgs doublet model of type II in the limit of vanishing flavor charge $a_{i}, a_{u_{j}}, a_{d_{j}} \rightarrow 0$. Couplings of $H_{u}\left(H_{d}\right)$ to the down- (up-) type quarks are suppressed by additional powers of $\varepsilon .^{1}$ The fundamental Yukawa couplings $y_{i j}^{u}$ and $y_{i j}^{d}$ are considered to be anarchic and of $\mathcal{O}(1)$. In writing equation (2.6) we normalized the sum of the Higgs charges to $a_{H_{u}}+a_{H_{d}}=1$.

The effective Yukawa couplings are then given by

$$
\left(Y_{u}\right)_{i j}=y_{i j}^{u} \varepsilon^{a_{i}-a_{u_{j}}-a_{H_{u}}}, \quad\left(Y_{d}\right)_{i j}=y_{i j}^{d} \varepsilon^{a_{i}-a_{d_{j}}-a_{H_{d}}} .
$$

In (2.6) and (2.7), repeated indices between $y_{i j}$ and $\varepsilon^{a_{i}-a_{u_{j}}-a_{H_{u}}}$ are not summed over, i.e., for example $\left(Y_{u}\right)_{12}=y_{12}^{u} \varepsilon^{a_{1}-a_{c}-a_{H_{u}}}$. Thus the hierarchy of the effective Yukawa couplings is determined by the structure of the exponents of $\varepsilon$. The rotation to the mass eigenbasis is performed via

$$
Y_{u, d}=U_{u, d} \lambda_{u, d} W_{u, d}^{\dagger},
$$

with diagonal matrices given by

$$
\lambda_{u}=\frac{\sqrt{2}}{v_{u}} \operatorname{diag}\left(m_{u}, m_{c}, m_{t}\right), \quad \lambda_{d}=\frac{\sqrt{2}}{v_{d}} \operatorname{diag}\left(m_{d}, m_{s}, m_{b}\right),
$$

and unitary rotation matrices $U_{u, d}, W_{u, d}$.

In the following we fix the flavor charges of the quarks and Higgs bosons by imposing constraints from quark masses and the CKM matrix. If the charges of the three generations of quark doublets and singlets are ordered such that

$$
a_{1} \geq a_{2} \geq a_{3}, \quad a_{t} \geq a_{c} \geq a_{u}, \quad a_{b} \geq a_{s} \geq a_{d},
$$

one can derive the $\mathcal{O}(\varepsilon)$ dependence for the quark masses and rotation matrices [1],

$$
\begin{aligned}
& m_{u_{j}} \propto \frac{v_{u}}{\sqrt{2}} \varepsilon^{a_{j}-a_{u_{j}}-a_{H_{u}}}, \quad m_{d_{j}} \propto \frac{v_{d}}{\sqrt{2}} \varepsilon^{a_{j}-a_{d_{j}}-a_{H_{d}}} \\
& \left(U_{q}\right)_{i j} \propto \varepsilon^{\left|a_{i}-a_{j}\right|}, \quad\left(W_{u}\right)_{i j} \propto \varepsilon^{\left|a_{u_{i}}-a_{u_{j}}\right|}, \quad\left(W_{d}\right)_{i j} \propto \varepsilon^{\left|a_{d_{i}}-a_{d_{j}}\right|},
\end{aligned}
$$

for $i, j=1,2,3$. In the numerical analysis we will use the full unitary rotation matrices and include a scanning of anarchic Yukawa couplings with arbitrary phases and absolute values $\left|y_{i j}^{u, d}\right| \in[0.5,1.5]$. Six of the 11 flavor charges are fixed by the quark masses. We choose

$$
m_{t} \approx \frac{v_{u}}{\sqrt{2}}, \quad \frac{m_{b}}{m_{t}} \approx \frac{m_{c}}{m_{t}} \approx \varepsilon^{1}, \quad \frac{m_{s}}{m_{t}} \approx \varepsilon^{2}, \quad \frac{m_{d}}{m_{t}} \approx \frac{m_{u}}{m_{t}} \approx \varepsilon^{3}
$$

Additional conditions follow from the CKM matrix,

$$
V_{\mathrm{CKM}}=U_{u}^{\dagger} U_{d},
$$

\footnotetext{
${ }^{1}$ We also explored choices of flavor charges in which both up- and down-type quarks couple to one of the Higgs doublets at leading order (based on a two Higgs doublet model of type I), which will be discussed in a separate publication [6].
} 
by fixing

$$
\left(V_{\mathrm{CKM}}\right)_{12} \approx \varepsilon^{0}, \quad\left(V_{\mathrm{CKM}}\right)_{13} \approx\left(V_{\mathrm{CKM}}\right)_{23} \approx \varepsilon^{1} .
$$

These conditions end up fixing only two parameters. Including the normalization of the Higgs charges $a_{H_{u}}+a_{H_{d}}=1$ and our choice of $a_{H_{u}}=1$, we have 10 conditions on 11 parameters. $^{2}$ The remaining choice allows for an overall shift of quark flavor charges. Physical quantities however only depend on invariant differences. Thus the remaining choice does not have any phenomenological consequences and we set

$$
\begin{array}{llll}
a_{H_{u}}=1, & a_{1}=2, & a_{u}=-2, & a_{d}=-1, \\
a_{H_{d}}=0, & a_{2}=2, & a_{c}=0, & a_{s}=0, \\
& a_{3}=1, & a_{t}=0, & a_{b}=0 .
\end{array}
$$

If the last condition (2.14) is replaced by

$$
\left(V_{\mathrm{CKM}}\right)_{12} \approx\left(V_{\mathrm{CKM}}\right)_{13} \approx\left(V_{\mathrm{CKM}}\right)_{23} \approx \varepsilon^{0},
$$

only the structure of the quark masses is explained by the flavor charges, while the hierarchical form of the CKM matrix is determined by the fundamental Yukawas $y_{i j}^{u}, y_{i j}^{d}$. In this case, a suitable choice of flavor charges read

$$
\begin{array}{llll}
a_{H_{u}}=1, & a_{1}=2, & a_{u}=-2, & a_{d}=-1, \\
a_{H_{d}}=0, & a_{2}=2, & a_{c}=0, & a_{s}=0, \\
& a_{3}=2, & a_{t}=1, & a_{b}=1 .
\end{array}
$$

This choice of charges is motivated by considerably weaker constraints from flavor observables due to the aligned charges for the left-handed quark fields.

A detailed implementation of lepton masses and mixing angles is beyond the scope of this work. We will however define the couplings of the tau leptons to the scalars in our model, since they are important for the Higgs phenomenology. We set

$$
\mathcal{O}_{\tau}^{b}=y_{\tau} \frac{H_{u} H_{d}}{\Lambda^{2}} \bar{\tau}_{L} H_{d} \tau_{R}
$$

such that $m_{\tau} / m_{t} \approx \varepsilon$.

\section{Higgs couplings}

The Yukawa interactions give rise to modifications to flavor diagonal Higgs couplings as well as potentially dangerous flavor changing neutral currents. In the flavor eigenbasis the interaction between quarks and the real neutral components of the Higgs doublet scalars follows from (2.6) and we obtain

$$
\begin{aligned}
\mathcal{L}_{0}= & \left(Y_{u}\right)_{i j}\left[\left(1+a_{i}-a_{u_{j}}-a_{H_{u}}\right) \operatorname{Re} H_{u}^{0}+\left(a_{i}-a_{u_{j}}-a_{H_{u}}\right) \tan \beta \operatorname{Re} H_{d}^{0}\right] \bar{u}_{L_{i}} u_{R_{j}} \\
& +\left(Y_{d}\right)_{i j}\left[\left(1+a_{i}-a_{d_{j}}-a_{H_{d}}\right) \operatorname{Re} H_{d}^{0}+\left(a_{i}-a_{d_{j}}-a_{H_{d}}\right) \cot \beta \operatorname{Re} H_{u}^{0}\right] \bar{d}_{L_{i}} d_{R_{j}}+\text { h.c.. }
\end{aligned}
$$

\footnotetext{
${ }^{2}$ Different choices for the normalization condition or the Higgs charges, e.g. $a_{H_{d}}=1, a_{H_{u}}=0$, do not change the physics of this model but will only imply different assignments for the quark flavor charges.
} 
We rotate to the quark mass eigenbasis, according to equation (2.8) and introduce the Higgs mass eigenstates as defined in appendix A. The rotation of the scalars gives rise to the following couplings between the scalar mass eigenstates and quarks

$$
\mathcal{L}_{0}=\left(b_{u}\right)_{i j} \bar{u}_{L_{i}} h u_{R_{j}}+\left(b_{d}\right)_{i j} \bar{d}_{L_{i}} h d_{R_{j}}+\left(B_{u}\right)_{i j} \bar{u}_{L_{i}} H u_{R_{j}}+\left(B_{d}\right)_{i j} \bar{d}_{L_{i}} H d_{R_{j}}+\text { h.c. },
$$

in which

$$
\begin{aligned}
\left(b_{u}\right)_{i j} & =\left(Y_{u}\right)_{i j}\left[\left(1+a_{i}-a_{u_{j}}-a_{H_{u}}\right) \cos \alpha-\left(a_{i}-a_{u_{j}}-a_{H_{u}}\right) \sin \alpha \tan \beta\right], \\
\left(b_{d}\right)_{i j} & =\left(Y_{d}\right)_{i j}\left[-\left(1+a_{i}-a_{d_{j}}-a_{H_{d}}\right) \sin \alpha+\left(a_{i}-a_{d_{j}}-a_{H_{d}}\right) \cos \alpha \cot \beta\right], \\
\left(B_{u}\right)_{i j} & =\left(Y_{u}\right)_{i j}\left[\left(1+a_{i}-a_{u_{j}}-a_{H_{u}}\right) \sin \alpha+\left(a_{i}-a_{u_{j}}-a_{H_{u}}\right) \cos \alpha \tan \beta\right], \\
\left(B_{d}\right)_{i j} & =\left(Y_{d}\right)_{i j}\left[\left(1+a_{i}-a_{d_{j}}-a_{H_{d}}\right) \cos \alpha+\left(a_{i}-a_{d_{j}}-a_{H_{d}}\right) \sin \alpha \cot \beta\right] .
\end{aligned}
$$

After rotating to the quark mass eigenbasis,

$$
\begin{aligned}
g_{h u_{i} u_{j}} & =\left(U_{u}^{\dagger}\right)_{i k}\left(b_{u}\right)_{k l}\left(W_{u}\right)_{l j}, & & g_{H u_{i} u_{j}}=\left(U_{u}^{\dagger}\right)_{i k}\left(B_{u}\right)_{k l}\left(W_{u}\right)_{l j}, \\
g_{h d_{i} d_{j}} & =\left(U_{d}^{\dagger}\right)_{i k}\left(b_{d}\right)_{k l}\left(W_{d}\right)_{l j}, & & g_{H d_{i} d_{j}}=\left(U_{d}^{\dagger}\right)_{i k}\left(B_{d}\right)_{k l}\left(W_{d}\right)_{l j},
\end{aligned}
$$

we find for the couplings of the light neutral scalar $h$,

$$
\begin{aligned}
g_{h u_{i} u_{j}} & =\left(\frac{m_{u}}{v}\right)_{i j} \delta_{i j}\left[\frac{c_{\alpha}}{s_{\beta}}-a_{H_{u}} f(\alpha, \beta)\right]+f(\alpha, \beta)\left[\mathcal{Q}_{i j}^{u}\left(\frac{m_{u}}{v}\right)_{j j}-\left(\frac{m_{u}}{v}\right)_{i i} \mathcal{U}_{i j}\right], \\
g_{h d_{i} d_{j}} & =\left(\frac{m_{d}}{v}\right)_{i j} \delta_{i j}\left[-\frac{s_{\alpha}}{c_{\beta}}-a_{H_{d}} f(\alpha, \beta)\right]+f(\alpha, \beta)\left[\mathcal{Q}_{i j}^{d}\left(\frac{m_{d}}{v}\right)_{j j}-\left(\frac{m_{d}}{v}\right)_{i i} \mathcal{D}_{i j}\right],
\end{aligned}
$$

and for the heavy neutral scalar $H$,

$$
\begin{aligned}
g_{H u_{i} u_{j}} & =\left(\frac{m_{u}}{v}\right)_{i j} \delta_{i j}\left[\frac{s_{\alpha}}{s_{\beta}}-a_{H_{u}} F(\alpha, \beta)\right]+F(\alpha, \beta)\left[\mathcal{Q}_{i j}^{u}\left(\frac{m_{u}}{v}\right)_{j j}-\left(\frac{m_{u}}{v}\right)_{i i} \mathcal{U}_{i j}\right], \\
g_{H d_{i} d_{j}} & =\left(\frac{m_{d}}{v}\right)_{i j} \delta_{i j}\left[\frac{c_{\alpha}}{c_{\beta}}-a_{H_{d}} F(\alpha, \beta)\right]+F(\alpha, \beta)\left[\mathcal{Q}_{i j}^{d}\left(\frac{m_{d}}{v}\right)_{j j}-\left(\frac{m_{d}}{v}\right)_{i i} \mathcal{D}_{i j}\right],
\end{aligned}
$$

in which $m_{u}=\operatorname{diag}\left(m_{u}, m_{c}, m_{t}\right), m_{d}=\operatorname{diag}\left(m_{d}, m_{s}, m_{b}\right)$ and we define $s_{\varphi}=\sin \varphi, c_{\varphi}=$ $\cos \varphi$ and $t_{\varphi}=\tan \varphi$, for any angle $\varphi$. In both (3.5) and (3.6), repeated indices are not summed over and we suppress the chirality index of the fermions $q_{i} \equiv q_{L_{i}}, q_{j} \equiv q_{R_{j}}$. We make use of the following trigonometric functions

$$
\begin{gathered}
f(\alpha, \beta)=\frac{c_{\alpha}}{s_{\beta}}-\frac{s_{\alpha}}{c_{\beta}}=c_{\beta-\alpha}\left(\frac{1}{t_{\beta}}-t_{\beta}\right)+2 s_{\beta-\alpha}, \\
F(\alpha, \beta)=\frac{c_{\alpha}}{c_{\beta}}+\frac{s_{\alpha}}{s_{\beta}}=2 c_{\beta-\alpha}+s_{\beta-\alpha}\left(t_{\beta}-\frac{1}{t_{\beta}}\right),
\end{gathered}
$$

which are universal for up- and down-type quarks. We also define the matrices

$$
\begin{aligned}
\mathcal{Q}_{i j}^{u} & =\sum_{\ell=1}^{3}\left(U_{u}\right)_{\ell i}^{*}\left(U_{u}\right)_{\ell j} a_{\ell}, & \mathcal{Q}_{i j}^{d} & =\sum_{\ell=1}^{3}\left(U_{d}\right)_{\ell i}^{*}\left(U_{d}\right)_{\ell j} a_{\ell}, \\
\mathcal{U}_{i j} & =\sum_{k=1}^{3}\left(W_{u}\right)_{k i}^{*}\left(W_{u}\right)_{k j} a_{u_{k}}, & \mathcal{D}_{i j} & =\sum_{k=1}^{3}\left(W_{d}\right)_{k i}^{*}\left(W_{d}\right)_{k j} a_{d_{k}} .
\end{aligned}
$$


The structure of these matrices is fixed by the flavor charges, as given at the end of section 2 . We find for the flavor charges in (2.15),

$$
\mathcal{Q}^{u} \sim \mathcal{Q}^{d} \sim\left(\begin{array}{ccc}
2 & \varepsilon^{2} & \varepsilon \\
\varepsilon^{2} & 2 & \varepsilon \\
\varepsilon & \varepsilon & 1
\end{array}\right), \quad \mathcal{U} \sim\left(\begin{array}{ccc}
-2 & \varepsilon^{2} & \varepsilon^{2} \\
\varepsilon^{2} & \varepsilon^{2} & \varepsilon^{4} \\
\varepsilon^{2} & \varepsilon^{4} & \varepsilon^{4}
\end{array}\right), \quad \mathcal{D} \sim\left(\begin{array}{ccc}
-1 & \varepsilon & \varepsilon \\
\varepsilon & \varepsilon^{2} & \varepsilon^{2} \\
\varepsilon & \varepsilon^{2} & \varepsilon^{2}
\end{array}\right)
$$

For completeness, we also give the expressions for these matrices in the case of the flavor charges (2.17),

$$
\mathcal{Q}^{u} \sim \mathcal{Q}^{d} \sim\left(\begin{array}{lll}
2 & 0 & 0 \\
0 & 2 & 0 \\
0 & 0 & 2
\end{array}\right), \quad \mathcal{U} \sim\left(\begin{array}{ccc}
-2 & \varepsilon^{2} & \varepsilon^{3} \\
\varepsilon^{2} & \varepsilon^{2} & \varepsilon \\
\varepsilon^{3} & \varepsilon & 1
\end{array}\right), \quad \mathcal{D} \sim\left(\begin{array}{ccc}
-1 & \varepsilon & \varepsilon^{2} \\
\varepsilon & \varepsilon^{2} & \varepsilon \\
\varepsilon^{2} & \varepsilon & 1
\end{array}\right) .
$$

Note that all flavor off-diagonal Higgs couplings are proportional to these matrices. In the limit of degenerate flavor charges $a_{i}, a_{u_{i}}$ or $a_{d_{i}}$, these matrices become diagonal and do not induce any flavor violating couplings. For the flavor charges (2.17), therefore only $\mathcal{U}$ and $\mathcal{D}$ generate FCNCs.

In addition, all flavor violating couplings of the scalars in (3.5) and (3.6) are proportional to the trigonometric functions in (3.7). In the limit $f(\alpha, \beta)=0$, all flavor off-diagonal couplings of the light Higgs vanish and the diagonal couplings are independent of both $c_{\beta-\alpha}$ and $t_{\beta}$, and approach their SM values (up to a sign). It should be noted that this sign difference corresponds to the wrong-sign Yukawa coupling in a generic two Higgs doublet model $[7,8]$. We will come back to these observations when we discuss flavor observables in section 5. The limit $c_{\beta-\alpha}=0$, associated with decoupling [9-11] or alignment $[5,10-12]$ is not the SM, but corresponds to the model proposed by Babu, Nandi [2], and Giudice and Lebedev [3].

The pseudoscalar mass eigenstate $A$ is obtained through the rotation (A.3) and its couplings to quark mass eigenstates can be derived from (3.6), by replacing

$$
g_{A q_{i} q_{j}}=\left.i g_{H q_{i} q_{j}}\right|_{c_{\alpha} \rightarrow s_{\beta}, s_{\alpha} \rightarrow c_{\beta}} .
$$

Finally, the charged Higgs couplings can also be obtained from (2.6) and are independent of the flavor charges. After rotation to quark and Higgs mass eigenstates, see (A.4), we obtain

$$
\mathcal{L}_{ \pm}=\frac{\sqrt{2}}{v} \frac{1}{t_{\beta}}\left(m_{u}\right)_{k j}\left(V_{\mathrm{CKM}}^{\dagger}\right)_{i k} \bar{d}_{L_{i}} H^{-} u_{R_{j}}+\frac{\sqrt{2}}{v} t_{\beta}\left(m_{d}\right)_{k j}\left(V_{\mathrm{CKM}}\right)_{i k} \bar{u}_{L_{i}} H^{+} d_{R_{j}}+\text { h.c. } .
$$

The couplings of the charged Higgs to quarks are therefore equivalent to the ones in the two Higgs doublet model of type II, see for example [13]. 


\section{Higgs production and decay}

A light SM-like Higgs has been discovered at the LHC in various decay channels. While observations are mainly in the ballpark of SM expectations, there is still room for new physics. The modified flavor diagonal fermion couplings of the light Higgs $h$ introduced in the previous section as well as modified gauge boson couplings lead to deviations in both production cross section and decay rates. In the following we compute these deviations and compare the results with the proton-proton collision data at $\sqrt{s}=7$ and $8 \mathrm{TeV}$ obtained from the ATLAS [14] and CMS [15] experiments.

For a given Higgs boson production channel and decay rate into specific final states $X$, normalized to the SM values, we define the signal strength parameter

$$
\mu_{X}=\frac{\sigma_{\text {prod }}}{\sigma_{\text {prod }}^{\text {SM }}} \frac{\Gamma_{h \rightarrow X}}{\Gamma_{h \rightarrow X}^{\text {SM }}} \frac{\Gamma_{h, \text { tot }}^{\text {SM }}}{\Gamma_{h}} .
$$

New physics can enter each of these three quantities: the production cross section $\sigma_{\text {prod }}$, the partial decay rate $\Gamma_{h \rightarrow X}$ and the total width $\Gamma_{h \text {,tot }}$. We quantify the changes in flavor diagonal couplings of the Higgs to fermions $f=t, b, \tau$ and to vector bosons $V=W^{ \pm}, Z$ with respect to the SM by

$$
\begin{gathered}
g_{h f f}=\kappa_{f} g_{h f f}^{\mathrm{SM}}=\kappa_{f} \frac{m_{f}}{v}, \\
g_{h V V}=\kappa_{V} g_{h V V}^{\mathrm{SM}}=\kappa_{V} \frac{2 m_{V}^{2}}{v},
\end{gathered}
$$

such that $\kappa_{f}=\kappa_{V}=1$ in the SM limit.

It follows from equation (3.5), that the coupling of the light Higgs to the top quark is rescaled by

$$
\kappa_{t}=\frac{c_{\alpha}}{s_{\beta}}=\frac{c_{\beta-\alpha}}{t_{\beta}}+s_{\beta-\alpha} .
$$

As a result, these couplings are modified in the same way as in two Higgs doublet models of type II, see for example $[5,13,16]$. However, couplings to the other flavors significantly differ from the couplings in generic two Higgs doublet models because of the Higgs dependent effective Yukawas, such that the Higgs-bottom coupling is rescaled by

$$
\kappa_{b}=-2 \frac{s_{\alpha}}{c_{\beta}}+\frac{c_{\alpha}}{s_{\beta}}=3 s_{\beta-\alpha}+c_{\beta-\alpha}\left(\frac{1}{t_{\beta}}-2 t_{\beta}\right) .
$$

Note, that for $f(\alpha, \beta)=0$, any dependence on $c_{\beta-\alpha}$ and $t_{\beta}$ cancels in (4.3) and (4.4) and we find that $\kappa_{t}=1$ and $\kappa_{b}=-1$ and therefore the light Higgs has couplings to fermions of SM strength. We illustrate the parameter dependence of the square of these couplings in figure 2. In the right panel of figure 2 the value of $\kappa_{b}^{2}$ goes through zero signalizing $\kappa_{b}$ changes sign and becomes negative in the upper right (lower left) corner for $\cos (\beta-\alpha)>0$ $(\cos (\beta-\alpha)<0)$. The structure of these couplings has significant impact on the Higgs boson production cross sections and decay rates. Further, the coupling of the light Higgs boson to charm quarks is rescaled by

$$
\kappa_{c}=3 s_{\beta-\alpha}+c_{\beta-\alpha}\left(\frac{2}{t_{\beta}}-t_{\beta}\right) .
$$



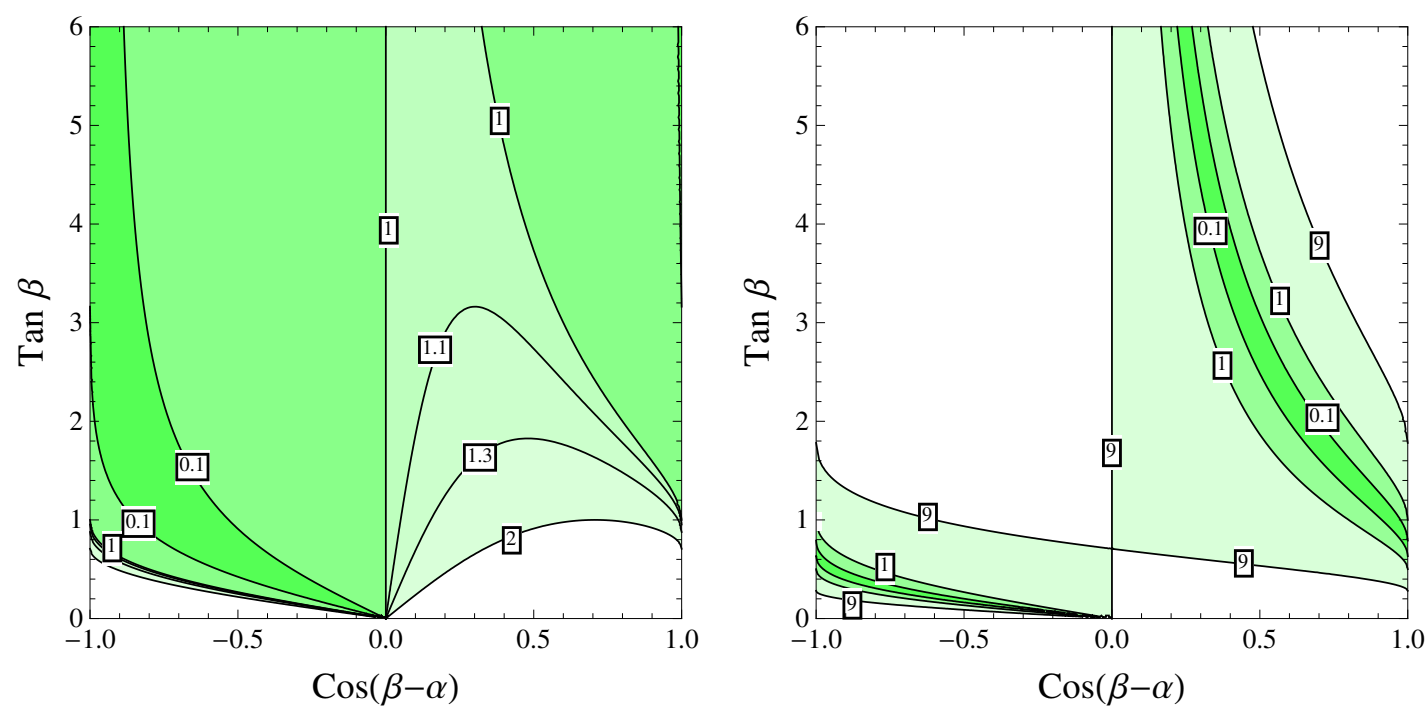

Figure 2. Contours of $\kappa_{t}^{2}$ (left) and $\kappa_{b}^{2}$ (right) in the $\cos (\beta-\alpha)-\tan \beta$ plane. $\kappa_{t}^{2}=\kappa_{b}^{2}=1$ corresponds to the SM limit, for $\kappa_{b}$ up to a sign in the right upper (lower left) corner for $\cos (\beta-\alpha)>$ $0(\cos (\beta-\alpha)<0)$. The decoupling/alignment limit corresponds to the Babu-Nandi-GiudiceLebedev model.

In general, fermion mixing effects generate corrections to the couplings, since the flavor charges of the quarks are not universal. These effects are encoded in the matrices $\mathcal{Q}^{u, d}, \mathcal{U}$ and $\mathcal{D}$ given in equation (3.8). For flavor-diagonal Higgs couplings to fermions we neglect corrections of $\mathcal{O}(\varepsilon)$. For couplings of the light Higgs boson to tau leptons we assume that a mechanism similar to our findings in the quark sector is responsible for generating masses, such that

$$
\kappa_{\tau}=\kappa_{b} .
$$

For the couplings of the light Higgs to vector bosons we obtain

$$
\kappa_{V}=s_{\beta-\alpha},
$$

which is the same as in generic two Higgs doublet models.

The gluon fusion initiated Higgs production, neglecting light quark contributions in the fermion loops, is defined normalized to the SM value as

$$
\frac{\sigma_{g g \rightarrow h}}{\sigma_{g g \rightarrow h}^{\mathrm{SM}}}=\kappa_{t}^{2}\left|1+\xi_{b} \frac{\kappa_{b}}{\kappa_{t}}\right|^{2},
$$

where $\xi_{b}=-0.032+0.035 i$ depends on the loop functions given in [4]. Therefore for values of $\kappa_{b}$ of $\mathcal{O}(1)$, the main Higgs production channel is to leading order indistinguishable from a type II two Higgs doublet model. Vector Boson Fusion (VBF) and Higgsstrahlung (VH) are both rescaled by $\kappa_{V}$, while associated Higgs boson production with a top pair is modified by $\kappa_{t}$,

$$
\frac{\sigma_{t \bar{t} \rightarrow h}}{\sigma_{t \bar{t} \rightarrow h}^{\mathrm{SM}}}=\kappa_{t}^{2} \quad \text { and } \quad \frac{\sigma_{\mathrm{VBF}}}{\sigma_{\mathrm{VBF}}^{\mathrm{SM}}}=\frac{\sigma_{\mathrm{VH}}}{\sigma_{\mathrm{VH}}^{\mathrm{SM}}}=\kappa_{V}^{2}
$$


Therefore the three production processes rescale with the same factors as in generic two Higgs doublet models, as given e.g. in $[5,13,16]$.

The partial decay widths of the light Higgs into SM fermions $f$ and gauge bosons $V=W^{ \pm}, Z$ can similarly be written as

$$
\frac{\Gamma_{h \rightarrow f f}}{\Gamma_{h \rightarrow f f}^{\mathrm{SM}}}=\kappa_{f}^{2}, \quad \text { and } \quad \frac{\Gamma_{h \rightarrow V V}}{\Gamma_{h \rightarrow V V}^{\mathrm{SM}}}=\kappa_{V}^{2} .
$$

Both top quark and $W^{ \pm}$boson loops enter the diphoton decay width [29],

$$
\frac{\Gamma_{h \rightarrow \gamma \gamma}}{\Gamma_{h \rightarrow \gamma \gamma}^{\mathrm{SM}}}=\left|0.28 \kappa_{t}-1.28 \kappa_{W}+\delta\right|^{2},
$$

in which contributions from light fermions are neglected and contributions from charged scalar loops are encoded in $\delta$. We find for $M_{H^{ \pm}} \gtrsim 300 \mathrm{GeV}$ a contribution of less than $\delta \lesssim 0.04$ and set it to zero in the following $[9,29]$.

Expressed in terms of the rescaling factors $\kappa_{t}, \kappa_{b}, \kappa_{c}, \kappa_{\tau}$ and $\kappa_{V}$, the total Higgs boson width is given by $[30,31]$

$$
\frac{\Gamma_{h}}{\Gamma_{h}^{S M}} \approx 0.57 \kappa_{b}^{2}+0.25 \kappa_{V}^{2}+0.09 \kappa_{t}^{2}+0.06 \kappa_{\tau}^{2}+0.026 \kappa_{c}^{2}+0.004,
$$

where $\Gamma_{h}^{\mathrm{SM}}=4.07 \mathrm{MeV}$ [32] and we assume $h \rightarrow Z \gamma$ and even rarer modes to be SM-like. These contributions are collected in the constant term 0.004 .

The partial decay width into bottom quarks has a very different dependence on $\tan \beta$ and $\cos (\beta-\alpha)$ than in the generic type II two Higgs doublet model. This plays a relevant role in defining the allowed region in parameter space, since the bottom quark partial decay width dominates the total decay width, that in turn importantly affects the signal strength for all channels.

In figure 3 we show the result of a global $\chi^{2}$ fit based on the data collected in table 1. Symmetrized errors are used for the fit. The left panel shows the plot for ATLAS and the right panel the plot for CMS. The two fit parameters are $c_{\beta-\alpha}$ and $t_{\beta}$. The $1 \sigma$ and $2 \sigma$ regions consistent with the LHC data are shaded in dark and light red, respectively. It is clear, that the preferred parameter space is different from generic two Higgs doublet models, for which regions close to the alignment or decoupling limit $c_{\beta-\alpha}=0$ are favorable. $[5,11,33]$. In our case, $c_{\beta-\alpha}=0$ corresponds to the Babu-Nandi-Giudice-Lebedev model [2,3], which is clearly disfavored by the data. We observe, that while the allowed region for ATLAS is slightly smaller than in the case of CMS, both fits show a preference for values of $c_{\beta-\alpha}>0$ and $t_{\beta} \gtrsim 1$. The more constrained region of parameter space for ATLAS can be understood by the larger central values of $\mu_{Z}, \mu_{W}$ and $\mu_{\gamma}$ in the dominant gluon fusion channel, that are less compatible with larger values of $\kappa_{b}$, see figure 2 . The white area between the two branches in both fits can be explained by very small values of $\kappa_{b}$ for which all other branching fractions grow. Overall, the fermion couplings prefer a region in parameter space, where they approach their SM values, with the caveat that the value of the bottom Higgs coupling $\kappa_{b}$ has a negative sign with respect to the SM value in the upper right branch of 

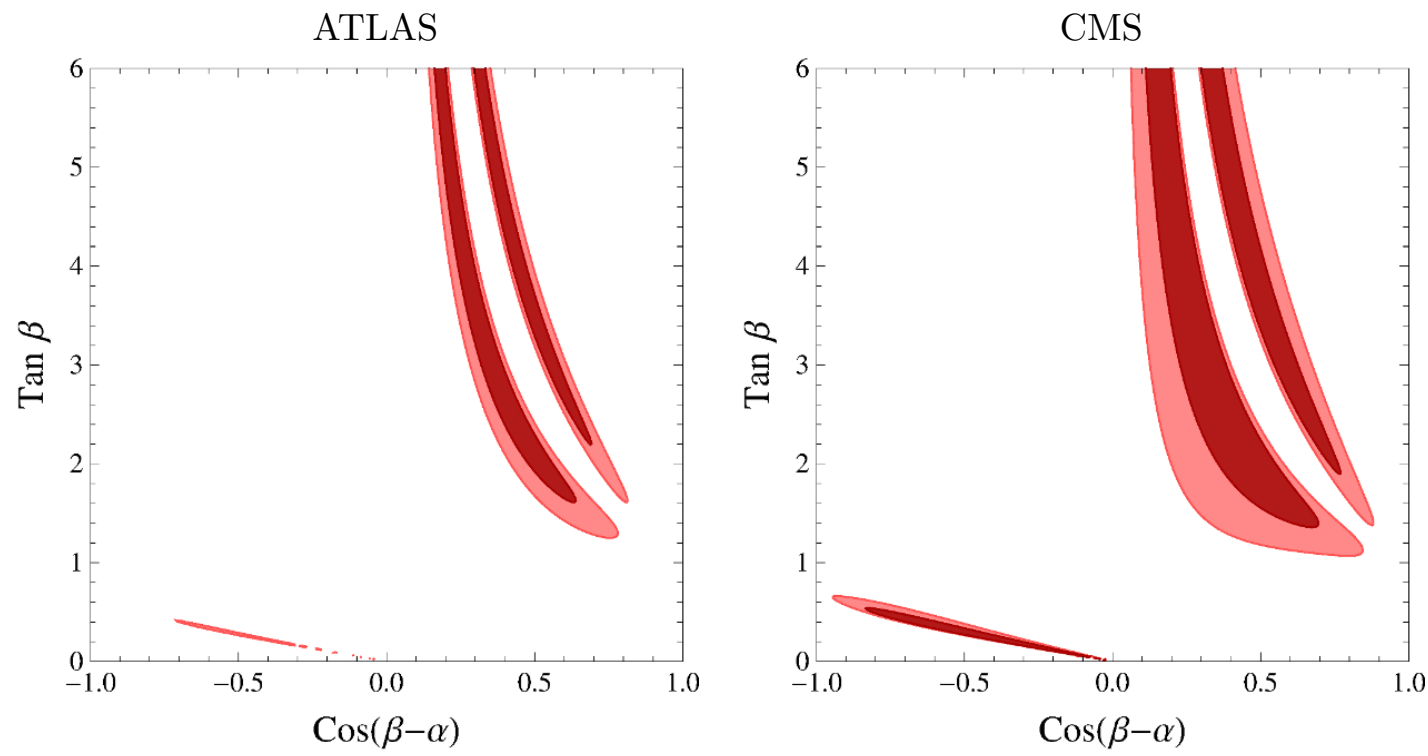

Figure 3. Allowed $1 \sigma$ (dark red) and $2 \sigma$ (light red) regions, for a global fit to ATLAS and CMS data from measurements of Higgs boson decays in the left and right panel, respectively. The channels entering the fit are summarized in table 1 and errors are symmetrized.

\begin{tabular}{|c|c|c|c|}
\hline Decay Mode & $\begin{array}{l}\text { Production Channels } \\
\sigma_{g g \rightarrow h}, \sigma_{t \bar{t} \rightarrow h}\end{array}$ & $\begin{array}{l}\text { Production Channels } \\
\sigma_{V B F}, \sigma_{V H}\end{array}$ & Experiment \\
\hline$h \rightarrow W W^{*}$ & $\begin{array}{l}\mu_{W}=1.02_{-0.26}^{+0.29}[17] \\
\mu_{W} \simeq 0.75 \pm 0.35[18]\end{array}$ & $\begin{array}{l}\mu_{W}=1.27_{-0.45}^{+0.53}[17] \\
\mu_{W} \simeq 0.7 \pm 0.85[18]\end{array}$ & $\begin{array}{l}\text { ATLAS } \\
\text { CMS }\end{array}$ \\
\hline$h \rightarrow Z Z^{*}$ & $\begin{array}{l}\mu_{Z}=1.7_{-0.4}^{+0.5}[19] \\
\mu_{Z}=0.8_{-0.36}^{+0.46}[20]\end{array}$ & $\begin{array}{l}\mu_{Z}=0.3_{-0.9}^{+1.6}[19] \\
\mu_{Z}=1.7_{-2.1}^{+2.2}[20]\end{array}$ & $\begin{array}{l}\text { ATLAS } \\
\text { CMS }\end{array}$ \\
\hline$h \rightarrow \gamma \gamma$ & $\begin{aligned} \mu_{\gamma} & =1.32 \pm 0.38[21] \\
\mu_{\gamma} & =1.13_{-0.31}^{+0.37}[22]\end{aligned}$ & $\begin{array}{l}\mu_{\gamma}=0.8 \pm 0.7[21] \\
\mu_{\gamma}=1.16_{-0.58}^{+0.63}[22]\end{array}$ & $\begin{array}{l}\text { ATLAS } \\
\text { CMS }\end{array}$ \\
\hline$h \rightarrow \bar{b} b$ & $\begin{aligned} \mu_{b} & =1.5 \pm 1.1[23] \\
\mu_{b} & =0.67_{-1.33}^{+1.35}[25]\end{aligned}$ & $\begin{array}{l}\mu_{b}=0.52 \pm 0.32 \pm 0.24[24] \\
\mu_{b}=1.0 \pm 0.5[26]\end{array}$ & $\begin{array}{l}\text { ATLAS } \\
\text { CMS }\end{array}$ \\
\hline$h \rightarrow \tau \tau$ & $\begin{array}{l}\mu_{\tau}=2.0 \pm 0.8_{-0.8}^{+1.2} \pm 0.3[27] \\
\mu_{\tau} \simeq 0.5_{-0.7}^{+0.8}[28]\end{array}$ & $\begin{array}{l}\mu_{\tau}=1.24_{-0.45}^{+0.49+0.29} \pm 0.08[27] \\
\mu_{\tau} \simeq 1.1_{-0.5}^{+0.7}[28]\end{array}$ & $\begin{array}{l}\text { ATLAS } \\
\text { CMS }\end{array}$ \\
\hline
\end{tabular}

Table 1. Input data for the global $\chi^{2}$-fit of Higgs production and decay with references. The data includes all updated results of the pp collision data at $\sqrt{s}=7$ and $8 \mathrm{TeV}$ obtained from the ATLAS [14] and CMS [15] experiments. 

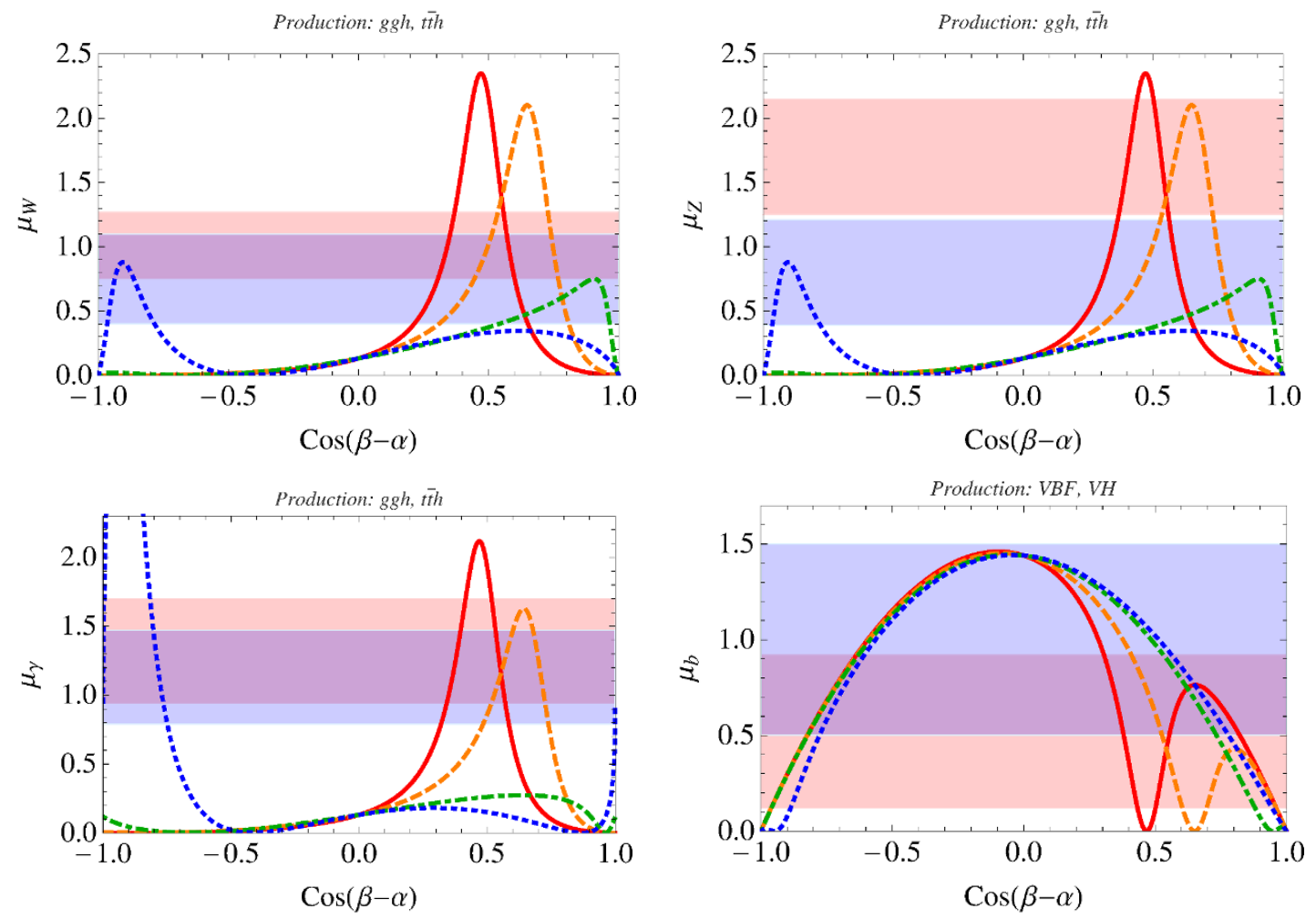

Figure 4. The upper panels show the signal strengths $\mu_{W}$ (left) and $\mu_{Z}$ (right) and the lower panel the signal strengths $\mu_{\gamma}$ (left) and $\mu_{b}$ (right) plotted against $c_{\beta-\alpha}$. The red (blue) band is the symmetrized $1 \sigma$ region of the corresponding ATLAS (CMS) measurement. Each plot shows curves for $t_{\beta}=3$ (solid red), $t_{\beta}=2$ (dashed orange), $t_{\beta}=1$ (dot-dashed green) and $t_{\beta}=0.5$ (dotted blue).

the allowed red region. Note also that small values of $c_{\beta-\alpha}$ correspond to larger $t_{\beta}$ in the region preferred by the global fit as follows from equation (4.4).

In order to understand the features of the global fit, we present the signal strengths of the relevant decay channels in figure 4 . In these plots, the red (blue) band is the $1 \sigma$ region of the corresponding ATLAS (CMS) measurement. Each plot shows the prediction of a particular signal strength for $\mu_{W}, \mu_{Z}, \mu_{\gamma}$ and $\mu_{b}$, depending on $c_{\beta-\alpha}$ for $t_{\beta}=3$ (solid red), $t_{\beta}=2$ (dashed orange), $t_{\beta}=1$ (dot-dashed green) and $t_{\beta}=0.5$ (dotted blue). Excluding all but these four observables only marginally changes the global fits. For $t_{\beta} \gtrsim 1$ all four measurements prefer values of $c_{\beta-\alpha}>0$. There is also an allowed region for $c_{\beta-\alpha}<0$ for values of $t_{\beta}<1$, however as will be shown later this region is phenomenologically less interesting.

We conclude, that the global fit to LHC Higgs measurements accommodates tan $\beta$ of $\mathcal{O}(1)$ for sizable values of $\cos (\beta-\alpha)$ away from the decoupling/alignment limit. This is a nontrivial result, given that $\tan \beta$ is already constrained to be of order one from the bound on the new physics scale. As we discuss below, values of $\tan \beta \lesssim 5$ are in agreement with flavor constraints as well as a possible UV completion scale in the $\mathrm{TeV}$ to a few $\mathrm{TeV}$ range. 

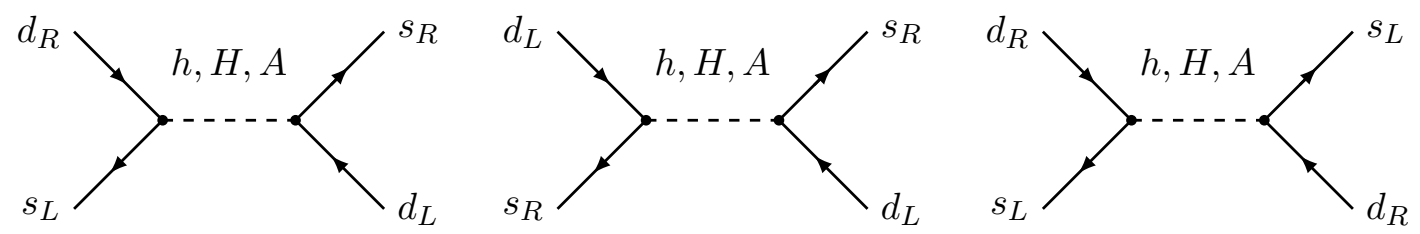

Figure 5. Tree-level contributions to $\Delta S=2$ processes.

\section{Constraints from flavor observables}

In addition to modifications of flavor-diagonal couplings, the misalignment of the mass and coupling matrices induces flavor changing couplings of the light Higgs $h$, the heavy neutral scalar $H$ and the pseudoscalar $A$. These couplings generate FCNCs at tree-level, which are subject to strong constraints from neutral meson oscillations. In the following we calculate and analyze contributions to the relevant observables. We further estimate effects in $b \rightarrow s \gamma$ and give the prediction for the flavor-violating top decay $t \rightarrow h c$.

\subsection{Meson-antimeson mixing}

In the $K-\bar{K}$ system, contributions from Higgs mediated FCNCs are captured by the effective Hamiltonian

$$
\begin{aligned}
\mathcal{H}_{\mathrm{NP}}^{\Delta S=2}= & C_{1}^{s d}\left(\bar{s}_{L} \gamma_{\mu} d_{L}\right)^{2}+\tilde{C}_{1}^{s d}\left(\bar{s}_{R} \gamma_{\mu} d_{R}\right)^{2}+C_{2}^{s d}\left(\bar{s}_{R} d_{L}\right)^{2}+\tilde{C}_{2}^{s d}\left(\bar{s}_{L} d_{R}\right)^{2} \\
& +C_{4}^{s d}\left(\bar{s}_{R} d_{L}\right)\left(\bar{s}_{L} d_{R}\right)+C_{5}^{s d}\left(\bar{s}_{L} \gamma_{\mu} d_{L}\right)\left(\bar{s}_{R} \gamma^{\mu} d_{R}\right)+h . c .
\end{aligned}
$$

At tree-level, the corresponding Wilson coefficients can be read off from the diagrams in figure 5 [34],

$$
\begin{aligned}
C_{2}^{s d} & =-\frac{\left(g_{h d s}^{*}\right)^{2}}{m_{h}^{2}}-\frac{\left(g_{H d s}^{*}\right)^{2}}{M_{H}^{2}}-\frac{\left(g_{A d s}^{*}\right)^{2}}{M_{A}^{2}}, \\
\tilde{C}_{2}^{s d} & =-\frac{g_{h s d}^{2}}{m_{h}^{2}}-\frac{g_{H s d}^{2}}{M_{H}^{2}}-\frac{g_{A s d}^{2}}{M_{A}^{2}}, \\
C_{4}^{s d} & =-\frac{g_{h s d} g_{h d s}^{*}}{2 m_{h}^{2}}-\frac{g_{H s d} g_{H d s}^{*}}{2 M_{H}^{2}}-\frac{g_{A s d} g_{A d s}^{*}}{2 M_{A}^{2}} .
\end{aligned}
$$

Similar expressions hold for $B_{s}-\bar{B}_{s}$ mixing, with $s d \rightarrow b s, B_{d}-\bar{B}_{d}$ mixing, with $s d \rightarrow$ $b d$ and $D-\bar{D}$ mixing with $s d \rightarrow u c$. Contributions from Higgs boson exchange are only suppressed by the weak scale, but the Froggatt-Nielsen mechanism induces an additional suppression of flavor off-diagonal couplings by the masses of the involved quarks as well as the expansion parameter $\varepsilon$. The relative size of the Wilson coefficients (5.2) depends therefore strongly on the explicit flavor structure. For the flavor charge assignment (2.15), which is tailored to explain quark masses as well as CKM mixing angles, we collect the results in the left hand side of table 2. 


\begin{tabular}{|c|ccc|ccc|}
\hline & \multicolumn{3}{|c|}{ Scenario (2.15) } & \multicolumn{3}{c|}{ Scenario (2.17) } \\
\hline$\Delta F=2$ & $c_{2}^{i j}$ & $\tilde{c}_{2}^{i j}$ & $c_{4}^{i j}$ & $c_{2}^{i j}$ & $\tilde{c}_{2}^{i j}$ & $c_{4}^{i j}$ \\
\hline$s d$ & $\varepsilon^{4} m_{s}^{2}$ & $\varepsilon^{2} m_{s}^{2}$ & $\varepsilon^{3} m_{s}^{2}$ & $\varepsilon^{2} m_{d}^{2}$ & $\varepsilon^{2} m_{s}^{2}$ & $\varepsilon^{2} m_{d} m_{s}$ \\
$b d$ & $\varepsilon^{2} m_{b}^{2}$ & $\varepsilon^{2} m_{b}^{2}$ & $\varepsilon^{2} m_{b}^{2}$ & $\varepsilon^{4} m_{d}^{2}$ & $\varepsilon^{4} m_{b}^{2}$ & $\varepsilon^{4} m_{d} m_{b}$ \\
$b s$ & $\varepsilon^{2} m_{b}^{2}$ & $\varepsilon^{4} m_{b}^{2}$ & $\varepsilon^{3} m_{b}^{2}$ & $\varepsilon^{2} m_{s}^{2}$ & $\varepsilon^{2} m_{b}^{2}$ & $\varepsilon^{2} m_{s} m_{b}$ \\
$u c$ & $\varepsilon^{4} m_{c}^{2}$ & $\varepsilon^{4} m_{c}^{2}$ & $\varepsilon^{4} m_{c}^{2}$ & $\varepsilon^{4} m_{c}^{2}$ & $\varepsilon^{4} m_{u}^{2}$ & $\varepsilon^{4} m_{u} m_{c}$ \\
\hline
\end{tabular}

Table 2. Flavor specific part of the Wilson coefficients for meson-antimeson mixing in the case of the flavor charge assignments (2.15) with flavor structure (3.9) (left) and flavor charge assignments (2.17) with flavor structure (3.10) (right).

In the case of $K-\bar{K}$ mixing, we find that the largest coefficient is $\tilde{C}_{2}$ with

$$
\begin{aligned}
\tilde{C}_{2}^{s d} & =-\frac{\tilde{c}_{2}^{s d}}{v^{2}}\left\{\frac{f(\alpha, \beta)^{2}}{m_{h}^{2}}+\frac{F(\alpha, \beta)^{2}}{M_{H}^{2}}-\left(t_{\beta}+\frac{1}{t_{\beta}}\right)^{2} \frac{1}{M_{A}^{2}}\right\} \\
& \approx \frac{-10^{-15}}{\mathrm{GeV}^{2}}\left\{f(\alpha, \beta)^{2}+F(\alpha, \beta)^{2} \frac{m_{h}^{2}}{M_{H}^{2}}-\left(t_{\beta}+\frac{1}{t_{\beta}}\right)^{2} \frac{m_{h}^{2}}{M_{A}^{2}}\right\},
\end{aligned}
$$

where we factored out the light Higgs mass in the second line, the trigonometric functions $f(\alpha, \beta)$ and $F(\alpha, \beta)$ are defined in (3.7), and $\tilde{c}_{2}^{s d}$ is the flavor-dependent part of the Wilson coefficient given in table 2 . The same expression holds for the Wilson coefficient $C_{2}^{s d}$, with the additional $\varepsilon^{2}$ suppression due to the replacement of $\tilde{c}_{2}^{s d} \rightarrow c_{2}^{s d}$. The flavor-dependent Wilson coefficient $c_{4}^{s d}$ is also suppressed by $\varepsilon$ with respect to $\tilde{c}_{2}^{s d}$, but the minus sign in the last line of (5.3) is replaced by a plus, which corresponds to a constructive interference of the different contributions,

$$
\begin{aligned}
C_{4}^{s d} & =-\frac{c_{4}^{s d}}{v^{2}}\left\{\frac{f(\alpha, \beta)^{2}}{m_{h}^{2}}+\frac{F(\alpha, \beta)^{2}}{M_{H}^{2}}+\left(t_{\beta}+\frac{1}{t_{\beta}}\right)^{2} \frac{1}{M_{A}^{2}}\right\} \\
& \approx \frac{-1.7 \times 10^{-17}}{\mathrm{GeV}^{2}}\left\{f(\alpha, \beta)^{2}+F(\alpha, \beta)^{2} \frac{m_{h}^{2}}{M_{H}^{2}}+\left(t_{\beta}+\frac{1}{t_{\beta}}\right)^{2} \frac{m_{h}^{2}}{M_{A}^{2}}\right\} .
\end{aligned}
$$

The limit of exact cancellation in $C_{2}^{s d}$ and $\tilde{C}_{2}^{s d}$ and maximal interference in $C_{4}^{s d}$ corresponds to the $\mathrm{SU}(2)_{L}$ symmetric limit, in which operators of the type $\left(\bar{s}_{L} d_{R}\right)^{2}$ are forbidden [35]. In table 3, we present the current bounds on the Wilson coefficients at the electroweak scale for the different meson systems, based on [36]. These bounds have been derived by assuming that new physics only contributes to a single Wilson coefficient and can therefore only be taken as a rough upper limit. For $K-\bar{K}$ mixing, the strongest constraint comes from the CP violating observable $\epsilon_{K}$, such that the bounds on the imaginary part of the Wilson coefficient is cited. Since we assume arbitrary phases, the estimate (5.3) holds for both real and imaginary parts of the Wilson coefficients. Comparing (5.3) with the bound in table 3 shows that a partial cancellation in $\tilde{C}_{2}^{s d}$ is necessary in order to comply with the limit. For $M_{A}, M_{H}>m_{h}$, this corresponds to a preferred region in the $\cos (\beta-\alpha)-\tan \beta$ plane. In the left panel of figure 7 we show the preferred region, 


\begin{tabular}{|c|cccc|}
\hline$i$ & 1 & 2 & 4 & 5 \\
\hline $\operatorname{Im} C_{i}^{s d}$ & $\lesssim 2 \times 10^{-15}$ & $\lesssim 1 \times 10^{-16}$ & $\lesssim 7 \times 10^{-17}$ & $\lesssim 9 \times 10^{-16}$ \\
$\operatorname{Im} C_{i}^{u c}$ & $\lesssim 2 \times 10^{-14}$ & $\lesssim 2 \times 10^{-14}$ & $\lesssim 1 \times 10^{-14}$ & $\lesssim 1 \times 10^{-13}$ \\
$\left|C_{i}^{b d}\right|$ & $\lesssim 1 \times 10^{-12}$ & $\lesssim 4 \times 10^{-13}$ & $\lesssim 6 \times 10^{-13}$ & $\lesssim 1 \times 10^{-12}$ \\
$\left|C_{i}^{b s}\right|$ & $\lesssim 1 \times 10^{-11}$ & $\lesssim 2 \times 10^{-12}$ & $\lesssim 4 \times 10^{-12}$ & $\lesssim 6 \times 10^{-12}$ \\
\hline
\end{tabular}

Table 3. Model-independent bounds on Wilson coefficients for meson-antimeson mixing evaluated at the electroweak scale in units of $\mathrm{GeV}^{-2}$ [36], taking into account the running described in appendix D. The same bounds hold for the Wilson coefficients with flipped chirality $C_{i} \rightarrow \tilde{C}_{i}$.

for which $\left|\tilde{C}_{2}^{s d}\right|<10^{-16} / \mathrm{GeV}^{2}$ (shaded orange), assuming $M_{A}=M_{H}=500 \mathrm{GeV}$. Contributions to $C_{4}^{s d}$ can be enhanced by the constructive interference between the scalar contributions. Also, the bound on $C_{4}^{s d}$ is particularly strong, because it is enhanced from Renormalization Group ( $\mathrm{RG}$ ) running as well as from the matrix element, that scales like $M_{K}^{2} /\left(m_{s}+m_{d}\right)^{2} \approx 14$, see appendix $\mathrm{C}$ for details. However, the additional suppression shown in table 2 gives $C_{4}^{s d}=\varepsilon \tilde{C}_{2}^{s d}$, such that a slight enhancement from interference effects is allowed. In the left panel of figure 7 we show the region in the $\cos (\beta-\alpha)-\tan \beta$ plane for which $\left|C_{4}^{s d}\right|<7 \times 10^{-17} / \mathrm{GeV}^{2}$ (shaded blue).

In addition to tree-level exchanges, various one-loop contributions can potentially become large. The relevant diagrams are shown in figure 6 . The contributions from the box diagrams of type (a) are completely analogous to the ones in a type II two Higgs doublet model, because the couplings of the charged Higgs (3.12) are indistinguishable between the two models. The leading contribution enters $C_{1}^{s d}$ and comes from the box with one charged Higgs, a $W^{ \pm}$boson and top quarks running in the loop and one finds [37]

$$
C_{1, \text { box }}^{s d} \propto \frac{1}{16 \pi^{2}} \frac{1}{t_{\beta}^{2}}\left(\frac{m_{t}^{2}}{v^{2}} V_{t s}^{*} V_{t d}\right)^{2} \frac{1}{M_{H^{+}}^{2}} \approx 9 \times 10^{-16}\left(\frac{500 \mathrm{GeV}}{M_{H^{+}}}\right)^{2} \mathrm{GeV}^{-2},
$$

where in the last equality we set $t_{\beta}=1$. For $t_{\beta} \lesssim 1$, this contribution is of the order of the largest tree-level contribution. We therefore require $t_{\beta} \gtrsim 1$ in order to be in compliance with experimental bounds in table 3 . In principle, there are also contributions from box diagrams to the other operators in (5.1) as well as box diagrams with neutral scalar exchange, but both are chirally suppressed by powers of light quark masses over the electroweak scale and turn out to be negligible. The loop diagrams labeled $(b)$ and $(c)$ in figure 6 are also suppressed. Diagrams of type $(b)$ have the same coupling structure as the tree-level diagrams, but are additionally suppressed by a loop factor. Diagrams of type (c) are enhanced with respect to (5.4) by the light Higgs couplings to the top quark or charged scalars, but suppressed by CKM elements and a loop factor, such that we find for $C_{4}^{s d}[38]$

$$
\frac{C_{4, \text { penguin }}^{s d}}{C_{4, h}^{s d}} \approx \frac{1}{16 \pi^{2}} \frac{m_{t}^{2}}{v^{2}} \frac{V_{t s}^{*} V_{t d}}{\varepsilon^{2} f(\alpha, \beta)} \approx 10^{-3} .
$$

The equivalent diagram with a charm quark in the loop is of the same order. 

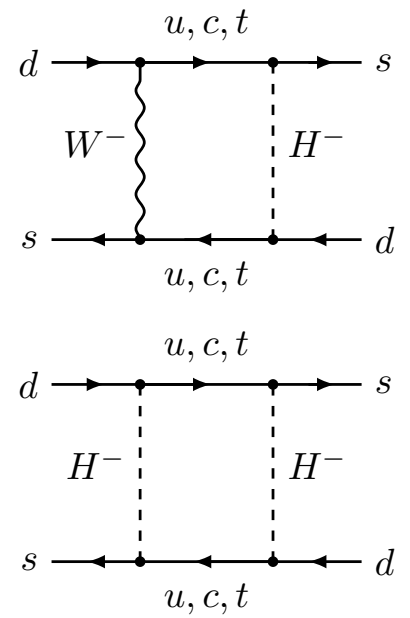

(a)
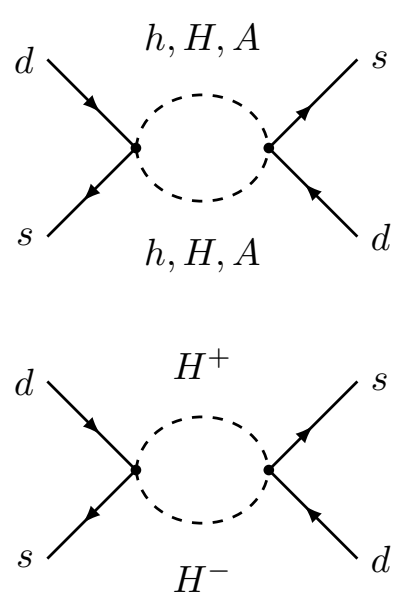

(b)
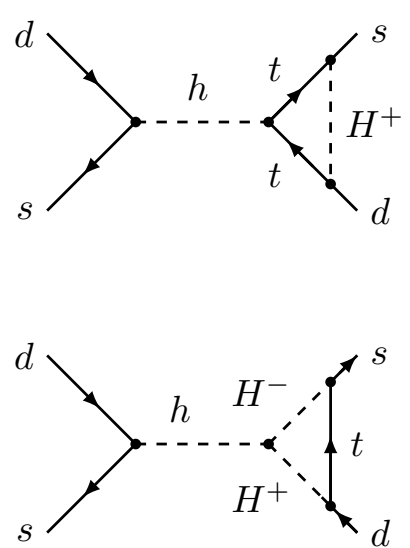

$(c)$

Figure 6. Three types of one loop contributions to $\Delta S=2$ processes.

Having considered all different contributions we will map out the parameter space in the $\cos (\beta-\alpha)-\tan \beta$ plane in which the prediction for $\epsilon_{K}$ in our model agrees with the experimental bound within $2 \sigma$ in a numerical analysis. For this purpose we define

$$
C_{\epsilon_{K}}=\frac{\operatorname{Im}\left\langle K^{0}\left|\mathcal{H}_{\mathrm{full}}^{\Delta S}\right|=2 \mid \bar{K}^{0}\right\rangle}{\operatorname{Im}\left\langle K^{0}\left|\mathcal{H}_{\mathrm{SM}}^{\Delta S}\right| \bar{K}^{0}\right\rangle},
$$

where $\mathcal{H}_{\text {full }}^{\Delta S=2}=\mathcal{H}_{\mathrm{SM}}^{\Delta S=2}+\mathcal{H}_{\mathrm{NP}}^{\Delta S=2}$ includes the Standard Model contribution. We compute the Wilson coefficients at the scale of the light Higgs and for $M_{H}=M_{A}=M_{H^{ \pm}}=500 \mathrm{GeV}$ respectively, using the full expressions for the Wilson coefficients including tree-level and leading box diagrams. We collect the full analytic expressions of the latter in appendix B. In the next step, the Wilson coefficients in (5.1) are evolved down from the mass scale of the scalars to the scale $\mu=2 \mathrm{GeV}$ at which the hadronic matrix elements are evaluated using the RG equations in [39]. The hadronic matrix elements are taken from [40] and collected with the other numerical input in appendix D. We randomly generate a sample set of points of fundamental Yukawa couplings, defined in (2.6), with $\left|y_{i j}^{u, d}\right| \in[0.5,1.5]$ and with arbitrary phases. We require the SM quark masses and Wolfenstein parameters to be reproduced within two standard deviations. More details to the procedure and input parameters can be found in appendices $\mathrm{C}$ and $\mathrm{D}$. At this stage, the mixing angles $\alpha$ and $\beta$ from the Higgs sector still remain free parameters and our sample set only fixes the fundamental Yukawas.

In the right panel of figure 7 we show the percentage of sample points which reproduce $C_{\epsilon_{K}}^{\exp }$ within $2 \sigma$ in the $\cos (\beta-\alpha)-\tan \beta$ plane. We employ the value extracted from a fit to the CKM triangle by the UTfit group [41],

$$
C_{\epsilon_{K}}^{\exp }=1.05_{-0.28}^{+0.36} @ 95 \% \mathrm{CL} .
$$



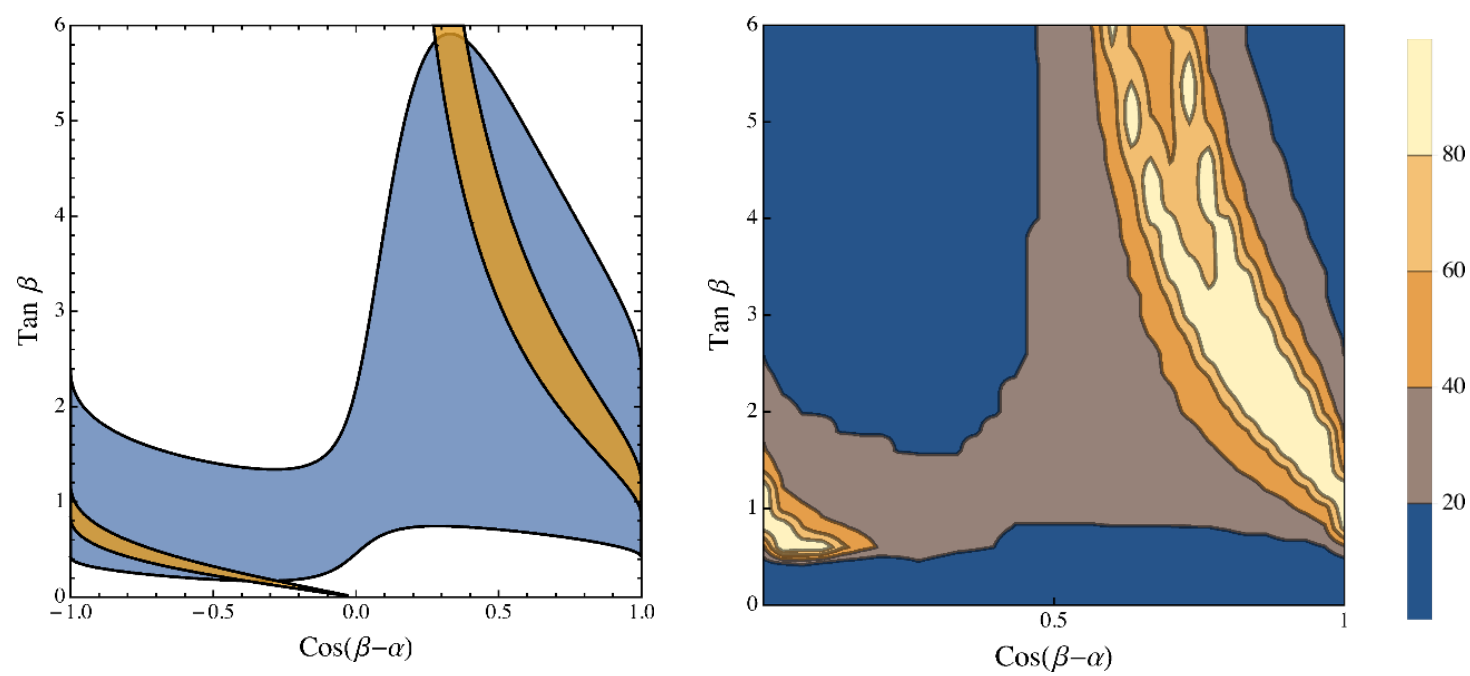

Figure 7. The left panel shows the region in the $c_{\beta-\alpha}-t_{\beta}$ plane for which the tree-level contributions to $\left|\tilde{C}_{2}^{s d}\right| \leq 10^{-16} / \mathrm{GeV}^{2}$ (orange) and the tree-level contributions to $\left|C_{4}^{s d}\right| \leq 7 \times 10^{-17} / \mathrm{GeV}^{2}$ (blue). In the right panel we show regions of parameter space in which our sample points reproduce $C_{\varepsilon_{K}}$ within two standard deviations. The color coding indicates the percentage of points in agreement with the experimental constraint. In both plots, the scalar masses are $M_{A}=M_{H}=M_{H^{+}}=500 \mathrm{GeV}$.

The result shows good agreement with the estimate of the separate contributions shown in the left panel of figure 7 . The area for which $t_{\beta}<0.5$ is cut off, because of the one-loop contributions from charged Higgs exchange [42]. We find a large region of parameter space for which our model prediction is in agreement with the experimental bound without any tuning of parameters.

In the case of $B_{d}-\bar{B}_{d}$ and $B_{s}-\bar{B}_{s}$ mixing, the effective Lagrangian, as well as the tree-level contributions to the Wilson coefficients from scalar and pseudoscalar exchange can be read off from (5.1) and (5.2) with the replacements $s \leftrightarrow b$ and $d \leftrightarrow d, s$, respectively. The angle dependence of the Wilson coefficients is universal and therefore only the flavor dependent part changes from (5.3) and (5.4), such that the parametric dependence presented in the left panel of figure 7 also holds in the $B$ sector. For the Wilson coefficients it follows from table 2 ,

$$
\begin{aligned}
& C_{4}^{b d} \approx C_{2}^{b d} \approx \tilde{C}_{2}^{b d} \approx C_{2}^{b s} \propto \frac{m_{b}^{2}}{v^{2}} \frac{\varepsilon^{2}}{m_{h}^{2}} \approx \frac{2.5 \times 10^{-12}}{\mathrm{GeV}^{2}}, \\
& \tilde{C}_{2}^{b s} \propto \frac{m_{b}^{2}}{v^{2}} \frac{\varepsilon^{4}}{m_{h}^{2}} \approx \frac{7 \times 10^{-16}}{\mathrm{GeV}^{2}}, \quad C_{4}^{b s} \propto \frac{m_{b}^{2}}{v^{2}} \frac{\varepsilon^{3}}{m_{h}^{2}} \approx \frac{4 \times 10^{-14}}{\mathrm{GeV}^{2}} .
\end{aligned}
$$

The corresponding bounds in table 3 imply, that $C_{2}^{b s}$ is at the border of the naive bound, while a much larger contribution to $C_{4}^{b s}$ is allowed. The contributions to $C_{4}^{b d}, C_{2}^{b d}$ and $\tilde{C}_{2}^{b d}$ are too large almost in the entire $\cos (\beta-\alpha)-\tan \beta$ plane, and therefore demand cancellations implying important restrictions for the permitted region of our parameter space. 

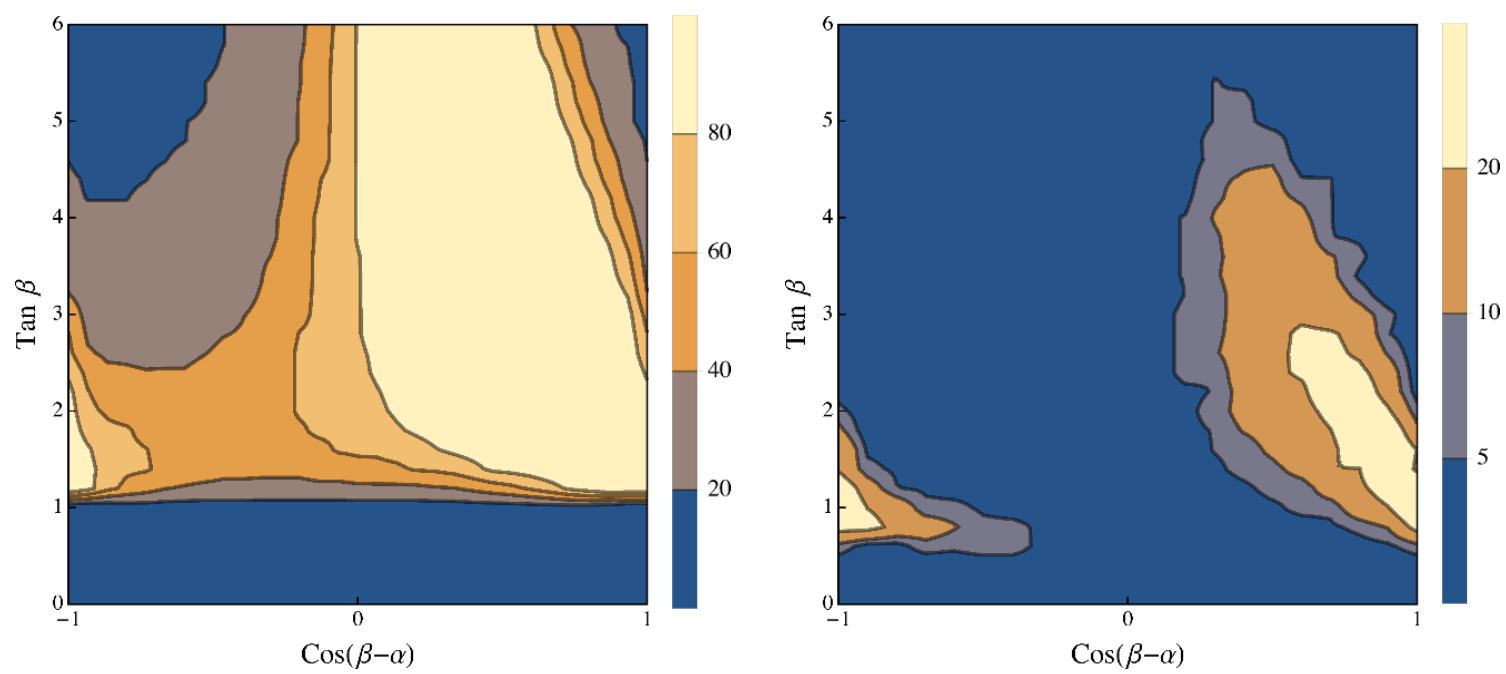

Figure 8. In the left (right) panel we show regions of parameter space in which our sample points reproduce $C_{B_{s}}\left(C_{B_{d}}\right)$ within two standard deviations. The color coding indicates the percentage of points in agreement with the experimental constraint. In both plots, the scalar masses are $M_{A}=M_{H}=M_{H^{+}}=500 \mathrm{GeV}$.

At the one-loop level, box diagrams generate the contributions

$$
C_{1, \text { box }}^{b q} \propto \frac{1}{16 \pi^{2}} \frac{1}{t_{\beta}^{2}}\left(\frac{m_{t}^{2}}{v^{2}} V_{t b}^{*} V_{t q}\right)^{2} \frac{1}{M_{H^{+}}^{2}} \approx\left(\frac{500 \mathrm{GeV}}{M_{H^{+}}}\right)^{2} \begin{cases}5 \times 10^{-13} \mathrm{GeV}^{-2}, & q=d, \\ 1 \times 10^{-11} \mathrm{GeV}^{-2}, & q=s,\end{cases}
$$

for $\tan \beta=1$. In the $B_{s}-\bar{B}_{s}$ system for low $\tan \beta$, this contribution becomes larger than all tree-level contributions. Since the box is only sensitive to charged Higgs couplings, we expect comparable constraints as in a two Higgs doublet model of type II. In addition, since the contribution is independent of $\cos (\beta-\alpha)$, we expect a universal lower bound on $\tan \beta$, as observed in the left panel of figure 8 . For both the $B_{d, s}-\bar{B}_{d, s}$ system we also include the box diagram contributions to the other Wilson coefficients, which are suppressed by $m_{b} / m_{W}$. The corresponding expressions are collected in appendix B.

Analogous to (5.7), we define

$$
C_{B_{q}} e^{2 i \phi_{B_{q}}}=\frac{\left\langle B_{q}^{0}\left|\mathcal{H}_{\mathrm{full}}^{\Delta B=2}\right| \bar{B}_{q}^{0}\right\rangle}{\left\langle B_{q}^{0}\left|\mathcal{H}_{\mathrm{SM}}^{\Delta B=2}\right| \bar{B}_{q}^{0}\right\rangle}
$$

such that $C_{B_{q}}=\Delta m_{q} / \Delta m_{q}^{\mathrm{SM}}$ measures new physics effects in the mass difference and new phases enter $\phi_{B_{q}}$. In the left (right) panel of figure 8, we present the percentage of sample points in agreement with the experimental constraints at $95 \% \mathrm{CL}$ for $C_{B_{s}}^{\exp }\left(C_{B_{d}}^{\exp }\right)$, based on the results obtained from the UTfit group [41],

$$
C_{B_{s}}^{\exp }=1.052_{-0.152}^{+0.178} @ 95 \% \mathrm{CL}, \quad C_{B_{d}}^{\exp }=1.07_{-0.31}^{+0.36} @ 95 \% \mathrm{CL} .
$$

In both plots we choose $M_{H}=M_{A}=M_{H^{+}}=500 \mathrm{GeV}$. As expected from our estimate above, in the $B_{s}-\bar{B}_{s}$ system, we find good agreement with the experimental bounds for a 


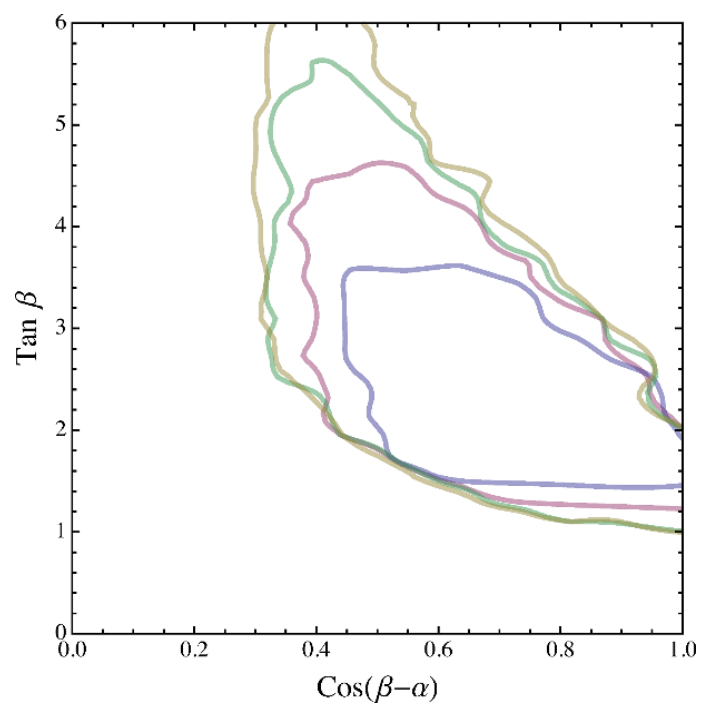

Figure 9. Boundaries of the regions in which $10 \%$ of our parameter points agree with the $C_{B_{d}}$ at the $95 \% \mathrm{CL}$ in the positive $c_{\beta-\alpha}$ plane. The different colors correspond to $M \equiv M_{A}=M_{H}=M_{H^{+}}=$ $400 \mathrm{GeV}$ (blue) $M=500 \mathrm{GeV}$ (purple), $M=600 \mathrm{GeV}$ (green), and $M=700 \mathrm{GeV}$ (light brown).

large region of parameter space. For the $B_{d}-\bar{B}_{d}$ system, we find only a small fraction of the parameter space in agreement with the experimental constraints. Since the new physics effects in all Wilson coefficients are too large, accidental cancellations in the fundamental Yukawa couplings are in effect in order to achieve agreement with data. As a consequence, slightly tuned Yukawa couplings as well as rather heavy extra scalars $M_{A} \approx M_{H} \approx 500 \mathrm{GeV}$ are necessary in order to agree with the bounds from $B_{d}-\bar{B}_{d}$ mixing. In the following, we will adopt the $10 \%$ contour as the fine-tuning bound from flavor observables on the parameter space. Figure 9 shows the corresponding contours in the positive $c_{\beta-\alpha}$ plane for $M \equiv M_{A}=M_{H}=M_{H^{+}}=400 \mathrm{GeV}$ (blue), $M=500 \mathrm{GeV}$ (purple), $M=600 \mathrm{GeV}$ (green), and $M=700 \mathrm{GeV}$ (light brown). The bound for low $\tan \beta$ comes from the charged Higgs loops in $B_{s}-\bar{B}_{s}$ mixing. A future, more precise measurement of meson-antimeson mixing can reveal deviations from the SM prediction or further constrain the allowed parameter space, if no new physics effect is found.

In $D-\bar{D}$ mixing, all tree-level contributions to the Wilson coefficients are strongly suppressed,

$$
C_{4}^{u c} \approx C_{2}^{u c} \approx \tilde{C}_{2}^{u c} \propto \frac{m_{c}^{2}}{v^{2}} \frac{\varepsilon^{4}}{m_{h}^{2}} \approx \frac{3.4 \times 10^{-17}}{\mathrm{GeV}^{2}}
$$

In contrast to the down-sector however, the box diagram with neutral Higgs exchange is not suppressed by light quark masses, because the dominant contribution comes from the top in the loop [43]. The leading box contributions of the light Higgs to the coefficient $C_{1}^{u c}$ can therefore be larger than all tree-level effects

$$
C_{1}^{u c} \approx-\frac{1}{128 \pi^{2}}\left(\frac{m_{t}}{v} \varepsilon f(\alpha, \beta)\right)^{4} D_{2}\left(m_{t}, m_{h}\right)
$$




$$
\begin{aligned}
& =-\frac{1}{128 \pi^{2}}\left(\frac{m_{t}}{v} \varepsilon f(\alpha, \beta)\right)^{4} \frac{m_{h}^{4}-m_{t}^{4}-2 m_{h}^{2} m_{t}^{2} \log \left(\frac{m_{t}^{2}}{m_{h}^{2}}\right)}{\left(m_{h}^{2}-m_{t}^{2}\right)^{3}} \\
& \approx-\frac{2 \times 10^{-16}}{\mathrm{GeV}^{2}}
\end{aligned}
$$

for $f(\alpha, \beta)=1$, and the loop function defined in appendix B. Boxes with heavy Higgs insertions are further suppressed. However, the corresponding bound in table 3 is orders of magnitude weaker than our estimate. The $D-\bar{D}$ system will therefore not induce further constraints.

In all the above analyses, we have concentrated on the solution for the flavor charges (2.15), but the situation is quite different for the flavor charges given in (2.17). From (3.10) it follows, that the contributions to the Wilson coefficients are highly suppressed, as is explicit in the flavor-dependent parts of the Wilson coefficients given on the right hand side of table 2. This shows, that although constraints from the $B_{s}-\bar{B}_{s}$ and $K-\bar{K}$ systems remain the same, the constraints from the $B_{d}-\bar{B}_{d}$ system can be very much relaxed due to the different charge assignment. Therefore, if only the hierarchies in the quark masses are explained by a Froggatt-Nielsen mechanism at the weak scale, but the CKM mixing angles have a different origin, bounds from meson-antimeson mixing are very mild and do not lead to any severe restrictions on the parameter space.

Rare Kaon and $B_{d, s}$ decays can in principle be subject to large corrections, but depend crucially on the implementation of the lepton sector, which will be discussed elsewhere. Processes in which the neutral scalars only enter at loop-level, such as $\operatorname{Br}\left(B_{s} \rightarrow X_{s} \gamma\right)$ are generically dominated by charged Higgs contributions, which are larger than the contributions from the neutral Higgs by a factor of

$$
\frac{m_{t} V_{t b} V_{t s}^{*}}{m_{b} f(\alpha, \beta) \varepsilon} \approx \mathcal{O}\left(10^{2}-10^{3}\right),
$$

for $f(\alpha, \beta)=0.1-1$. We will therefore adopt the bounds from $\operatorname{Br}\left(B_{s} \rightarrow X_{s} \gamma\right)$ on the charged scalar mass in two Higgs doublet models for $\tan \beta \gtrsim 2$, considering values within a $3 \sigma$ band in order to account for uncertainties of higher order corrections not included in the theoretical computation [44, 45],

$$
M_{H^{ \pm}} \gtrsim 358(480) \mathrm{GeV} @ 99 \%(95 \%) \mathrm{CL} .
$$

\subsection{Flavor violating top decays}

We consider the flavor violating decays of the top quark $t \rightarrow h c$ and $t \rightarrow h u$. In contrast to the SM, in which flavor violating top quark decays are loop suppressed, in our model the top quark has tree-level couplings to the light Higgs and other up-type flavors. The corresponding branching ratios $\operatorname{Br}(t \rightarrow h c) \approx 3 \times 10^{-15}$ and $\operatorname{Br}(t \rightarrow h u) \approx 2 \times 10^{-17}$ are tiny in the SM [46]. In our model the branching fraction of the top decaying to Higgs and charm is given by [47]

$$
\operatorname{Br}(t \rightarrow h c)=\frac{2\left(m_{t}^{2}-m_{h}^{2}\right)^{2} m_{W}^{2}}{g^{2}\left(m_{t}^{2}-m_{W}^{2}\right)^{2}\left(m_{t}^{2}+2 m_{W}^{2}\right)^{2}}\left(\left|g_{h c t}\right|^{2}+\left|g_{h t c}\right|^{2}+\frac{4 m_{t} m_{c}}{m_{t}^{2}-m_{h}^{2}} \operatorname{Re}\left[g_{h c t} g_{h t c}\right]\right),
$$




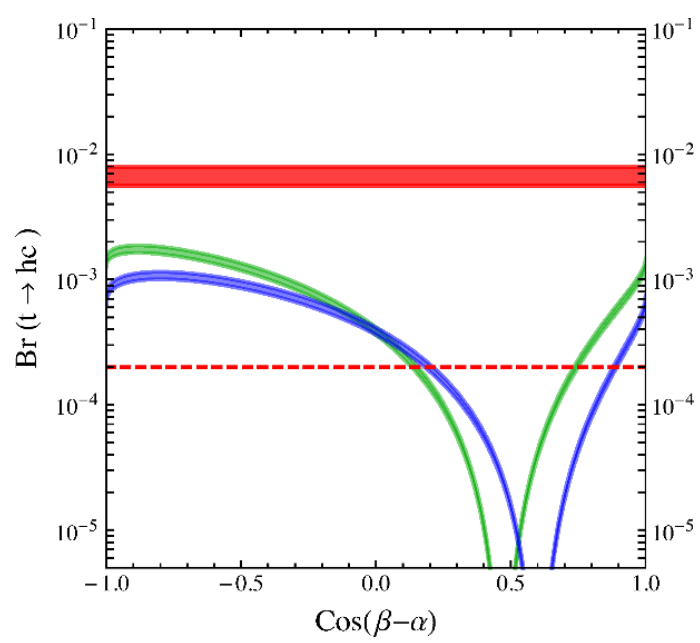

Figure 10. The plot shows $\operatorname{Br}(t \rightarrow h c)$ vs. $\cos (\beta-\alpha)$ for $\tan \beta=3(4)$ in blue (green) as well as the current exclusion limits for the $8 \mathrm{TeV}$ LHC (solid red) and projected limits at the high luminosity LHC (dashed red), respectively.

and similarly for $\operatorname{Br}(t \rightarrow h u)$ by replacing the appropriate flavor indices. Both branching ratios are parametrically of the same order, because the flavor off-diagonal couplings in equation (3.5) yield $g_{h c t} \approx g_{h u t} \propto m_{t} \varepsilon$. In figure 10 we show $\operatorname{Br}(t \rightarrow h c)$ plotted against $\cos (\beta-\alpha)$ for a range of parameter points and indicate the different predictions for $\tan \beta=$ $3(4)$ by a blue (green) band. The widths of these bands correspond to the range of values obtained by scanning over our sample set of random fundamental Yukawas. The most recent limits are $\operatorname{Br}(t \rightarrow h c)<0.56 \%$ from CMS [48] and $\operatorname{Br}(t \rightarrow h c)<0.79 \%$ from ATLAS [49] and are shown in the plot as a red band. The projected exclusion limit for $3000 \mathrm{fb}^{-1}$ at the high luminosity $\operatorname{LHC} \operatorname{Br}(t \rightarrow h c)<2 \times 10^{-4}[50]$ is indicated by a dashed red line. The plot shows that this cross section can be even above $10^{-4}$ for negative values of $\cos (\beta-\alpha)$. However, the cross section drops for the same angles for which FCNCs become small, because the same trigonometric function governs flavor off-diagonal couplings between the light Higgs to up- and down-type quarks in equation (3.5).

\section{Perturbativity, unitarity, and electroweak precision measurements}

In this section we consider perturbativity bounds, as well as constraints from the unitarity of the S matrix and electroweak precision measurements on our model. The large scalar masses implied by flavor observables and the constrained scalar potential (A.1) result in potentially large quartic couplings. Mass splittings between the different scalar mass eigenstates can in addition generate sizable contributions to the oblique parameters $S, T$ and $U$. We therefore scan over the allowed parameters, considering the various bounds described in [51]. This includes stability constraints on the Higgs potential, perturbativity bounds on the quartic scalar couplings, unitarity of the various scattering amplitudes involving scalars and the constraints from the oblique parameters. This calculation is not different from a generic two Higgs doublet model, since the oblique parameters only measure corrections to 
the gauge boson self-energies from loops of the new scalars, whose couplings are fixed by the kinetic terms $[52,53]$.

The two plots in the upper panels of figure 11 show the region in the positive $\cos (\beta-$ $\alpha)-\tan \beta$ plane in which stability and perturbativity bounds are fulfilled, and the $S$ and $T$ parameters are at most $2 \sigma$ from the best fit point, corresponding to a global $\chi^{2}$ fit obtained by the Gfitter group [54]. The upper left panel illustrates the allowed regions for degenerate scalar masses of $M \equiv M_{A}=M_{H}=M_{H^{ \pm}}=500 \mathrm{GeV}$ in light green, $M=600 \mathrm{GeV}$ in green and $M=700 \mathrm{GeV}$ in dark green. For masses $M=700 \mathrm{GeV}$ only values of $\cos (\beta-\alpha) \lesssim 0.2$ are allowed, approaching the decoupling limit. For masses $M=500 \mathrm{GeV}$ and $M=600 \mathrm{GeV}$ there is a region of parameter space in agreement with all constraints for values of $\cos (\beta-\alpha)>0.2$, that partly overlaps the region preferred by the global fit to the SM Higgs signal strengths. In the upper right panel, we show the same plot for masses $M_{H^{+}}=360-700 \mathrm{GeV}$ and $M_{A}=M_{H}=600 \mathrm{GeV}\left(M_{A}=600 \mathrm{GeV}\right.$, $M_{H}=550-650 \mathrm{GeV}$ ) in purple (dark blue). In both upper panels, we also superimpose the $2 \sigma$ contours (dashed lines) of the global Higgs fit using the ATLAS measurements of the signal strengths, that are the most stringent at present. Almost all of the right branch of the global Higgs fit can be populated for large scalar masses, while low values of $\cos (\beta-\alpha)<0.3$ are only allowed for $\tan \beta \gtrsim 4.5$.

The lower left panel shows the region allowed by all constraints discussed above for which we further demand, that the ATLAS SM Higgs signal strengths measurements are reproduced within $2 \sigma$ in the $\cos (\beta-\alpha)-M_{H^{+}}$plane for $M_{A}=M_{H}=600 \mathrm{GeV}$. The value of $\tan \beta$ is indicated by the color coding. The tiny gap at $\cos (\beta-\alpha) \approx 0.3$ is also visible in the upper left plot. For $\tan \beta \lesssim 4$ only degenerate masses $M_{A}=M_{H}=M_{H^{+}}$or a sizable mass splitting of $M_{A}-M_{H^{+}} \gtrsim 100 \mathrm{GeV}$ are allowed. We show the same plot in the lower right panel, but with a moderate mass splitting between the neutral Higgs boson masses, $M_{H}=M_{A} \pm(10-20) \mathrm{GeV}$, while keeping $M_{A}=600 \mathrm{GeV}$ fixed. ${ }^{3}$ In that case the gap around $\cos (\beta-\alpha) \approx 0.3$ becomes much more prominent.

Further, for some regions of the parameter space, one or more of the quartic couplings in the Higgs potential can become non-perturbative already at the TeV scale $\lambda_{i}(\mu=1 \mathrm{TeV}) \gtrsim$ $4 \pi$. We implement the one-loop beta functions for our model and match to the SM at an approximate average scale of the Higgs boson masses in order to estimate the scale of strong coupling. In particular for larger values $\cos (\beta-\alpha)$ and larger and degenerate masses $M_{A}=M_{H}$, the cutoff scale becomes lower. Moreover, we find that for sizable mass splittings between the charged and neutral scalars, the scale of strong coupling is in the range of $2-5 \mathrm{TeV}$. However, as mentioned in section 2 and in more detail in section 8 below, we expect the UV completion of our model to set in close to the TeV scale.

We conclude, that for fixed $M_{A}=600 \mathrm{GeV}$, two qualitatively different choices of scalar masses are compatible with electroweak precision bounds, Higgs constraints and a low $\tan \beta$ as preferred by flavor constraints. Either the scalar masses are approximately degenerate $M_{A} \approx M_{H} \approx M_{H^{+}}$or the charged scalar is considerably lighter than the neutral scalars $M_{A, H}-M_{H^{+}} \gtrsim 100 \mathrm{GeV}$. Of these possibilities, only for large mass splittings can the theory

\footnotetext{
${ }^{3}$ If the mass splittings become larger than $\left|M_{H}-M_{A}\right| \gtrsim 30 \mathrm{GeV}$, the full parameter space is excluded.
} 

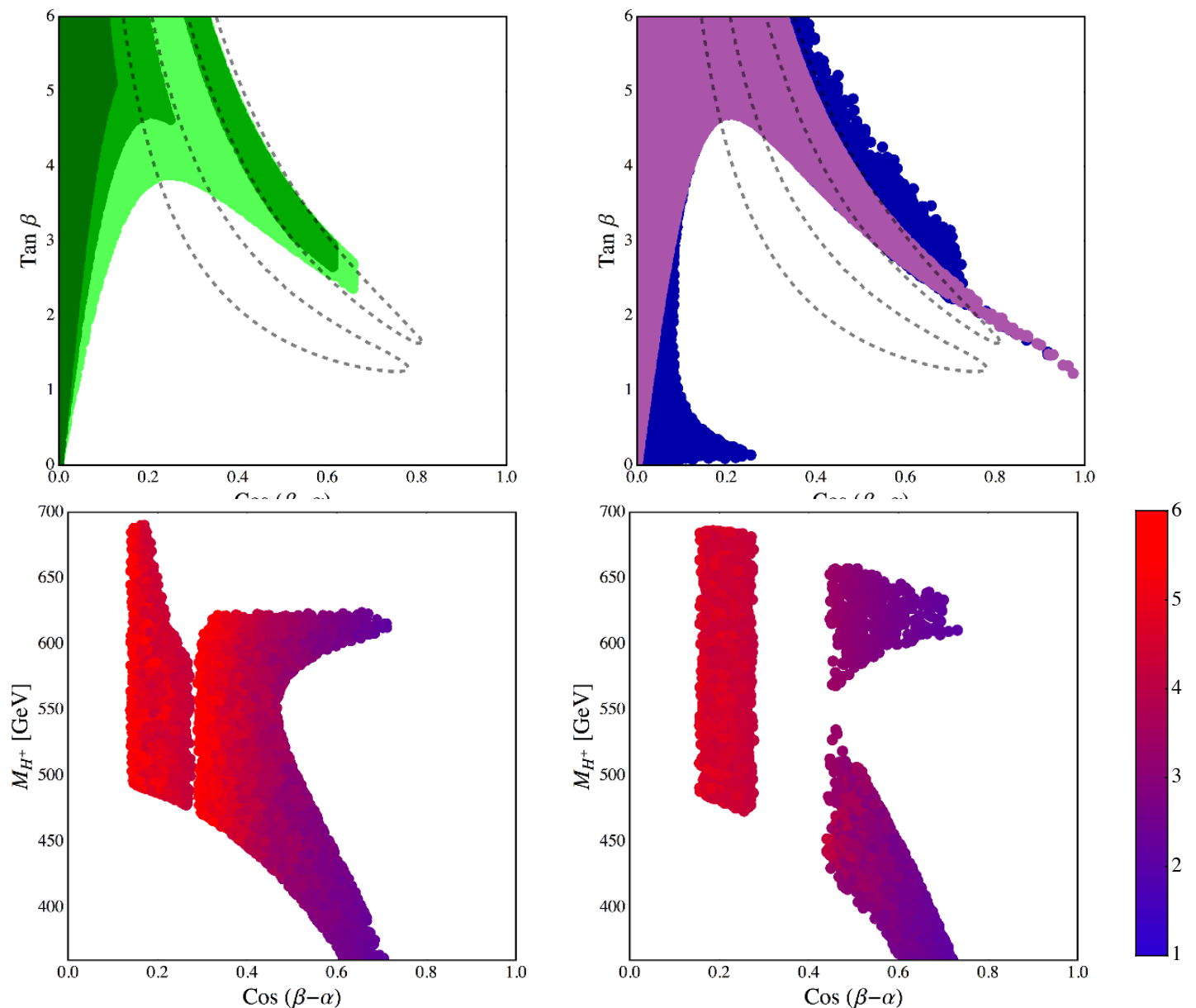

Figure 11. The upper left panel shows regions of parameter space in which the various constraints described in the text are fulfilled for scalar masses $M \equiv M_{H}=M_{A}=M_{H^{+}}=500 \mathrm{GeV}$ (light green), $M=600 \mathrm{GeV}$ (green) and $M=700 \mathrm{GeV}$ (dark green). The upper right panel shows the same plot for $M_{H^{+}}=360-700 \mathrm{GeV}$ and $M_{A}=M_{H}=600 \mathrm{GeV}\left(M_{A}=600 \mathrm{GeV}, M_{H}=550-650 \mathrm{GeV}\right)$ in purple (blue). The $2 \sigma$ contours of the ATLAS fit to Higgs measurements is shown in dashed black. The lower panels show the parameter space in the $\cos (\beta-\alpha)-M_{H^{+}}$plane in agreement with all bounds discussed in the text, including the $2 \sigma$ global fit to ATLAS data. In the lower left (right) panel we assume $M_{A}=M_{H}=600 \mathrm{GeV}\left(M_{H}=M_{A} \pm(10-20) \mathrm{GeV}\right)$, with values of $\tan \beta$ indicated by the color coding bar on the right.

be valid up to several $\mathrm{TeV}$ and in the following we will concentrate on this setup. Note, that these restrictions would be slightly relaxed if we take the fit to the CMS measurements of the Higgs signal strengths as a constraint.

Another important electroweak precision observable is the $Z b \bar{b}$ coupling. While the experimental value of the left-handed $Z b_{L} \bar{b}_{L}$ coupling is in good agreement with the SM prediction, there is a discrepancy between the measured right-handed $Z b_{R} \bar{b}_{R}$ coupling and the SM prediction, see e.g. $[54,55]$. Higher order corrections with the neutral or charged scalars in the loop can in principle affect these couplings.

The charged scalar contributions to the left-handed $Z b_{L} \bar{b}_{L}$ coupling in a two Higgs doublet model of type II can become sizable for low $\tan \beta$, inducing a bound of $t_{\beta} \gtrsim 0.5$ 
for masses of $M_{H^{ \pm}} \approx 500 \mathrm{GeV}$ [42], while corrections to the $Z b_{R} \bar{b}_{R}$ vertex are suppressed by $m_{b} / m_{t}$. In addition, the neutral scalar couplings to bottom quarks are very different from a generic two Higgs doublet model in a large range of parameter space. We define the couplings of the $Z$ boson to left-handed and right-handed bottom quarks by

$$
\mathcal{L}_{Z b b}=-\frac{e}{2 s_{W} c_{W}} Z_{\mu} \bar{b} \gamma^{\mu}\left(g^{L}\left(1-\gamma_{5}\right)+g^{R}\left(1-\gamma_{5}\right)\right) b
$$

with

$$
g^{L, R}=g_{\mathrm{SM}}^{L, R}+\delta g_{h}^{L, R}+\delta g_{A, H}^{L, R}+\delta g_{H^{ \pm}}^{L, R} .
$$

Here, $g_{\mathrm{SM}}^{L, R}$ are the SM couplings and we denote the corrections from neutral and charged Higgs exchange by $\delta g_{h}, \delta g_{A, H}$ and $\delta g_{H^{ \pm}}$, respectively. We estimate

$$
\frac{\delta g_{h}^{L}}{\delta g_{H^{ \pm}}^{L}} \propto \frac{M_{H^{ \pm}}^{2}}{m_{h}^{2}} t_{\beta}^{2} \kappa_{b}^{2} \varepsilon^{2}, \quad \frac{\delta g_{h}^{R}}{\delta g_{H^{ \pm}}^{R}} \propto \frac{M_{H^{ \pm}}^{2}}{m_{h}^{2}} \frac{\kappa_{b}^{2}}{t_{\beta}^{2}},
$$

while contributions from the heavy neutral scalars are further suppressed by $\delta g_{A, H} / \delta g_{h} \approx$ $m_{h}^{2} / M_{A, H}^{2}$ and couple with $\kappa_{b}^{A}$ and $\kappa_{b}^{H}$, as defined in the following section in equation (7.1). Neutral Higgs contributions to $g^{L}$ are therefore at least an order of magnitude smaller than the charged Higgs contributions for the region preferred by the global Higgs fit, while corrections to the right-handed coupling $g^{R}$ are at most of a similar size. We numerically estimate the light neutral Higgs contributions following [56, 57]. For $\kappa_{b}^{2}=1$, we find for the right-handed coupling $\delta g_{h}^{R} \lesssim 10^{-6} \times g_{\mathrm{SM}}^{R}$, and for the left-handed coupling $\delta g_{h}^{L} \lesssim 10^{-6} \times g_{\mathrm{SM}}^{L}$, which is many orders of magnitude too small in order to explain the anomalous $Z b_{R} b_{R}$ coupling. In order to improve the fit with respect to the SM, contributions of the order of $0.2 \%$ to $g_{\mathrm{SM}}^{L}$ and $2 \%-20 \%$ to $g_{\mathrm{SM}}^{R}$ (depending on the sign) are necessary [58]. The neutral Higgs contributions to the $Z b \bar{b}$ vertex can therefore be safely neglected. It should be noted, that fermionic mixing effects in the UV completion of this model can affect both the oblique parameters and the $Z b \bar{b}$ vertex. These however depend sensitively on the exact realization of the UV completion, which is beyond the scope of this paper.

\section{Collider searches for heavy extra scalars}

Our model features heavy new scalars beyond the SM, namely the neutral scalar Higgs $H$, the pseudoscalar $A$ and the charged Higgs $H^{ \pm}$. Their masses are bound to be less than $700 \mathrm{GeV}$ by perturbativity, and various flavor constraints set lower bounds on their masses as discussed in section 5. In this section we consider the latest ATLAS and CMS bounds on new neutral and charged Higgs bosons.

\subsection{Couplings and total width of heavy scalars}

Similar to the case of the light scalar, the couplings of the heavy scalar $H$ and pseudoscalar $A$ to quarks - with the exception of the top quark - differ from the couplings in a two Higgs doublet model. The couplings to gauge bosons are instead the same as in a two 


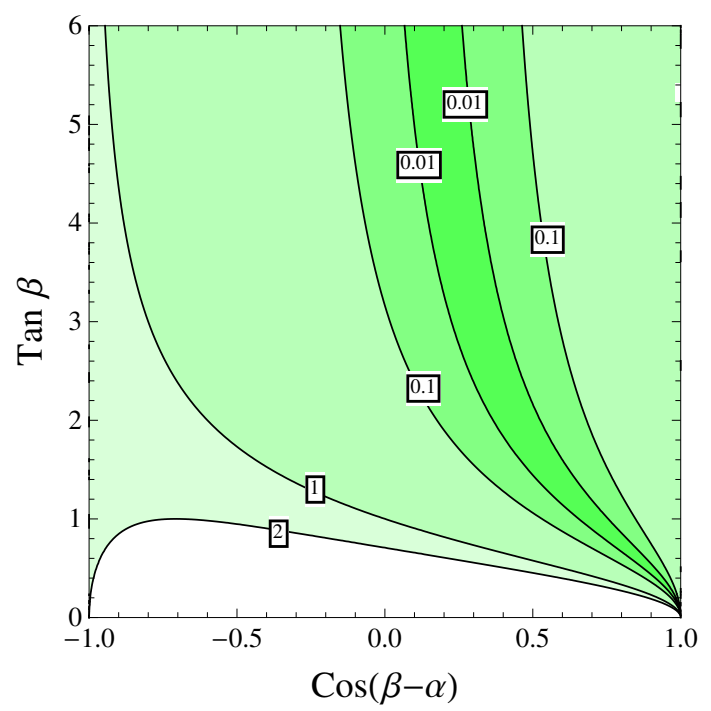

Figure 12. Contours of $\left(\kappa_{t}^{H}\right)^{2}$ in the $\cos (\beta-\alpha)-\tan \beta$ plane. A suppression of the coupling with respect to the $\mathrm{SM}$ is achieved in the darker shaded area.

Higgs doublet model. Specifically, the couplings of $H$ and $A$ to gauge bosons and third generation quarks normalized to the SM as in (4.2), read

$$
\begin{array}{rlrl}
\kappa_{t}^{H} & =c_{\beta-\alpha}-\frac{s_{\beta-\alpha}}{t_{\beta}}, & \kappa_{b}^{H} & =3 c_{\beta-\alpha}+s_{\beta-\alpha}\left(2 t_{\beta}-\frac{1}{t_{\beta}}\right), \\
\kappa_{t}^{A}=\frac{1}{t_{\beta}}, & \kappa_{b}^{A}=2 t_{\beta}+\frac{1}{t_{\beta}},
\end{array}
$$

where $t, b$ and $V$ denote the rescaling factor for top, bottom and vector boson couplings, respectively. Since $\left(\kappa_{t}^{H}\right)^{2}$ is relevant for the gluon fusion production of the heavy Higgs boson $H$, its parametric dependence is essential and we illustrate it in figure 12. Both flavor diagonal and flavor changing couplings of $H$ and $A$ involving the charm quark, are given by

$$
\begin{array}{lll}
\kappa_{c}^{H}=3 c_{\beta-\alpha}+s_{\beta-\alpha}\left(t_{\beta}-\frac{2}{t_{\beta}}\right), & \kappa_{t c}^{H}=\left(2 c_{\beta-\alpha}+s_{\beta-\alpha}\left(t_{\beta}-\frac{1}{t_{\beta}}\right)\right) \cdot \varepsilon, \\
\kappa_{c}^{A}=\frac{2}{t_{\beta}}+t_{\beta}, & \kappa_{t c}^{A}=\left(t_{\beta}+\frac{1}{t_{\beta}}\right) \cdot \varepsilon,
\end{array}
$$

where $\kappa_{t c}^{A}$ and $\kappa_{t c}^{H}$ are defined according to equation (7.7) below. As discussed at the end of section 2 , we define the couplings to taus as

$$
\kappa_{\tau}^{H}=\kappa_{b}^{H}, \quad \kappa_{\tau}^{A}=\kappa_{b}^{A} .
$$

The couplings of the charged Higgs $H^{+}$to fermions are the same as in a two Higgs doublet model of type II. Similarly, all self-couplings between the scalars are the same as in a generic two Higgs doublet model. The coupling between the heavy scalar $H$ and the light 

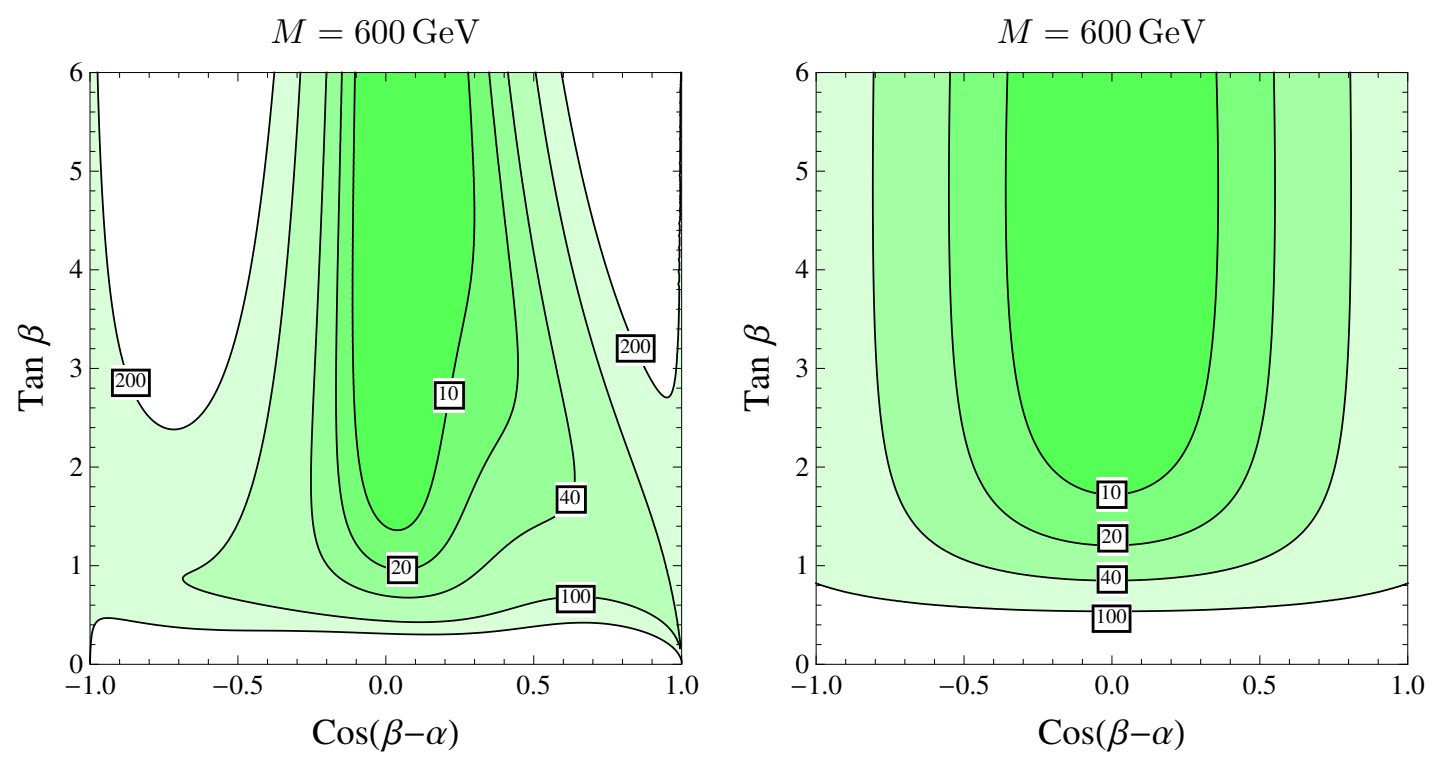

Figure 13. The plot shows the parametric dependence of the total width for the heavy Higgs $H$ (left panel) and total width for the pseudoscalar $A$ (right panel) for $M=600 \mathrm{GeV}$. The contours, labeled in $\mathrm{GeV}$, show lines of constant width.

Higgs $h$ is of particular interest for the following analysis and reads [5, 9, 12]

$$
g_{H h h}=\frac{c_{\beta-\alpha}}{v}\left[\left(3 M_{A}^{2}-2 m_{h}^{2}-M_{H}^{2}\right)\left(c_{2(\beta-\alpha)}-\frac{s_{2(\beta-\alpha)}}{t_{2 \beta}}\right)-M_{A}^{2}\right] .
$$

Finally, the couplings between two Higgs bosons and one gauge boson read [4]

$$
\begin{aligned}
g_{A h Z} & =\frac{g}{2 \cos \theta_{W}} c_{\beta-\alpha}, & g_{A H Z} & =\frac{g}{2 \cos \theta_{W}} s_{\beta-\alpha}, \\
g_{h H^{+} W^{-}} & =\frac{g}{2} c_{\beta-\alpha}, & g_{H H^{+} W^{-}} & =\frac{g}{2} s_{\beta-\alpha} .
\end{aligned}
$$

Further, we define the total widths for $H, A$, and $H^{+}$, including all relevant and kinematically accessible decay channels (no off-shell decays are relevant in the regions we will consider)

$$
\begin{aligned}
\Gamma_{H}= & \Gamma(H \rightarrow W W)+\Gamma(H \rightarrow Z Z)+\Gamma(H \rightarrow h h)+\Gamma(H \rightarrow A Z)+\Gamma\left(H \rightarrow H^{+} W^{-}\right) \\
& +\Gamma(H \rightarrow t \bar{t})+\Gamma(H \rightarrow b \bar{b})+\Gamma(H \rightarrow c \bar{c})+\Gamma(H \rightarrow t \bar{c})+\Gamma(H \rightarrow g \bar{g}) \\
& +\Gamma\left(H \rightarrow \tau^{+} \tau^{-}\right) \\
\Gamma_{A}= & \Gamma(A \rightarrow h Z)+\Gamma(A \rightarrow H Z)+\Gamma\left(A \rightarrow H^{+} W^{-}\right)+\Gamma(A \rightarrow t \bar{t})+\Gamma(A \rightarrow b \bar{b}) \\
& +\Gamma(A \rightarrow c \bar{c})+\Gamma(A \rightarrow t \bar{c})+\Gamma(A \rightarrow g \bar{g})+\Gamma\left(A \rightarrow \tau^{+} \tau^{-}\right), \\
\Gamma_{H^{+}}= & \Gamma\left(H^{+} \rightarrow h W^{+}\right)+\Gamma\left(H^{+} \rightarrow H W^{+}\right)+\Gamma\left(H^{+} \rightarrow A W^{+}\right)+\Gamma\left(H^{+} \rightarrow t \bar{b}\right) \\
& +\Gamma\left(H^{+} \rightarrow \tau \bar{\nu}\right) .
\end{aligned}
$$

Note that, besides the usual decay channels the flavor violating channel $\Gamma(\Phi \rightarrow c \bar{t})$ with 
$\Phi=H, A$ appears in (7.6). This channel is characteristic for our model and we therefore give the partial width explicitely

$$
\Gamma(\Phi \rightarrow c \bar{t})=\frac{3}{8 \pi}\left(\kappa_{t c}^{\Phi}\right)^{2} \frac{m_{t}^{2}}{v^{2}} M_{\Phi} \sqrt{\lambda\left(1, \frac{m_{t}^{2}}{M_{\Phi}^{2}}, \frac{m_{c}^{2}}{M_{\Phi}^{2}}\right)}\left\{\begin{array}{lll}
\left(\frac{\left(m_{t}-m c\right)^{2}}{M_{A}^{2}}-1\right) & \text { for } & M_{\Phi}=M_{A}, \\
\left(1-\frac{\left(m_{t}+m c\right)^{2}}{M_{H}^{2}}\right) & \text { for } & M_{\Phi}=M_{H},
\end{array}\right.
$$

with

$$
\lambda(x, y, z)=x^{2}+y^{2}+z^{2}-2 x y-2 x z-2 y z .
$$

The parametric dependence of the total width for the scalar (pseudoscalar) Higgs boson is illustrated in the left (right) panel of figure 13 for $M=M_{A}=M_{H}=M_{H^{+}}=600 \mathrm{GeV}$. For large regions of parameter space the total width becomes large. In particular, for $\tan \beta>1$ and $|\cos (\beta-\alpha)|>\mathcal{O}(0.5)$ values of $\mathcal{O}(100) \mathrm{GeV}$ can be obtained, such that finite width effects need to be taken into account. The charged Higgs can also have a sizable branching ratio $\operatorname{Br}\left(H^{+} \rightarrow h W^{+}\right)$, which can become the dominant decay channel for sufficiently large $\cos (\beta-\alpha)$. In appendix $\mathrm{E}$, in figures $23-25$ we show the branching ratios for all Higgs bosons for specific benchmark scenarios to be discussed later.

\subsection{Analysis of production and decay channels}

In the following we study the impact of searches for heavy Higgs bosons at ATLAS and CMS. To this end, we compute the production cross section and various decay rates for the heavy Higgs bosons. We generate the gluon-fusion production cross section at nextto-leading order (NLO) using HIGLU [59], taking into account the contributions of the bottom quark loop and use the leading order expressions for the partial decay width with the appropriate couplings of our model [60,61]. When relevant, we also consider the vector-boson fusion production cross section, using the values quoted in [62, 63]. For charged Higgs production we use the NLO results in [64]. In the following we will assume $M=M_{A}=M_{H}=M_{H^{+}}$, if not specified otherwise, and we discuss in detail the effects of a splitting between the neutral and charged Higgs boson masses.

One of the most interesting channels for the discovery of the pseudoscalar Higgs boson, involves the $A \rightarrow h Z$ decay, because the corresponding branching ratio becomes dominant for sizable values of $\cos (\beta-\alpha)$. There are several experimental studies constraining $\sigma(g g \rightarrow$ $A) \times \operatorname{Br}(A \rightarrow h Z)$, with the light Higgs further decaying into bottom quarks [65, 66], tau leptons [65], as well as multi-leptons [49].

The predictions of our model for both $\sigma(g g \rightarrow A) \times \operatorname{Br}(A \rightarrow h Z)$ and $\sigma(g g \rightarrow A) \times$ $\operatorname{Br}\left(A \rightarrow h Z \rightarrow \ell^{+} \ell^{-} b \bar{b}\right)$ are presented in figure 14 in the left and right panel, respectively. For the decay rate $\Gamma(h \rightarrow b \bar{b})$, NLO corrections are sizable and therefore we include them in our analysis by setting

$$
\Gamma(h \rightarrow b \bar{b})=0.57 \kappa_{b}^{2} \Gamma_{h}^{\mathrm{SM}},
$$

where we use $\Gamma_{h}^{\mathrm{SM}}=4.07 \mathrm{MeV}[32]$ and $\operatorname{Br}(Z \rightarrow \ell \ell)=6.729 \%$ for $\ell^{-}=e^{-}, \mu^{-}$[32]. In the left panel of figure 14 we show the contours of $\sigma(g g \rightarrow A) \times \operatorname{Br}(A \rightarrow h Z)$ in picobarn for $8 \mathrm{TeV}$ proton-proton $(p p)$ collisions in the $\cos (\beta-\alpha)-\tan \beta$ plane for $M=600 \mathrm{GeV}$. 

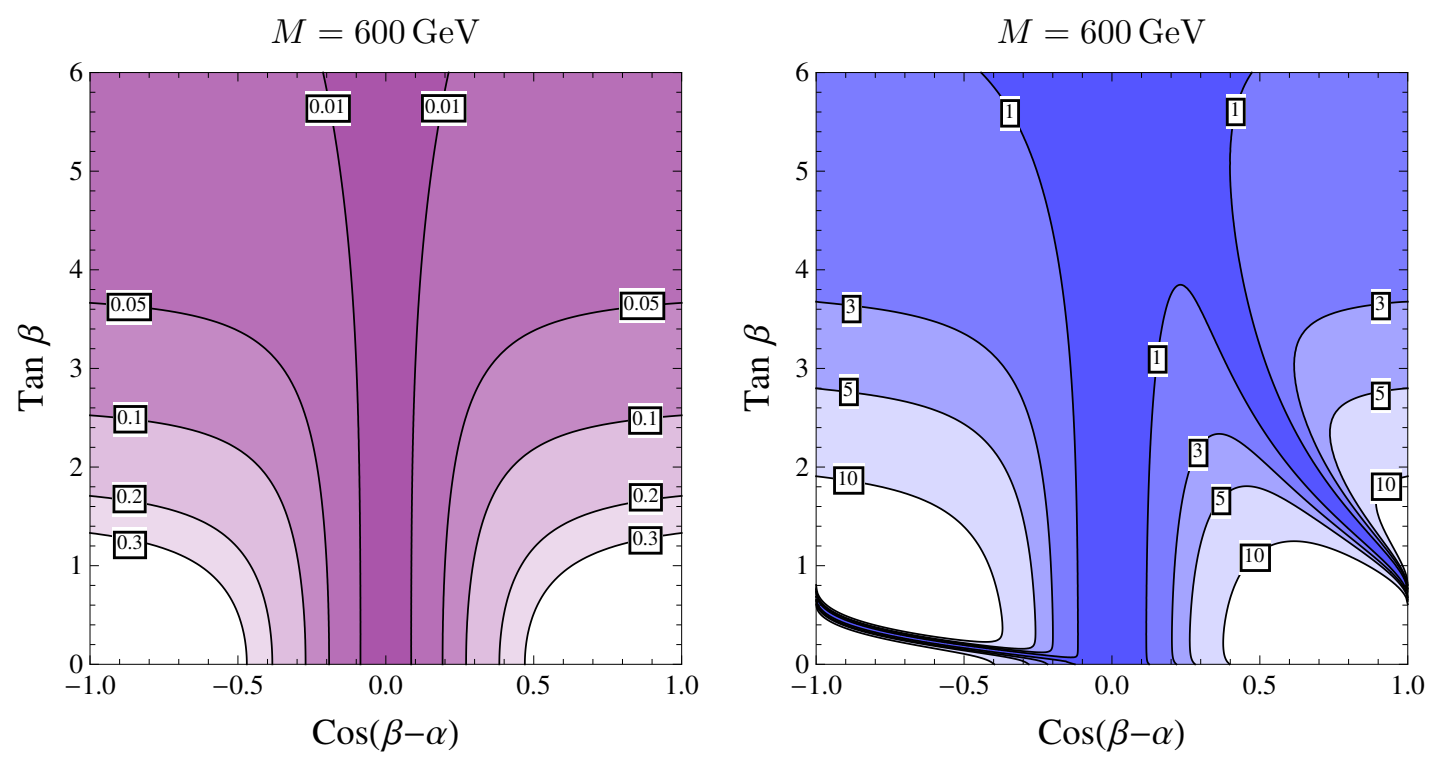

Figure 14. We show contours of constant $\sigma(g g \rightarrow A) \times \operatorname{Br}(A \rightarrow h Z)$ in picobarn (left panel) and $\sigma(g g \rightarrow A) \times \operatorname{Br}(A \rightarrow h Z \rightarrow \ell \ell b \bar{b})$ in femtobarn (right panel) for $8 \mathrm{TeV} p p$ collisions and $M=600 \mathrm{GeV}$.

The shape of the contours follows naturally from the fact that the branching ratio scales as $\cos (\beta-\alpha)^{2}$, while the production cross section depends only on $\tan \beta$. This is no different than in a generic two Higgs doublet model [5, 49], but it is particularly relevant in our model, since it cannot live close to the decoupling limit, as discussed in section 4 . The experimental exclusion bounds from [49] constrain $\sigma(g g \rightarrow A) \times \operatorname{Br}(A \rightarrow h Z)$ considering a multi-lepton final state, but the study is only performed for pseudoscalars with masses up to $M_{A}<360 \mathrm{GeV}$.

In the right panel of figure 14 we show the contours of $\sigma(g g \rightarrow A) \times \operatorname{Br}(A \rightarrow h Z \rightarrow$ $\left.\ell^{+} \ell^{-} b \bar{b}\right)$ in femtobarn for $8 \mathrm{TeV} p p$ collisions in the $\cos (\beta-\alpha)-\tan \beta$ plane and $M=$ $600 \mathrm{GeV}$. Two branches with suppressed values for $\sigma(g g \rightarrow A) \times \operatorname{Br}(A \rightarrow h Z \rightarrow \ell \ell b \bar{b})$ appear. The first branch is the decoupling or alignment limit, where $g_{A h Z}$ vanishes. The second branch is given by the region for which the coupling of the light Higgs $h$ to bottom quarks becomes small.

We consider the measurement of $\sigma(g g \rightarrow A) \times \operatorname{Br}(A \rightarrow h Z) \times \operatorname{Br}(h \rightarrow b \bar{b})$ by ATLAS [65] and the measurement of $\sigma(g g \rightarrow A) \times \operatorname{Br}\left(A \rightarrow h Z \rightarrow \ell^{+} \ell^{-} b \bar{b}\right)$ by CMS [66] with $\ell^{-}=$ $e^{-}, \mu^{-}$. Both experiments give their bounds assuming narrow width approximation for the heavy scalar. In figure 15 we compare these bounds (blue curves) from both experiments for equal masses of the heavy scalars with $M=500 \mathrm{GeV}$ (dotted) and $M=600 \mathrm{GeV}$ (dashed). For both mass choices the ATLAS measurement gives a stronger bound. For $M=500 \mathrm{GeV}$, substantial regions of the model parameter space are ruled out, however for $M=600 \mathrm{GeV}$ the model is considerably less constrained. The right panel of figure 15 also shows the ATLAS bounds [65] of $\sigma(g g \rightarrow A) \times \operatorname{Br}(A \rightarrow h Z)$ with the light Higgs $h$ decaying further to tau leptons. The corresponding bounds are shown as green contours for 

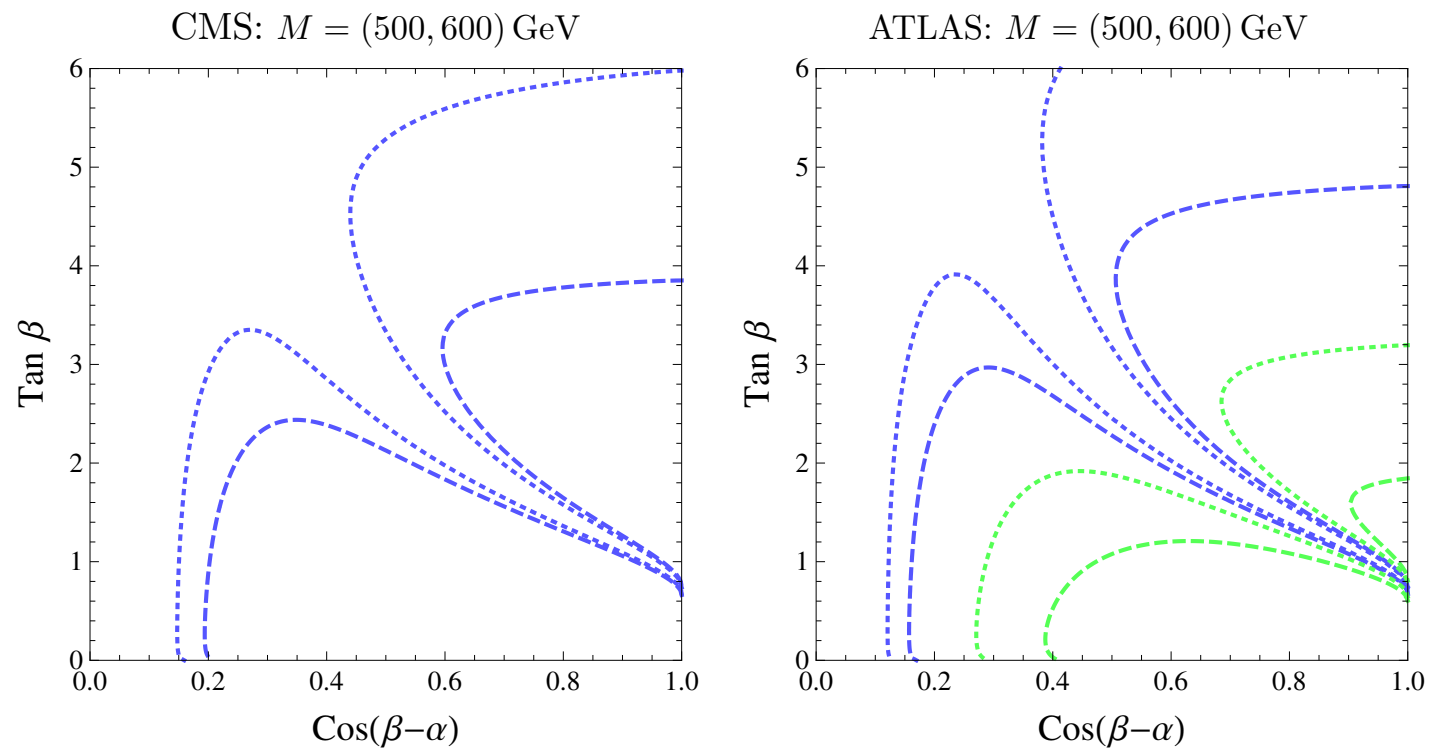

Figure 15. In the left panel we show current exclusion bounds for $\sigma(g g \rightarrow A) \times \operatorname{Br}(A \rightarrow h Z \rightarrow \ell \ell b \bar{b})$ based on the CMS data [66]. In the right panel we show exclusion bounds for $\sigma(g g \rightarrow A) \times \operatorname{Br}(A \rightarrow$ $h Z) \times \operatorname{Br}(h \rightarrow b \bar{b})$ (blue) and $\sigma(g g \rightarrow A) \times \operatorname{Br}(A \rightarrow h Z) \times \operatorname{Br}\left(h \rightarrow \tau^{+} \tau^{-}\right)$(green) based on ATLAS data [65]. In both plots we assume equal scalar masses, $M=500 \mathrm{GeV}$ (dotted) and $M=600 \mathrm{GeV}$ (dashed), and narrow-width approximation. The region below and to the right of the curves is excluded.

$M=500 \mathrm{GeV}$ (dotted) and $M=600 \mathrm{GeV}$ (dashed). These constraints are substantially weaker than the corresponding bounds for the $h \rightarrow b \bar{b}$ decay.

In the following we consider the impact of finite width effects on the previous bounds. In the right panel of figure 16, we show the rescaling factor for the cross section times branching ratio due to finite width effects, extrapolated from the CMS analysis [66], for $M_{A}=500(600) \mathrm{GeV}$ in pink (green). In the left panel of figure 16 we first show for comparison the exclusion bound from ATLAS data for $M=600 \mathrm{GeV}$ in the narrow width approximation. Under the assumption that the scaling effects for ATLAS and CMS are similar and assuming sensitivity up to a total width of $\Gamma_{A} \simeq 100 \mathrm{GeV}$, we consider finite width effects for each point in the $\cos (\beta-\alpha)-\tan \beta$ plane and reinterpret the ATLAS results (solid, blue line in the left panel of figure 16). Although finite width effects significantly weaken the exclusion bound, this channel remains the most promising discovery channel at the LHC run II. The bound is further relaxed in the case of a mass splitting, $M_{A} \gg$ $M_{H^{+}}\left(M_{H}\right)$, such that the decay channels $A \rightarrow H^{+} W^{-}(H Z)$ open up. Our discussion in section 6 showed that such a mass splitting is only allowed between the pseudoscalar and the charged Higgs boson. We present the bound for $\sigma(g g \rightarrow A) \times \operatorname{Br}(A \rightarrow h Z) \times \operatorname{Br}(h \rightarrow b \bar{b})$ including finite width effects for $M_{A}=M_{H}=600 \mathrm{GeV}$ and $M_{H^{+}}=400 \mathrm{GeV}$ in the left panel of figure 16 (black).

In the following we will consider the experimental bounds from searches for the neutral CP-even Higgs boson $H$. There are two channels of particular interest, the CP even scalar decaying into light Higgs bosons $H \rightarrow h h$ and the CP even scalar decaying to vector bosons $H \rightarrow V V$ with $V=W, Z$. 

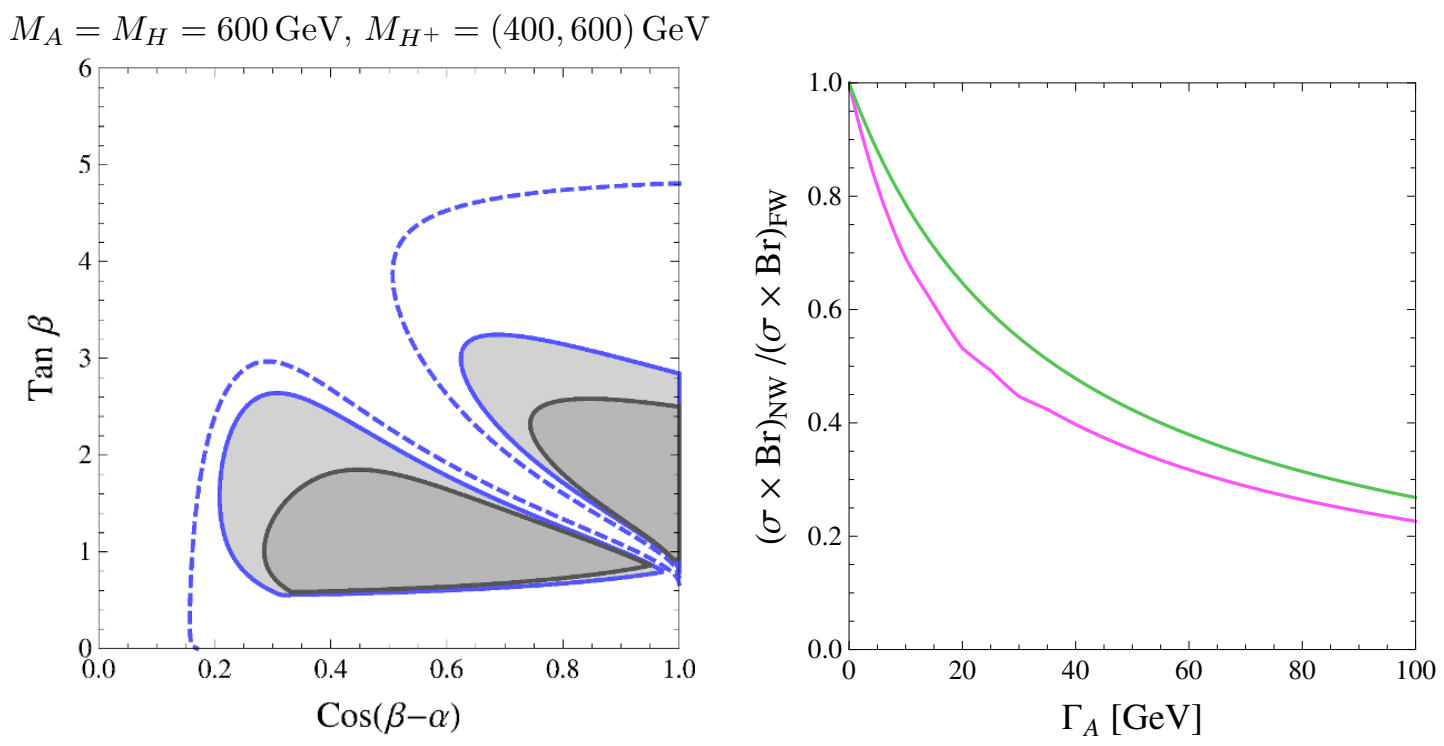

Figure 16. In the left panel, we show the exclusion contour for $\sigma(g g \rightarrow A) \times \operatorname{Br}(A \rightarrow h Z) \times \operatorname{Br}(h \rightarrow$ $b \bar{b})$ for $M=600 \mathrm{GeV}$ in the narrow width (NW) approximation (dashed blue) and taking into account finite width (FW) effects (solid blue). The black contour additionally shows mass splitting effects, assuming $M_{A}=M_{H}=600 \mathrm{GeV}$ and $M_{H^{+}}=400 \mathrm{GeV}$. The shaded region inside each contour depicts the excluded area. The right panel shows the rescaling factor due to $\mathrm{FW}$ effects with respect to the NW approximation extrapolated from the CMS analysis [66], for $M_{A}=500(600) \mathrm{GeV}$ in pink (green).

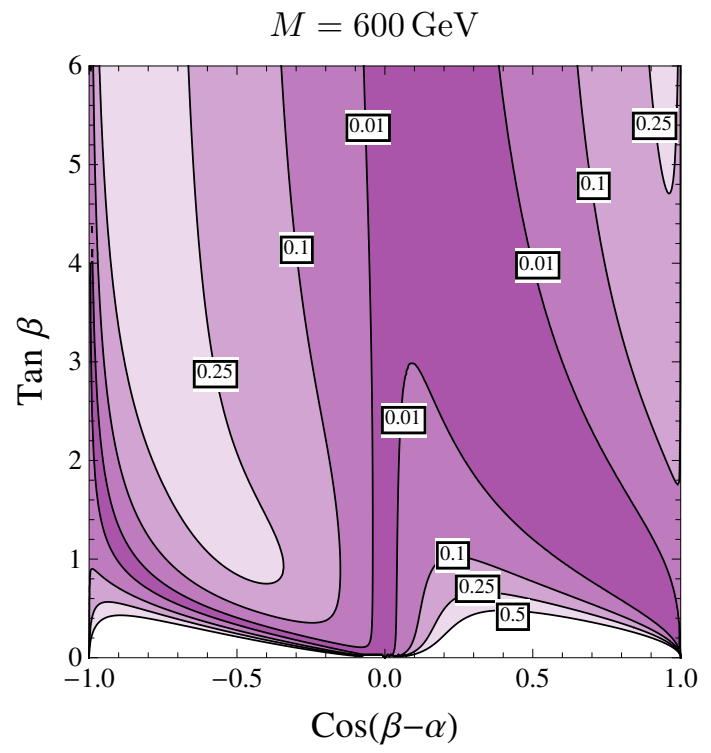

Figure 17. Model predictions for the contours of $\sigma(g g \rightarrow H) \times \operatorname{Br}(H \rightarrow h h)$ in picobarn for $8 \mathrm{TeV}$ $p p$ collisions and heavy scalar masses $M=600 \mathrm{GeV}$. 
In figure 17 we present predictions for $\sigma(g g \rightarrow H) \times \operatorname{Br}(H \rightarrow h h)$ in picobarn for $8 \mathrm{TeV} p p$ collisions in the $\cos (\beta-\alpha)-\tan \beta$ plane for $M=600 \mathrm{GeV}$. From (7.4) we observe that the self coupling $g_{H h h}$ is proportional to $\cos (\beta-\alpha)$ and has an explicit $M_{A}$ dependence. For $\cos (\beta-\alpha) \geq 0$ we observe two branches of contours with suppressed $\sigma \times \mathrm{Br}$. The first branch approaches zero at $\cos (\beta-\alpha)=0$, and for the second branch both the coupling $g_{H h h}$ and the production cross section become small. Predictions for $\sigma(g g \rightarrow H) \times \operatorname{Br}(H \rightarrow h h)$ are comparable to the ones in a generic two Higgs doublet model of type II [5]. Similar to the pseudoscalar case, the experimental exclusion bounds for $\sigma(g g \rightarrow H) \times \operatorname{Br}(H \rightarrow h h)$ [49] are only available up to $M_{H}<360 \mathrm{GeV}$. However for the $\mathrm{CP}$ even Higgs, the model predictions seem to be much below the present experimental sensitivity.

The most important search channel for the heavy $\mathrm{CP}$ even neutral Higgs boson $H$ is the inclusive production with subsequent decay of $H \rightarrow V V$ with $V=W, Z$. In our specific model there is an interesting region of parameter space in which the vector boson fusion production is competitive with the gluon fusion production due to the behavior of $\kappa_{t}^{H}$. Normalized to the corresponding SM Higgs production and decay processes for a SM Higgs of mass $M_{H}$, we have for gluon fusion and vector boson fusion production processes, respectively,

$$
\begin{aligned}
\frac{\sigma(g g \rightarrow H) \times \operatorname{Br}(H \rightarrow V V)}{(\sigma(g g \rightarrow H) \times \operatorname{Br}(H \rightarrow V V))_{\mathrm{SM}}} & =\left(\kappa_{t}^{H}\right)^{2}\left(1+\xi_{b}^{H} \frac{\kappa_{b}^{H}}{\kappa_{t}^{H}}\right)^{2}\left(\kappa_{V}^{H}\right)^{2} \frac{\Gamma_{H}^{\mathrm{SM}}}{\Gamma_{H}}, \\
\frac{\sigma(p p \rightarrow q q H) \times \operatorname{Br}(H \rightarrow V V)}{(\sigma(p p \rightarrow q q H) \times \operatorname{Br}(H \rightarrow V V))_{\mathrm{SM}}} & =\left(\kappa_{V}^{H}\right)^{4} \frac{\Gamma_{H}^{\mathrm{SM}}}{\Gamma_{H}},
\end{aligned}
$$

where $\xi_{b}^{H}$ denotes the correction from a bottom quark in gluon fusion with respect to the leading top contribution. We take the SM total width $\Gamma_{H}^{S M}$ for a heavy Higgs of mass $M_{H}$ from the LHC Higgs Cross section Working Group [62, 63, 67].

In figure 18 we present theoretical predictions for contours of inclusive heavy neutral CP even Higgs production (left panel) and vector boson fusion production (right panel) with subsequent decay into $H \rightarrow V V$, using (7.10) and (7.11), for $M=M_{A}=M_{H}=M_{H^{+}}=$ $600 \mathrm{GeV}$. The vector boson fusion is governed by $\kappa_{V}^{H}$ and becomes strongly suppressed for small $\cos (\beta-\alpha)$. The gluon fusion production mode in (7.10) is suppressed for small values of $\kappa_{t}^{H}$ or for small $\kappa_{V}^{H}$ and this effect shows in the inclusive production mode above. We observe that for small $\kappa_{t}^{H}$, both production cross sections become competitive. The theory prediction for these two observables differs from a two Higgs doublet model of type II only by the different scaling of the width $\Gamma_{H}$ and the contribution of the bottom quark to gluon fusion, which is small for $\tan \beta \sim \mathcal{O}(1)$.

The CMS collaboration has reported updated results from an inclusive search for a heavy Higgs decaying into $W^{+} W^{-}$and $Z Z$ in the range of $M_{H}=145-1000 \mathrm{GeV}$ [68]. They consider both fully leptonic and semileptonic final states. In figure 19 we illustrate those bounds for $M=M_{A}=M_{H}=M_{H^{+}}$with $M=500 \mathrm{GeV}$ (dotted) and $M=600 \mathrm{GeV}$ (solid). We observe that this search mode is competitive with the bounds obtained from the $A \rightarrow h Z$ channel. We note that for the neutral CP even Higgs analysis no finite width 

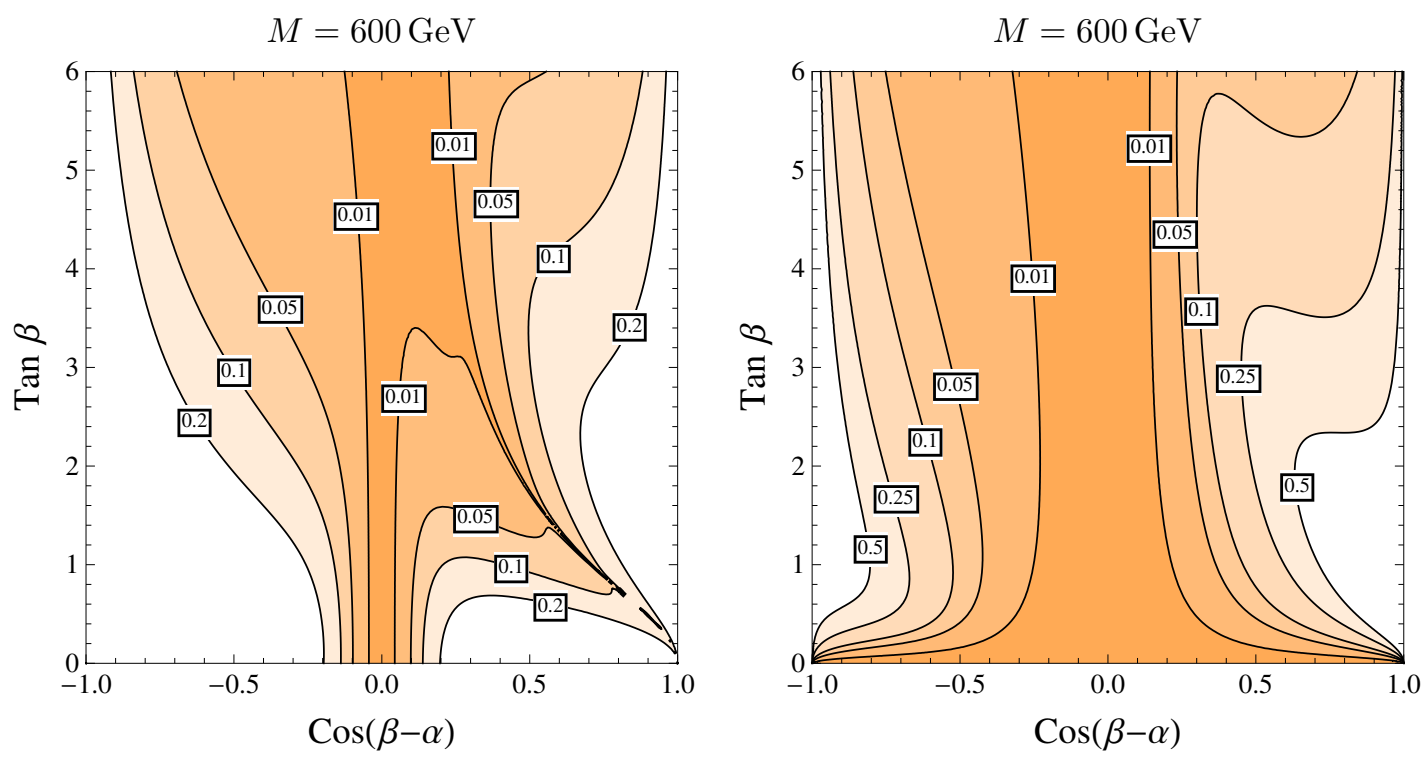

Figure 18. Contours of $\sigma(p p \rightarrow H+X) \times \operatorname{Br}(H \rightarrow V V) /(\sigma(p p \rightarrow H+X) \times \operatorname{Br}(H \rightarrow V V))_{\mathrm{SM}}$ (left panel) and $\sigma(p p \rightarrow q q H) \times \operatorname{Br}(H \rightarrow V V) /(\sigma(p p \rightarrow q q H) \times \operatorname{Br}(H \rightarrow V V))_{\mathrm{SM}}$ (right panel). The heavy scalar masses are set to $M=600 \mathrm{GeV}$.

effects have been taken into account, although we expect sizable finite width effects in a large region of parameter space, compare the left panel of figure 13 .

The CMS collaboration also performed an analysis for a heavy neutral Higgs boson decaying into $W^{+} W^{-}$in vector boson fusion production channel in the mass range $M_{H}=$ $110-600 \mathrm{GeV}$ [69]. The observed signal significance is close to the SM prediction for a Higgs of $M_{H}=300-600 \mathrm{GeV}$, and hence from the right panel of figure 18 it follows that there is no sensitivity to the preferred parameter region from this search.

Searches for heavy charged Higgs bosons have been performed by both ATLAS and CMS collaborations. In particular, they searched for production modes in association with a single top, $\sigma\left(b g \rightarrow H^{-} t\right)$, or top and bottom quarks, $\sigma\left(g g \rightarrow H^{-} t \bar{b}\right)$, with subsequent decays into third generation fermions: $H^{-} \rightarrow \bar{t} b$ and $H^{-} \rightarrow \tau \nu_{\tau}$ [70-72]. The most recent limits are

$$
\begin{aligned}
& \operatorname{Br}\left(H^{-} \rightarrow \tau \nu\right)<0.153 \mathrm{pb}-0.026 \mathrm{pb} \quad \text { for } \quad M_{H^{+}}=300-600 \mathrm{GeV} \text {, } \\
& \operatorname{Br}\left(H^{-} \rightarrow \bar{t} b\right)<6 \mathrm{pb}-4 \mathrm{pb} \quad \text { for } \quad M_{H^{+}}=300-600 \mathrm{GeV} \text {, }
\end{aligned}
$$

assuming $\operatorname{Br}\left(H^{-} \rightarrow \tau \nu\right)=100 \%$ and $\operatorname{Br}\left(H^{-} \rightarrow \bar{t} b\right)=100 \%$, respectively. These values are below the expected production cross section, $\sigma\left(p p \rightarrow H^{-} t(b)\right)=70 \mathrm{fb}-6 \mathrm{fb}$ for $M_{H^{+}}=$ $300 \mathrm{GeV}-600 \mathrm{GeV}$ and $\tan \beta \approx 2$ (lower values of the production cross section occur for $2<\tan \beta<6$ ) [64]. A heavy charged Higgs boson is therefore not constrained for the parameter region of interest, through current direct search limits.

For a heavy charged Higgs $M_{H^{+}} \approx 360-400 \mathrm{GeV}, \cos (\beta-\alpha) \gtrsim 0.3(0.2)$ and $\tan \beta=$ $2(4)$, the decay channel $H^{+} \rightarrow h W^{+}$dominates over $H^{+} \rightarrow t \bar{b}$. The branching ratio can become as large as $\operatorname{Br}\left(H^{+} \rightarrow h W^{+}\right) \approx 85 \%$ for $\tan \beta=2.5, \cos (\beta-\alpha)=0.6$. For a lighter 


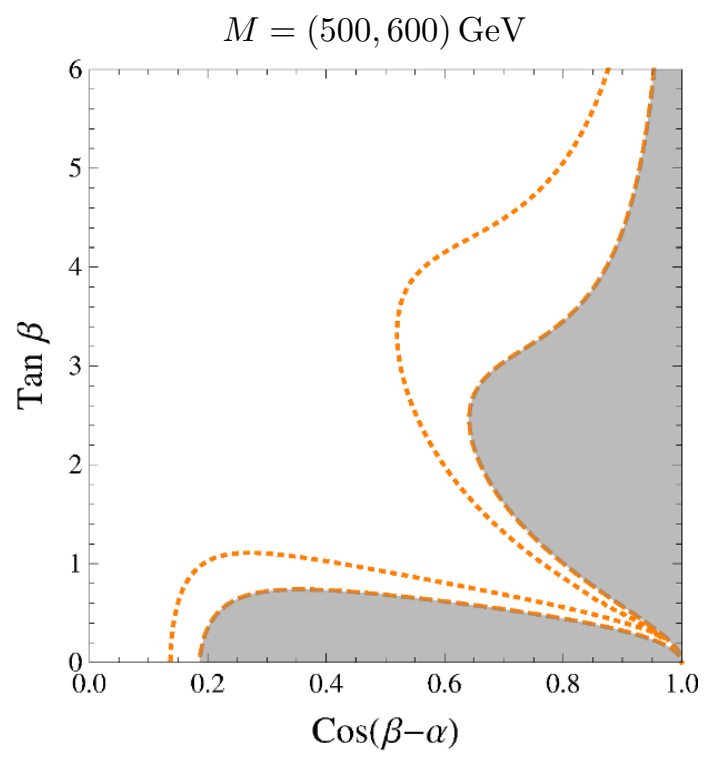

Figure 19. Exclusion bounds for $\sigma(p p \rightarrow H+X) \times \operatorname{Br}(H \rightarrow V V) /(\sigma(p p \rightarrow H+X) \times \operatorname{Br}(H \rightarrow$ $V V))_{\mathrm{SM}}$ of CMS [68] for $M=500 \mathrm{GeV}$ (dotted) and $M=600 \mathrm{GeV}$ (dashed).

charged Higgs, this is slightly less pronounced and we find $\operatorname{Br}\left(H^{+} \rightarrow h W^{+}\right) \approx 70 \%$ for $\tan \beta=2.5, \cos (\beta-\alpha)=0.6$ and $M_{H^{+}}=400 \mathrm{GeV}$.

\section{Origin of the effective Yukawa couplings}

In this section we present an example of the origin of the effective Yukawa couplings at the $\mathrm{TeV}$ scale for the bottom quark sector. Similar considerations can explain the generation of the other effective light quark Yukawa couplings in our model. A complete description of the UV completion is beyond the scope of this paper.

A possible completion of the Froggatt Nielsen model may introduce new colored vectorlike fermions or additional scalar doublets [73], whose masses determine the suppression scale $\Lambda$ in the expansion parameter (2.5). Since in our model the flavor breaking scale is identified with the electroweak scale and the expansion parameter is fixed by the ratio of bottom and top quark masses $\varepsilon=m_{b} / m_{t}$, the UV scale is constrained to be of the order of $\Lambda \sim 1 \mathrm{TeV}$.

The relevant operators that would provide a UV completion for the bottom Yukawa interactions are

$$
\mathcal{L}_{\mathrm{UV}}=y_{1} \bar{b}_{L} H_{d} \eta_{R}+y_{2} \bar{\eta}_{R} H_{u} \psi_{L}+y_{3} \bar{\psi}_{L} H_{d} b_{R}+M_{\eta} \bar{\eta}_{L} \eta_{R}+M_{\psi} \bar{\psi}_{L} \psi_{R},
$$

such that after integrating out the heavy fields the effective Lagrangian is given by

$$
\mathcal{L}_{E F T}=Y_{b}^{\mathrm{eff}} \bar{b}_{L} H_{d} b_{R}
$$

with

$$
Y_{b}^{\mathrm{eff}} \equiv \varepsilon y^{d}=\frac{y_{1} y_{2} y_{3}}{M_{\eta} M_{\psi}} \frac{v_{u} v_{d}}{2}
$$




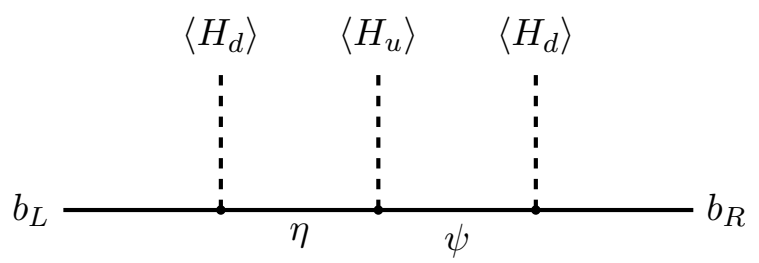

Figure 20. Diagram in the full theory, which generates the Yukawa coupling between the Higgs and the bottom quarks after integrating out the heavy vector-like fermions $\psi, \eta$.

The corresponding diagram is given in figure 20 in which the new vector-like fermions carry quantum numbers

$$
\eta_{L, R} \sim(\mathbf{3}, \mathbf{1},-1 / 3,2), \quad \psi_{L, R} \sim(\mathbf{3}, \mathbf{2}, 1 / 6,1)
$$

with respect to the groups $\left(\mathrm{SU}(3)_{C}, \mathrm{SU}(2)_{L}, \mathrm{U}(1)_{Y}, \mathrm{U}(1)_{F}\right)$.

From (8.3) is follows that for fixed $y_{1}=y_{2}=y_{3}=1$ and $y^{d} \in[0.5,1.5]$ this predicts the masses $M_{\eta}=M_{\psi} \approx \Lambda=1 \mathrm{TeV}$. It is evident that slightly larger fundamental Yukawa couplings $y_{1}, y_{2}$ and $y_{3}$, allow for heavier vector-like fermions, while any $\tan \beta \gg 1$ or $\tan \beta \ll 1$ lead to lower mass scales. In the spirit of avoiding hierarchies between the fundamental couplings, including the top Yukawa coupling, we shall consider the ratio $y_{i} / y_{t} \sim \mathcal{O}(1)$ with $i=1,2,3$. This constrains the masses of the vector-like fermions to be at most of the order of a few TeV. In particular, we define a generic mass $\bar{M} \equiv \sqrt{M_{\eta} M_{\psi}}$, and an average fundamental Yukawa coupling $\bar{y}=\left(y_{1} y_{2} y_{3}\right)^{1 / 3}$, such that

$$
\bar{M}^{2}=\frac{\bar{y}^{3}}{y^{d}} \frac{v^{2}}{2 \varepsilon} \frac{t_{\beta}}{1+t_{\beta}^{2}} .
$$

In figure 21, we show the expected masses of the new fermions for varying $\tan \beta$ and fixed $y^{d}=1$, for three different values of average Yukawa couplings $\bar{y}=1,1.5,2$ (from bottom to top). These predictions for the expected masses remain the same for $\bar{y}=1$ and change at most by $15 \%(25 \%)$ for $\bar{y}=1.5$ (2) for the first generation quarks and at most $10 \%(20 \%)$ for second generation quarks.

The solid and dashed red lines in figure 21 indicate the present and projected experimental bounds from searches for pair produced heavy quarks at the LHC. These searches have been performed both by ATLAS and CMS, and exclude vector resonances with masses of $600-800 \mathrm{GeV}$ [74-76], depending on the decay mode, with some channels already probing top partners $T$ up to $900 \mathrm{GeV}$ for $\operatorname{Br}\left(T \rightarrow W^{+} b\right)=100 \%$ [77]. The next run of the LHC has a projected reach of $\bar{M} \gtrsim 1.2(1.4) \mathrm{TeV}$ for $20 \mathrm{fb}^{-1}\left(100 \mathrm{fb}^{-1}\right)$ and $\operatorname{Br}\left(T \rightarrow W^{+} b\right)=50 \%$ [78]. Searches for heavy vector-like quarks in single production have also been considered [79-81] and could be much more effective as a discovery channel for sufficiently heavy vector-like quarks compared to the previously mentioned pair production searches. However, the LHC reach in the single production channel depends very strongly on the model parameters which define the couplings of the heavy quarks to SM quarks. A reinterpretation of any of the existing LHC bounds in single heavy quark production channels would demand a detailed study of production cross sections and decay branching 


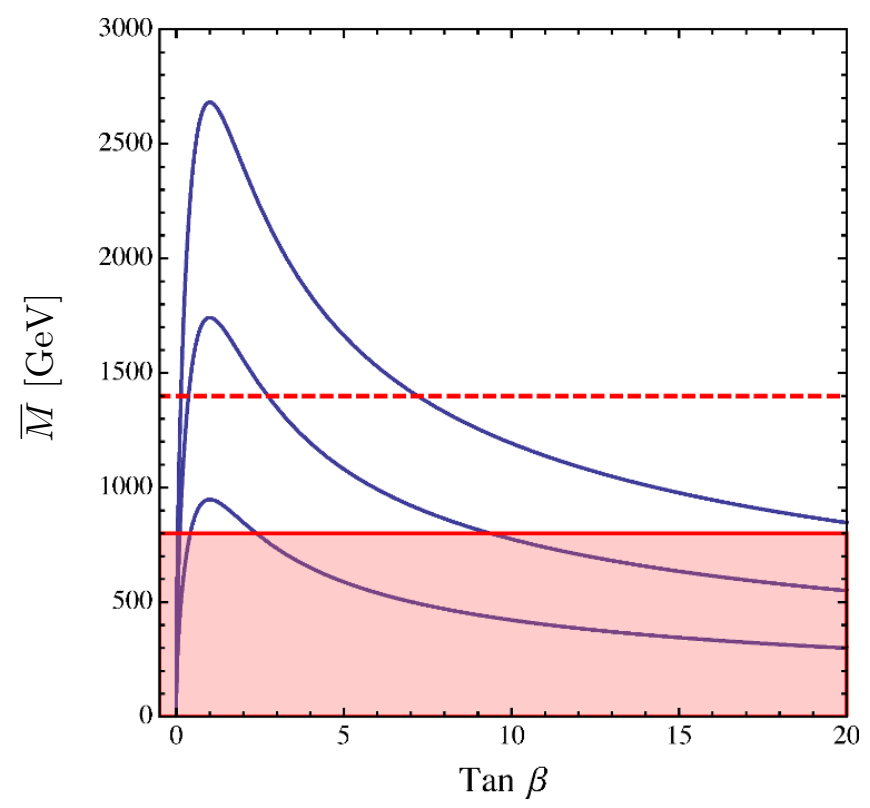

Figure 21. Masses of the new fermions in the UV completion depending on $\tan \beta$ and for three different values of the average Yukawa coupling $\bar{y}=1,1.5,2$ (from bottom to top). Fermion masses below the solid red line are excluded by current LHC bounds, while the dashed red line shows the expected exclusion reach for the $14 \mathrm{TeV}$ run of the LHC.

ratios for a specific UV completion. Similarly, a specific UV completion would be subject to constraints from electroweak precision measurements as well as from flavor physics [73]. The latter have been addressed in some detail in the original Giudice-Lebedev paper [3].

\section{$9 \quad$ Benchmark scenarios}

The global fit to Higgs signal strength measurements discussed in section 3 universally constrains the allowed parameter space to two branches within $\cos (\beta-\alpha)=0.35-0.8$. Smaller values of $\cos (\beta-\alpha)<0.35$ are in principle possible for $\tan \beta>5$, but such large values of $\tan \beta$ are in tension with flavor observables. Electroweak precision observables and collider searches for the extra scalars provide additional constraints that narrow the parameter space significantly. In the following we examine the allowed window in the $\cos (\beta-\alpha)-\tan \beta$ plane and specify three benchmark points, that highlight the interesting features for the phenomenology of this model, and for which we give a detailed list of couplings, production cross sections and decay widths.

As a result of the discussion in sections 6, the combination of constraints from flavor physics, electroweak precision observables, unitarity and perturbativity lead to a constrained region of mass values for the additional Higgs bosons $M_{A} \approx M_{H} \approx 500-600 \mathrm{GeV}$ and $M_{H^{+}} \approx 360-500 \mathrm{GeV}$. Perturbativity puts an upper bound of $600 \mathrm{GeV}$ on the neutral Higgs masses and requires a splitting between the neutral and charged Higgs masses of $M_{A, H}-M_{H^{+}} \gtrsim 100 \mathrm{GeV}$. Electroweak precision measurements exclude the left branch of the global fit to Higgs coupling measurements for values of $\tan \beta \lesssim 4.5$. In addition, tree-level contributions to meson-antimeson mixing put an upper bound of $\tan \beta \lesssim 5$, while 
loop contributions from charged Higgs exchange result in a lower bound of $\tan \beta \gtrsim 1.5$. Collider searches for the two heavy neutral Higgs bosons further constrain the allowed parameter space and probe the right branch of the global Higgs fit for $\cos (\beta-\alpha)=\mathcal{O}(0.5)$ and $\tan \beta \lesssim 3$. As a result, there is a specific window of allowed masses as well as values of $\cos (\beta-\alpha)$ and $\tan \beta$, which translates into a precise prediction for searches for the extra scalars and constrain the possible deviations in the SM Higgs couplings. In figure 22, we illustrate this window by showing the 95\% CL region of the global fit to ATLAS Higgs signal strengths measurements (red shaded area), the region preferred by electroweak precision constraints (shaded green) and the bound induced from flavor constraints (solid purple contour), as shown in figure 9. Further, we superimpose the bounds derived from the ATLAS and CMS measurements of $\sigma(g g \rightarrow A) \times \operatorname{Br}\left(A \rightarrow h Z \rightarrow b \bar{b} \ell^{+} \ell^{-}\right)$(solid blue) and $\sigma(p p \rightarrow H+X) \times \operatorname{Br}(H \rightarrow V V)$ (solid orange). In the left panel we assume scalar masses of $M_{H}=M_{A}=600 \mathrm{GeV}, M_{H^{+}}=450 \mathrm{GeV}$, and in the right panel $M_{H}=M_{A}=500 \mathrm{GeV}$, $M_{H^{+}}=360 \mathrm{GeV}$. The gray shaded area is excluded, the overlap of the light green and red regions is allowed.

Comparing the two plots in figure 22, bounds from flavor physics as well as collider constraints become weaker for larger masses. The area in agreement with electroweak precision bounds is slightly larger for smaller mass splittings, but similar for the two examples given in figure 22. The right boundary of the right branch of the global Higgs fit is close to the contour of $\kappa_{b}=-1$, for which the Higgs coupling to bottom quarks has the same size, but opposite sign compared to the SM one. The left boundary of the right branch is close to $\kappa_{b}=-0.5$. For all of the allowed parameter space, we can therefore infer $-1 \lesssim \kappa_{b} \lesssim-0.5$. In addition to the sign and the reduction of the Higgs bottom coupling, we find a universal enhancement of the Higgs charm couplings. Both can in principle be probed by measurements of exclusive radiative Higgs boson decays [82]. The $14 \mathrm{TeV}$ run of the LHC can potentially test the sign of $\kappa_{b}$, whereas possible departures from the SM Higgs charm couplings of the order of $20 \%$ could be established at a prospective $100 \mathrm{TeV}$ collider by measurements of these radiative hadronic modes [83]. A first experimental analysis of the decays $h \rightarrow J / \psi \gamma$ and $h \rightarrow \Upsilon \gamma$, including the relevant backgrounds, has recently been performed by ATLAS [84]. Less optimistic limits for the charm Yukawa coupling in the exclusive decays have been obtained by the authors of [85], who however find a comparable sensitivity in measurements of the inclusive decay rate into charms using charm tagging techniques [86]. Moreover, in the presence of a Higgs portal to dark matter, relative signs in the Higgs couplings to light and top quarks could significantly modify the direct detection cross section [87].

In table 4 and 5 we give the values for the Higgs couplings, signal strengths, production cross sections and branching ratios for three representative benchmark points indicated by black crosses in figure 22 . Typical values of $\cos (\beta-\alpha) \approx 0.4-0.55$ and $\tan \beta \approx 3-4.5$ are considered. In all cases, $\kappa_{t} \approx 1$, implying a gluon fusion production rate of order of the SM one.

Benchmarks 1a and $\mathbf{1 b}$ allow for larger values $M_{A, H} \approx 600 \mathrm{GeV}$ and a charged Higgs mass $M_{H^{+}} \approx 450 \mathrm{GeV}$, close to the $2 \sigma$ bound derived from the experimental $b \rightarrow s \gamma$ measurement in a type II two Higgs doublet model with $\tan \beta>2$. 
Benchmark 1: $M_{A}=M_{H}=600 \mathrm{GeV}, M_{H^{+}}=450 \mathrm{GeV}$,

$$
\begin{aligned}
& \text { 1a } \cos (\beta-\alpha)=0.55, \quad \tan \beta=3, \\
& \text { 1b } \cos (\beta-\alpha)=0.42, \quad \tan \beta=4.5,
\end{aligned}
$$

Light Higgs Couplings:

$1 \mathrm{a} \quad \kappa_{t}=1.02, \quad \kappa_{V}=0.84, \quad \kappa_{b}=\kappa_{\tau}=-0.61, \quad \kappa_{c}=1.22, \quad \kappa_{s}=-0.41$,

1b $\kappa_{t}=1.00, \quad \kappa_{V}=0.91, \quad \kappa_{b}=\kappa_{\tau}=-0.96, \quad \kappa_{c}=1.02, \quad \kappa_{s}=-0.95$,

Higgs Signal Strength:

\begin{tabular}{|l|c|c|c|c|}
\hline $\mathbf{1 a}$ & $\mu_{V}$ & $\mu_{\gamma}$ & $\mu_{b}$ & $\mu_{c}$ \\
\hline$\sigma_{g g \rightarrow h}$ & 1.38 & 1.21 & 0.74 & 2.95 \\
\hline$\sigma_{t \bar{t} \rightarrow h}$ & 1.33 & 1.17 & 0.71 & 2.84 \\
\hline$\sigma_{V B F}, \sigma_{V H}$ & 0.89 & 0.78 & 0.48 & 1.91 \\
\hline
\end{tabular}

\begin{tabular}{|l|c|c|c|c|}
\hline $\mathbf{1 b}$ & $\mu_{V}$ & $\mu_{\gamma}$ & $\mu_{b}$ & $\mu_{c}$ \\
\hline$\sigma_{g g \rightarrow h}$ & 0.96 & 0.91 & 1.09 & 1.22 \\
\hline$\sigma_{t \bar{t} \rightarrow h}$ & 0.90 & 0.85 & 1.02 & 1.14 \\
\hline$\sigma_{V B F}, \sigma_{V H}$ & 0.74 & 0.70 & 0.84 & 0.94 \\
\hline
\end{tabular}

Heavy Scalar Production Cross sections for 1a (1b):

$8 \mathrm{TeV}: \quad \sigma(g g \rightarrow A)=78(36) \mathrm{fb}, \quad \sigma(g g \rightarrow H)=32(21) \mathrm{fb}$,

$$
\sigma\left(p p \rightarrow H^{-} t(b)\right)=9(4) \mathrm{fb},
$$

14 TeV: $\sigma(g g \rightarrow A)=361(157) \mathrm{fb}, \quad \sigma(g g \rightarrow H)=166(97) \mathrm{fb}$,

$$
\sigma\left(p p \rightarrow H^{-} t(b)\right)=63(25) \mathrm{fb},
$$

Heavy Scalar Decay Modes:

\begin{tabular}{|c|c|c|}
\hline$A$ & \multicolumn{2}{|c|}{$\Gamma_{i} / \Gamma_{A}$} \\
\hline & $\mathbf{1 a}$ & $\mathbf{1 b}$ \\
\hline$Z h$ & $70.2 \%$ & $62 \%$ \\
$W^{-} H^{+}$ & $14.4 \%$ & $21.8 \%$ \\
$b \bar{b}$ & $1.6 \%$ & $5.2 \%$ \\
$t \bar{t}$ & $12.9 \%$ & $8.7 \%$ \\
$\tau^{+} \tau^{-}$ & $0.2 \%$ & $0.7 \%$ \\
$t \bar{c}$ & $0.4 \%$ & $1.1 \%$ \\
\hline
\end{tabular}

\begin{tabular}{|c|c|c|}
\hline$H$ & \multicolumn{2}{|c|}{$\Gamma_{i} / \Gamma_{H}$} \\
\hline & $\mathbf{1 a}$ & $\mathbf{1 b}$ \\
\hline$W W$ & $52.9 \%$ & $43 \%$ \\
$Z Z$ & $25.6 \%$ & $20.9 \%$ \\
$h h$ & $9.2 \%$ & $16.9 \%$ \\
$W^{-} H^{+}$ & $6.8 \%$ & $11.2 \%$ \\
$t \bar{t}$ & $3.9 \%$ & $3.5 \%$ \\
\hline
\end{tabular}

\begin{tabular}{|c|c|c|}
\hline$H^{+}$ & \multicolumn{2}{|c|}{$\Gamma_{i} / \Gamma_{H^{+}}$} \\
\hline & $\mathbf{1 a}$ & $\mathbf{1 b}$ \\
\hline$h W$ & $78.7 \%$ & $81.5 \%$ \\
$t \bar{b}$ & $21.2 \%$ & $18.2 \%$ \\
$\tau \nu$ & $0.048 \%$ & $0.33 \%$ \\
\hline
\end{tabular}

Total Width for 1a (1b):

$\Gamma_{h}=2.22(3.71) \mathrm{MeV}, \quad \Gamma_{A}=24.6(16.3) \mathrm{GeV}, \quad \Gamma_{H}=36.4(26.1) \mathrm{GeV}$,

$\Gamma_{H^{+}}=10.2(5.8) \mathrm{GeV}$.

Table 4. Values for the Higgs signal strength, heavy scalar production cross sections for the dominant channels at the LHC, partial and total widths for the benchmarks $\mathbf{1 a}$ and $\mathbf{1} \mathbf{b}$. 
Benchmark 2: $M_{A}=M_{H}=500 \mathrm{GeV}, M_{H^{+}}=360 \mathrm{GeV}$, $\cos (\beta-\alpha)=0.45, \quad \tan \beta=4$,

Light Higgs Couplings:

1b $\kappa_{t}=1.01, \quad \kappa_{V}=0.9, \quad \kappa_{b}=\kappa_{\tau}=-0.81, \quad \kappa_{c}=1.1, \quad \kappa_{s}=-0.71$,

Higgs Signal Strength:

\begin{tabular}{|l|c|c|c|c|}
\hline $\mathbf{2}$ & $\mu_{V}$ & $\mu_{\gamma}$ & $\mu_{b}$ & $\mu_{c}$ \\
\hline$\sigma_{g g \rightarrow h}$ & 1.15 & 1.07 & 0.94 & 1.76 \\
\hline$\sigma_{t \bar{t} \rightarrow h}$ & 1.09 & 1.02 & 0.90 & 1.67 \\
\hline$\sigma_{V B F}, \sigma_{V H}$ & 0.86 & 0.80 & 0.71 & 1.32 \\
\hline
\end{tabular}

Heavy Scalar Production Cross sections:

$8 \mathrm{TeV}: \quad \sigma(g g \rightarrow A)=130 \mathrm{fb}, \quad \sigma(g g \rightarrow H)=53 \mathrm{fb}, \quad \sigma\left(p p \rightarrow H^{-} t(b)\right)=12 \mathrm{fb}$, $14 \mathrm{TeV}: \sigma(g g \rightarrow A)=546 \mathrm{fb}, \quad \sigma(g g \rightarrow H)=224 \mathrm{fb}, \quad \sigma\left(p p \rightarrow H^{-} t(b)\right)=66 \mathrm{fb}$, Heavy Scalar Decay Modes:

\begin{tabular}{|c|c|}
\hline$A$ & $\Gamma_{i} / \Gamma_{A}$ \\
\hline$Z h$ & $56.6 \%$ \\
$W^{-} H^{+}$ & $23.3 \%$ \\
$b \bar{b}$ & $5.3 \%$ \\
$t \bar{t}$ & $12.4 \%$ \\
$\tau^{+} \tau^{-}$ & $0.66 \%$ \\
$t \bar{c}$ & $1.1 \%$ \\
\hline
\end{tabular}

\begin{tabular}{|c|c|}
\hline$H$ & $\Gamma_{i} / \Gamma_{H}$ \\
\hline$W W$ & $45.4 \%$ \\
$Z Z$ & $21.8 \%$ \\
$h h$ & $11.5 \%$ \\
$W^{-} H^{+}$ & $12.6 \%$ \\
$t \bar{t}$ & $3.65 \%$ \\
\hline
\end{tabular}

\begin{tabular}{|c|c|}
\hline$H^{+}$ & $\Gamma_{i} / \Gamma_{H^{+}}$ \\
\hline$h W$ & $71.8 \%$ \\
$t \bar{b}$ & $27.8 \%$ \\
$\tau \nu$ & $0.4 \%$ \\
\hline
\end{tabular}

Total Width:

$\Gamma_{h}=3 \mathrm{MeV}, \quad \Gamma_{A}=10.7 \mathrm{GeV}, \quad \Gamma_{H}=15.7 \mathrm{GeV}, \quad \Gamma_{H^{+}}=3 \mathrm{GeV}$.

Table 5. Values for the Higgs signal strength, heavy scalar production cross sections for the dominant channels at the LHC, partial and total widths for the benchmark $\mathbf{2}$.

In Benchmark 1a, the tree-level gauge boson and down type fermion third generation couplings are suppressed by factors of order $20 \%$ and $40 \%$, respectively, while the Higgs coupling to charm is enhanced by about $20 \%$. The sizable suppression of $\kappa_{b}$ yields a suppression of the branching ratio into gauge bosons and hence of the corresponding signal strength of those channels. The charm signal strength instead, is increased by a factor $\sim 2-3$ (depending on the production mode) due to the combined effects of an enhancement in $\kappa_{c}$ and a suppression in $\kappa_{b}$ and $\kappa_{V}$. All other vector boson fusion and $\mathrm{VH}$ production channels are suppressed with respect to the SM, in particular the $h \rightarrow b \bar{b}$ search mode.

In Benchmark 1b all tree-level fermion and gauge Higgs couplings are within less than $5-10 \%$ of the SM expectations, hence the signal strengths in gluon fusion production are also within $5-10 \%$ of the SM ones, with the exception of a $20 \%$ enhancement in $\mu_{c}$. All 
vector boson fusion/VH production channels are suppressed with a maximal suppression of $25-30 \%$ in the case of light Higgs decaying into gauge bosons.

Benchmark 2 allows for the smallest possible values of $M_{H^{+}}=360 \mathrm{GeV}$ compatible with the $3 \sigma$ bounds derived from the experimental $b \rightarrow s \gamma$ measurement in a type II 2HDM with $\tan \beta>2$. Benchmark 2 has a similar tendency in the couplings of gauge bosons and fermions to the light Higgs boson and hence in the corresponding signal strengths as in Benchmark 1a, but with percentual effects in the deviations from SM predictions that are a factor 2-3 smaller. In addition to improving signal strength measurements, the ongoing run of the LHC will probe these benchmarks by direct searches for the additional Higgs bosons. All three benchmark scenarios will be primarily tested by the search for $A \rightarrow Z h$ and $H \rightarrow V V$, that have branching ratios of $55 \%-75 \%$, depending on the scenario. In the case of $H \rightarrow V V$, the inclusive and vector boson fusion production modes will play a complementary, relevant role. In addition to these discovery channels, other interesting search modes such as $A, H \rightarrow W^{+} H^{-}, H \rightarrow h h, A \rightarrow t \bar{t}, H^{+} \rightarrow h W^{+}$, and $H^{+} \rightarrow t \bar{b}$ would yield additional valuable information about this model. The mass splitting between neutral and charged scalars give rise to an additional decay chain, that can potentially allow to discover the charged Higgs even for masses of $M_{H^{+}} \approx 360-400 \mathrm{GeV}$, in particular for the subsequent decay of $H^{+} \rightarrow W^{+} h$. Although challenging due to the small branching ratio, a novel channel in these scenarios is $A \rightarrow t \bar{c}$.

Predictions for particular observables can be computed from the information provided in table 4 and table 5. Finite width effects play a relevant role and in the case of $A \rightarrow h Z$ we have compiled them in the right panel in figure 16. For completeness, we present the branching ratios for $h, H, A$ and $H^{+}$as a function of $\cos (\beta-\alpha)$ for the parameter values of benchmark 1a, $1 \mathrm{~b}$ and 2 in figures 23, 24 and 25 in appendix E.

Finally, improved measurements of flavor observables, in particular in the neutral $B_{d}$ system could additionally constrain the parameter space significantly.

\section{Conclusion}

In this article we propose an explanation for the hierarchies in fermion masses and mixings based on a Froggatt-Nielsen mechanism, in which two Higgs doublets play the role of the flavon. Therefore, the underlying flavor symmetry is broken at the electroweak scale. The flavor charges are fixed to reproduce the SM quark mass hierarchies and CKM mixing angles up to rescalings, that have no effect on any physical quantity. As a result, this two Higgs doublet flavor model can be described by few effective parameters, the masses of the extra scalars $M_{H}, M_{A}, M_{H^{+}}, \cos (\beta-\alpha)$ and $\tan \beta$. This allows us to present our main findings in the $\cos (\beta-\alpha)-\tan \beta$ plane for fixed mass values, as shown in figure 22 .

Modified interactions between the SM-like Higgs $h$ and quarks are characteristic for our two Higgs doublet flavor model, leading to strong constraints from Higgs signal strength measurements. The results of our Higgs global fit to ATLAS and CMS data constrain possible deviations of the couplings of the light Higgs to fermions and gauge bosons with respect to the $\mathrm{SM}$ ones, and select sizable values of $\cos (\beta-\alpha) \approx \mathcal{O}(0.5)$. This implies a suppression of the tree-level couplings of the Higgs to gauge bosons, which is proportional to 

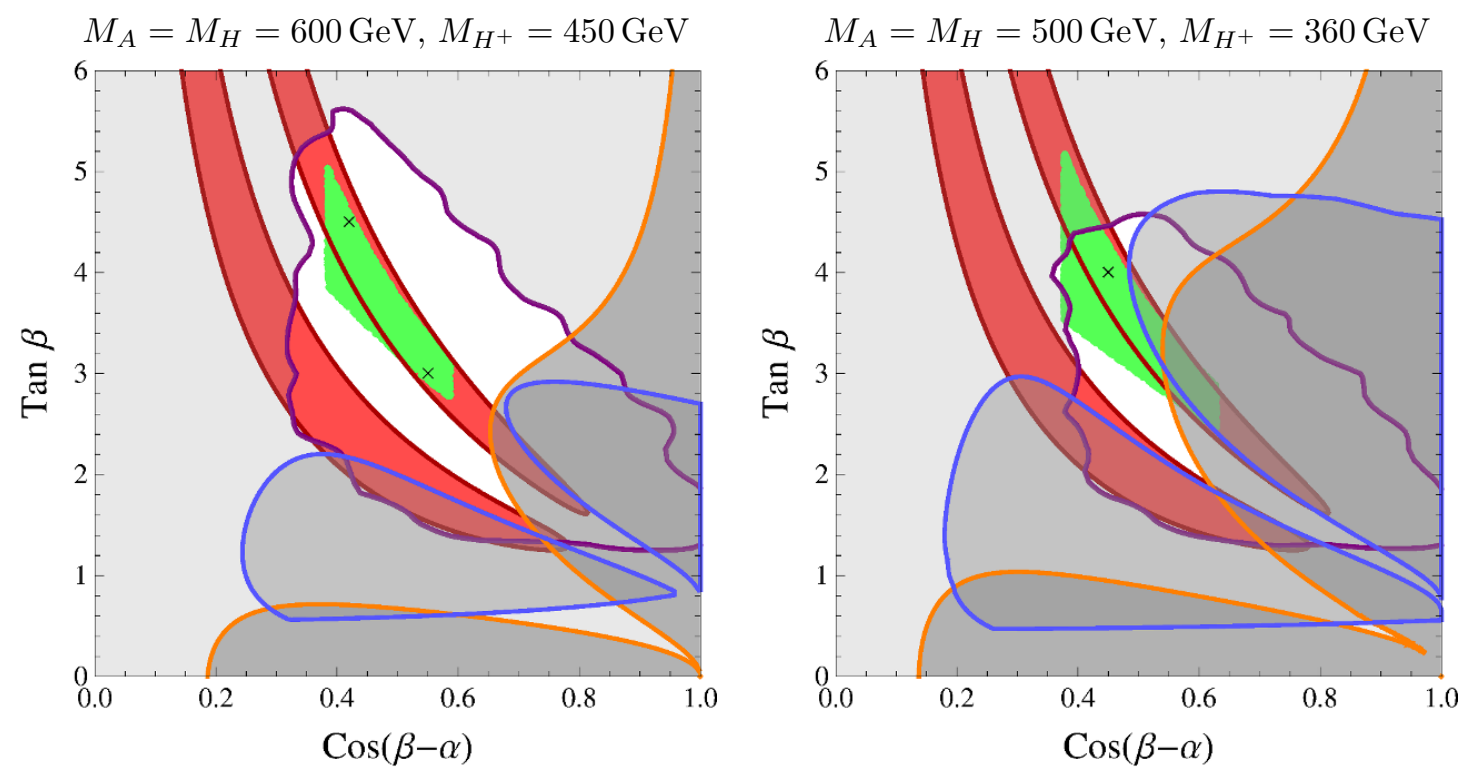

Figure 22. Summary plots showing constraints from flavor observables (purple contour) and direct collider searches for $A \rightarrow h Z \rightarrow \ell^{+} \ell^{-} b \bar{b}$ (blue contour) as well as $H \rightarrow W^{+} W^{-} / Z Z$ (orange contour), where the gray shaded area shows exclusion. The red shaded region is allowed at the 95\% CL from the global fit to Higgs signal strength measurements to ATLAS data. The green area highlights the allowed region from electroweak precision observables, perturbativity and unitarity constraints. The panels correspond to $M_{A}=M_{H}=600 \mathrm{GeV}$ and $M_{H^{+}}=450 \mathrm{GeV}$ (left), and $M_{A}=M_{H}=500 \mathrm{GeV}$ and $M_{H^{+}}=360 \mathrm{GeV}$ (right). The black crosses in both panels indicate the benchmark scenarios.

$\sin (\beta-\alpha)$ as in any two Higgs doublet model and therefore a suppressed vector boson fusion production rate with respect to the SM. The alignment/decoupling limit $\cos (\beta-\alpha)=0$ is excluded for all values of $\tan \beta$, since in this limit our model approaches the Babu-NandiGiudice-Lebedev model for which there is a factor of three enhancement for the coupling of the light Higgs to bottom quarks. The Higgs global fit allows for two branches in the $\cos (\beta-\alpha)-\tan \beta$ plane (red shaded areas in figure 22) with opposite sign of the bottom Yukawa coupling. However, other constraints end up singling out the branch with values of the SM normalized light Higgs-bottom Yukawa coupling between -0.5 and -1 . On this branch the light Higgs-top Yukawa coupling is close to its SM value, implying gluon fusion signal strengths of $\mathcal{O}(1)$. Furthermore, on this branch, the coupling of the light Higgs to charm quarks is universally enhanced by up to $30 \%$, leading to a possible enhancement of the Higgs to charm signal strength by a factor of three. Both the negative sign of the bottom Higgs coupling as well as the enhanced Higgs to charm signal strength can in principle be measured at a high luminosity/energy collider through exclusive Higgs decays with a final state photon, such as $h \rightarrow \Upsilon \gamma$ and $h \rightarrow J / \psi \gamma$.

Flavor changing neutral currents arise at tree-level, mediated by the light Higgs as well as the extra neutral scalars. Remarkably, light Higgs FCNCs become automatically small for the branch of the global Higgs fit with negative light Higgs-bottom Yukawa coupling. While the masses of the extra neutral scalars are constrained to be larger than $500 \mathrm{GeV}$ in 
this region, we need a mild fine-tuning of $\mathcal{O}(10 \%)$ in the Yukawa couplings in order not to exceed the strongest constraint from $B_{d}-\bar{B}_{d}$ mixing (shown as purple contour in figure 22). These tree-level FCNCs result in an upper bound of $\tan \beta \lesssim 5.5$. Moreover, contributions from box diagrams with charged Higgs exchange can compete with the tree-level diagrams for low $\tan \beta$ and exclude values of $\tan \beta \lesssim 1$. Thus the interplay of tree-level and loop contributions in flavor observables predicts $5.5 \gtrsim \tan \beta \gtrsim 1$. Interestingly, if we discard the explanation of the CKM angles by the two Higgs doublet flavor model, we find almost no constraints from flavor observables in the region preferred by the global Higgs fit. As in any two Higgs doublet model, charged Higgs exchanges also induce FCNCs through penguin diagrams, for example $b \rightarrow s \gamma$, which imply a lower bound on the charged Higgs mass of $360 \mathrm{GeV}$ for $\tan \beta \gtrsim 2$.

The two Higgs doublet flavor model offers exciting possibilities for direct collider searches for the additional Higgs bosons. Electroweak precision observables, perturbativity and unitarity constraints choose a preferred range of masses and mass splittings for the new heavy scalars. In particular, almost degenerate values for the CP-odd and CP-even Higgs boson masses and sizable splitting between the neutral and charged Higgs masses are strongly favoured. This opens the opportunity of new decay channels, $A \rightarrow H^{+} W^{-}$ and $H \rightarrow H^{+} W^{-}$in addition to the regular decay channels $H \rightarrow W^{+} W^{-} / Z Z, A \rightarrow h Z$, that are importantly enhanced in the $\cos (\beta-\alpha) \approx \mathcal{O}(0.5)$ region. The latter are the leading discovery modes for these scalars (present bounds are shown by blue and orange contours in figure 22). Furthermore, the $\cos (\beta-\alpha)$ dependence of the $H W^{+} W^{-}, H Z Z$ couplings are of particular relevance because the vector boson fusion production mode can compensate for the suppression of the gluon fusion production mode of the CP even Higgs in the relevant regions of parameter space. Direct searches for a charged Higgs boson are not sensitive for masses compatible with the flavor constraints, however future searches via Higgs decay chains with the subsequent decay $H^{+} \rightarrow W^{+} h$ may be promising. The other possible decay of heavy Higgs bosons to the SM Higgs is in the channel $H \rightarrow h h$ with branching ratios of order $10 \%$.

The fact that the flavor symmetry is broken at the electroweak scale predicts a UV completion in the few $\mathrm{TeV}$ range, as well as a low value of $\tan \beta$ in agreement with flavor constraints. The necessity of new physics at the $\mathrm{TeV}$ scale provides an additional motivation for the search for new vector-like fermions at the run II of LHC.

We conclude, that in the two Higgs flavor model constraints from flavor observables, Higgs precision measurements, direct heavy Higgs searches, and precision electroweak observables, as well as unitarity and perturbativity restrictions on the theory, can be fulfilled simultaneously. We propose three benchmark scenarios in this region, that highlight different characteristics of the two Higgs doublet flavor model (black crosses in figure 22). In table 4 and 5 we provide all the relevant information to compute production cross sections and decay rates for these benchmark scenarios and test the two Higgs doublet flavor model at the run II of LHC. 


\section{Acknowledgments}

We thank Prateek Agrawal, Wolfgang Altmannshofer, Andrzej Buras, Thorsten Feldmann, Elisabetta Furlan, Joerg Jaeckel, Matthias Neubert, Tilman Plehn, Raoul Röntsch and Carlos Wagner for useful comments and discussions. We specially thank Zhen Liu for very helpful comments about the Higgs boson phenomenology and Mikolaj Misiak for private discussions on the bound of charged Higgs masses from $\operatorname{Br}\left(B_{s} \rightarrow X_{s} \gamma\right)$ in two Higgs doublet models. KG was supported by the Deutsche Forschungsgemeinschaft (DFG), grant number GE 2541/1-1. MB acknowledges the support of the Alexander von Humboldt Foundation. Fermilab is operated by Fermi Research Alliance, LLC under Contract No. DE-AC0207CH11359 with the United States Department of Energy.

\section{A The Higgs potential}

In this appendix we consider the scalar potential and related topics.

The fact that $H_{u} H_{d}$ carries a flavor charge strongly constrains the scalar potential. We need a (soft) source of flavor breaking in order to generate a $b$-term. We consider this additional source of flavor breaking to be irrelevant for the texture of the Yukawa couplings.

The potential reads then

$$
\begin{aligned}
V\left(H_{u}, H_{d}\right)= & \mu_{u}^{2} H_{u}^{\dagger} H_{u}+\mu_{d}^{2} H_{d}^{\dagger} H_{d}-\left[b H_{u} H_{d}+h . c .\right] \\
& +\frac{\lambda_{1}}{2}\left(H_{u}^{\dagger} H_{u}\right)^{2}+\frac{\lambda_{2}}{2}\left(H_{d}^{\dagger} H_{d}\right)^{2}+\lambda_{3}\left(H_{u}^{\dagger} H_{u}\right)\left(H_{d}^{\dagger} H_{d}\right)+\lambda_{4}\left(H_{u}^{\dagger} H_{d}\right)\left(H_{d}^{\dagger} H_{u}\right)
\end{aligned}
$$

in which $H_{u} H_{d} \equiv H_{u}^{T}\left(i \sigma_{2}\right) H_{d}$. Note that the potential is the same as in a generic CPconserving two Higgs doublet model, see for example [9, 12], whith $\lambda_{5}=\lambda_{6}=\lambda_{7}=0$.

In order to diagonalize the potential, we introduce the neutral scalar mass eigenstates,

$$
\left(\begin{array}{c}
h \\
H
\end{array}\right)=\left(\begin{array}{cc}
c_{\alpha} & -s_{\alpha} \\
s_{\alpha} & c_{\alpha}
\end{array}\right)\left(\begin{array}{l}
\operatorname{Re} H_{u}^{0} \\
\operatorname{Re} H_{d}^{0}
\end{array}\right),
$$

with the mixing angles $c_{\alpha}=\cos \alpha$ and $s_{\alpha}=\sin \alpha$ as well as the pseudoscalar mass eigenstates

$$
\left(\begin{array}{c}
\pi^{0} \\
A
\end{array}\right)=\left(\begin{array}{cc}
s_{\beta} & -c_{\beta} \\
c_{\beta} & s_{\beta}
\end{array}\right)\left(\begin{array}{l}
\operatorname{Im} H_{u}^{0} \\
\operatorname{Im} H_{d}^{0}
\end{array}\right),
$$

and similarly for the charged mass eigenstates $H^{ \pm}$,

$$
\left(\begin{array}{c}
\pi^{-} \\
H^{-}
\end{array}\right)=\left(\begin{array}{cc}
s_{\beta} & -c_{\beta} \\
c_{\beta} & s_{\beta}
\end{array}\right)\left(\begin{array}{c}
H_{u}^{-} \\
H_{d}^{+*}
\end{array}\right) .
$$

Performing these rotations the explicit formulas for masses of scalar fields can be obtained, see for details for example $[9,12]$.

Finally from the scalar potential we obtain all couplings between the scalars [5, 9, 12]. Particularly relevant for our analysis is the coupling between the heavy scalar $H$ and the 
light Higgs $h$ given in equation (7.4), and the coupling of the light Higgs $h$ to two charged Higgs bosons $H^{ \pm}$

$$
g_{h H^{+} H^{-}}=\frac{1}{v}\left[\left(2 M_{A}^{2}-2 M_{H^{ \pm}}^{2}-m_{h}^{2}\right) s_{\beta-\alpha}+2\left(M_{A}^{2}-m_{h}^{2}\right) \frac{c_{2 \beta} c_{\beta-\alpha}}{s_{2 \beta}}\right] .
$$

\section{B Box diagrams and loop functions}

In this appendix, we collect the contributions to the Wilson coefficients (5.1) from box diagrams and the relevant loop functions [37, 42, 88]. For $K-\bar{K}$ mixing we have the following Wilson coefficients:

$$
C_{1, \mathrm{box}}^{s d}=\frac{G_{F}^{2} m_{W}^{2}}{16 \pi^{2}}\left(\left(\lambda_{s d}^{t}\right)^{2} C_{1, \mathrm{box}}^{t}+\left(\lambda_{s d}^{c}\right)^{2} C_{1, \mathrm{box}}^{c}+2 \lambda_{s d}^{t} \lambda_{s d}^{c} C_{1, \mathrm{box}}^{c t}\right)
$$

with $\lambda_{t}=V_{t d} V_{t s}^{*}, \lambda_{c}=V_{c d} V_{c s}^{*}$ and

$$
\begin{aligned}
C_{1, \text { box }}^{t}= & \left(4 x_{t}+x_{t}^{2}\right) m_{W}^{2} D_{2}\left(m_{t}, m_{W}\right)-8 x_{t}^{2} m_{W}^{4} D_{0}\left(m_{t}, m_{W}\right) \\
& +\frac{2 x_{t}^{2}}{t_{\beta}^{2}}\left[m_{W}^{2} D_{2}\left(m_{t}, m_{W}, M_{H^{ \pm}}\right)-4 m_{W}^{4} D_{0}\left(m_{t}, m_{W}, M_{H^{ \pm}}\right)\right] \\
& +\frac{x_{t}^{2}}{t_{\beta}^{4}} m_{W}^{2} D_{2}\left(m_{t}, M_{H^{ \pm}}\right), \\
C_{1, \text { box }}^{c}= & \left(4 x_{c}+x_{c}^{2}\right) m_{W}^{2} D_{2}\left(m_{c}, m_{W}\right)-8 x_{c}^{2} m_{W}^{4} D_{0}\left(m_{c}, m_{W}\right), \\
C_{1, \text { box }}^{c t}= & \left(4 x_{c t}+x_{c t}^{2}\right) m_{W}^{2} D_{2}\left(m_{c}, m_{t}, m_{W}\right)-8 x_{c t}^{2} m_{W}^{4} D_{0}\left(m_{c}, m_{t}, m_{W}\right) \\
& +\frac{2 x_{c t}^{2}}{t_{\beta}^{2}}\left[m_{W}^{2} D_{2}\left(m_{c}, m_{t}, m_{W}, M_{H^{ \pm}}\right)-4 m_{W}^{4} D_{0}\left(m_{c}, m_{t}, m_{W}, M_{H^{ \pm}}\right)\right] \\
& +\frac{x_{c t}^{2}}{t_{\beta}^{4}} m_{W}^{2} D_{2}\left(m_{c} m_{t}, M_{H^{ \pm}}\right),
\end{aligned}
$$

in which $x_{t}=m_{t}^{2} / m_{W}^{2}, x_{c}=m_{c}^{2} / m_{W}^{2}$ and $x_{c t}=m_{c} m_{t} / m_{W}^{2}$. For $B_{d, s}-\bar{B}_{d, s}$ mixing, we have

$$
\begin{array}{rlrl}
C_{1, \mathrm{box}}^{b q} & =\frac{G_{F}^{2} m_{W}^{2}}{16 \pi^{2}}\left(\lambda_{b q}^{t}\right)^{2} C_{1, \mathrm{box}}^{t}, & \tilde{C}_{1, \mathrm{box}}^{b q}=\frac{G_{F}^{2} m_{W}^{2}}{16 \pi^{2}} \frac{m_{q}^{2} m_{b}^{2}}{m_{W}^{4}}\left(\lambda_{b q}^{t}\right)^{2} \tilde{C}_{1, \mathrm{box}}, \\
C_{2, \mathrm{box}}^{b q}=\frac{G_{F}^{2} m_{W}^{2}}{16 \pi^{2}} \frac{4 m_{q}^{2}}{m_{W}^{2}}\left(\lambda_{b q}^{t}\right)^{2} C_{2, \mathrm{box}}, & \tilde{C}_{2, \mathrm{box}}^{b q}=\frac{G_{F}^{2} m_{W}^{2}}{16 \pi^{2}} \frac{4 m_{b}^{2}}{m_{W}^{2}}\left(\lambda_{b q}^{t}\right)^{2} C_{2, \mathrm{box}}, \\
C_{4, \mathrm{box}}^{b q}=\frac{G_{F}^{2} m_{W}^{2}}{16 \pi^{2}} \frac{2 m_{q} m_{b}}{m_{W}^{2}}\left(\lambda_{b q}^{t}\right)^{2} C_{4, \mathrm{box}}, & C_{5, \mathrm{box}}^{b q}=\frac{G_{F}^{2} m_{W}^{2}}{16 \pi^{2}} \frac{m_{q} m_{b}}{m_{W}^{2}}\left(\lambda_{b q}^{t}\right)^{2} C_{5, \mathrm{box}},
\end{array}
$$


with $\lambda_{t}=V_{t b}^{*} V_{t q}$ and $(q=s, d)$ and

$$
\begin{aligned}
C_{1, \mathrm{box}}= & m_{W}^{2}\left[t_{\beta}^{4} \frac{m_{t}^{2}}{M_{H^{ \pm}}^{2}} D_{2}\left(m_{t}, M_{H^{ \pm}}\right)+t_{\beta}^{2} \bar{D}_{2}\left(m_{t}, m_{W}, M_{H^{ \pm}}\right)\right] \\
C_{2, \mathrm{box}}= & x_{t}^{2} m_{W}^{4}\left[D_{0}\left(m_{t}, M_{H^{ \pm}}\right)+2 D_{0}\left(m_{t}, m_{W}, M_{H^{ \pm}}\right)\right] \\
C_{4, \mathrm{box}}= & x_{t}^{2} m_{W}^{4}\left[4 D_{0}\left(m_{t}, M_{H^{ \pm}}\right)+\left(t_{\beta}^{2}+\frac{1}{t_{\beta}^{2}}\right) D_{2}\left(m_{t}, m_{W}, M_{H^{ \pm}}\right)\right] \\
& -4 t_{\beta}^{2} x_{t} m_{W}^{2} \bar{D}_{2}\left(m_{t}, m_{W}, M_{H^{ \pm}}\right), \\
C_{5, \mathrm{box}}= & x_{t}^{2} m_{W}^{2}\left[D_{2}\left(m_{t}, M_{H^{ \pm}}\right)+2 D_{2}\left(m_{t}, m_{W}, M_{H^{ \pm}}\right)\right] .
\end{aligned}
$$

The loop functions are given by

$$
\begin{aligned}
D_{0}\left(m_{1}, m_{2}, M_{1}, M_{2}\right)= & \frac{m_{2}^{2} \log \left(\frac{m_{2}^{2}}{m_{1}^{2}}\right)}{\left(m_{2}^{2}-m_{1}^{2}\right)\left(m_{2}^{2}-M_{1}^{2}\right)\left(m_{2}^{2}-M_{2}^{2}\right)} \\
& +\frac{M_{1}^{2} \log \left(\frac{M_{1}^{2}}{m_{1}^{2}}\right)}{\left(M_{1}^{2}-m_{1}^{2}\right)\left(M_{1}^{2}-m_{2}^{2}\right)\left(M_{1}^{2}-M_{2}^{2}\right)} \\
& +\frac{M_{2}^{2} \log \left(\frac{M_{2}^{2}}{m_{1}^{2}}\right)}{\left(M_{2}^{2}-m_{1}^{2}\right)\left(M_{2}^{2}-m_{2}^{2}\right)\left(M_{2}^{2}-M_{1}^{2}\right)}, \\
D_{2}\left(m_{1}, m_{2}, M_{1}, M_{2}\right)= & \frac{m_{2}^{4} \log \left(\frac{m_{2}^{2}}{m_{1}^{2}}\right)}{\left(m_{2}^{2}-m_{1}^{2}\right)\left(m_{2}^{2}-M_{1}^{2}\right)\left(m_{2}^{2}-M_{2}^{2}\right)} \\
& +\frac{M_{1}^{4} \log \left(\frac{M_{1}^{2}}{m_{1}^{2}}\right)}{\left(M_{1}^{2}-m_{1}^{2}\right)\left(M_{1}^{2}-m_{2}^{2}\right)\left(M_{1}^{2}-M_{2}^{2}\right)} \\
& +\frac{M_{2}^{4} \log \left(\frac{M_{2}^{2}}{m_{1}^{2}}\right)}{\left(M_{2}^{2}-m_{1}^{2}\right)\left(M_{2}^{2}-m_{2}^{2}\right)\left(M_{2}^{2}-M_{1}^{2}\right)},
\end{aligned}
$$

and for $i=1,2$

$$
\begin{aligned}
D_{i}\left(m_{1}, M_{1}, M_{2}\right) & =\lim _{m_{2} \rightarrow m_{1}} D_{i}\left(m_{1}, m_{2}, M_{1}, M_{2}\right), \\
D_{i}\left(m_{1}, M_{1}\right) & =\lim _{M_{2} \rightarrow M_{1}} D_{i}\left(m_{1}, M_{1}, M_{2}\right), \\
\bar{D}_{2}\left(m_{1}, M_{1}, M_{2}\right) & =D_{2}\left(m_{1}, M_{1}, M_{2}\right)-D_{2}\left(0, M_{1}, M_{2}\right) .
\end{aligned}
$$

\section{Random parameter generation and running}

In order to find sample parameter points, we generate random fundamental Yukawa couplings with $y_{i j}^{u, d}=\left|y_{i j}^{u, d}\right| e^{i \phi_{i j}^{u, d}}$ and $\left|y_{i j}^{u, d}\right| \in[0.5,1.5]$ and $\phi_{i j}^{u, d} \in[0,2 \pi]$. The effective Yukawa couplings (2.7) have to reproduce the quark masses and Wolfenstein parameters in table 6 in appendix D. To this end we perform a $\chi^{2}$ fit, with symmetrized $2 \sigma$ errors and require $\chi^{2}<10$.

In order to obtain the new contributions to $K-\bar{K}$ and $B_{s, d}-\bar{B}_{s, d}$ mixing we compute the Wilson coefficients with these effective Yukawas, including the tree-level (5.2) and 
one loop Wilson coefficients given in appendix B. These Wilson coefficients are at the high scale $\mu=m_{h}$ and $\mu=M_{H}, M_{H^{ \pm}}, M_{A}$, respectively. The next step is running the Wilson coefficients from the electroweak scale to the scale at which the matrix elements are evaluated, $\mu=2 \mathrm{GeV}$ in the case of $K-\bar{K}$ mixing and $\mu=m_{b}$ in the case of $B_{s, d}-\bar{B}_{s, d}$ mixing. For $K-\bar{K}$ mixing we use [41]

$$
\left\langle\bar{K}\left|\mathcal{H}_{\mathrm{eff}}^{\Delta S=2}\right| K\right\rangle_{i}=\sum_{j=1}^{5} \sum_{r=1}^{5}\left(b_{j}^{(r, i)}+\eta c_{j}^{(r, i)}\right) \eta^{a_{j}} C_{i}^{s d}(\mu) B_{i}^{K}\left\langle\bar{K}\left|Q_{r}^{s d}\right| K\right\rangle,
$$

in which $\eta=\alpha_{s}(\mu) / \alpha_{s}\left(m_{t}\right), a_{i}, b_{j}^{(r, i)}$ and $c_{j}^{(r, i)}$ are "magic numbers" collected in [39] and $B_{K}^{i}$ are the $B$ parameters collected in table 8 . The matrix elements are given by

$$
\begin{aligned}
\left\langle\bar{K}\left|Q_{1}^{s d}\right| K\right\rangle & =\frac{1}{3} M_{K} f_{k}^{2}, \\
\left\langle\bar{K}\left|Q_{r}^{s d}\right| K\right\rangle & =N_{r}\left(\frac{M_{K}}{m_{d}+m_{s}}\right)^{2} M_{K} f_{k}^{2},
\end{aligned}
$$

with $N_{r}=(-5 / 24,1 / 24,1 / 4,1 / 12)$ for $r=(2,3,4,5)$ and $M_{K}$ and $m_{d}+m_{s}$ again given in table 8. For $B_{d, s}-\bar{B}_{d, s}$ mixing, (C.1) and (C.2) hold with the obvious replacements. The corresponding "magic numbers" can be found in [41], and all other parameters in table 9.

\section{Numerical input}

In this appendix we collect the numerical input used throughout this paper, quark masses and Wolfenstein parameters in table 6 , coupling constants and vector boson masses in

\begin{tabular}{|c|c|c|}
\hline \multicolumn{3}{|c|}{ Wolfenstein Parameters [90] } \\
\hline$\lambda$ & 0.22551 & \pm 0.00091 \\
\hline$A$ & 0.813 & \pm 0.035 \\
\hline $\bar{\eta}$ & 0.342 & \pm 0.024 \\
\hline $\bar{\rho}$ & 0.149 & \pm 0.033 \\
\hline
\end{tabular}
table 7 and parameters relevant for $K-\bar{K}$ and $B_{d, s}-\bar{B}_{d, s}$ mixing in table 8 and table 9 .

\begin{tabular}{|lll|}
\hline \multicolumn{3}{|c|}{ Quark Masses in $\mathrm{GeV}$} \\
\hline$m_{u}\left(m_{Z}\right)$ & 0.00127 & \pm 0.0005 \\
$m_{d}\left(m_{Z}\right)$ & 0.0029 & \pm 0.0012 \\
$m_{s}\left(m_{Z}\right)$ & 0.055 & \pm 0.016 \\
$m_{c}\left(m_{Z}\right)$ & 0.619 & \pm 0.084 \\
$m_{b}\left(m_{Z}\right)$ & 2.89 & \pm 0.09 \\
$m_{t}\left(m_{Z}\right)$ & 171.7 & \pm 3.0 \\
\hline
\end{tabular}

Table 6. Quark masses and Wolfenstein parameters at the electroweak scale. Errors are symmetrized. 


\begin{tabular}{|cl|}
\hline \multicolumn{2}{|c|}{ Couplings and Boson Masses } \\
\hline \multicolumn{2}{|c|}{$[32,91]$} \\
$\alpha_{e}\left(m_{Z}\right)$ & $1 / 127.9$ \\
$\alpha_{s}\left(m_{Z}\right)$ & $0.1185 \pm 0.0006$ \\
$m_{Z}$ & $91.1876 \pm 0.0021 \mathrm{GeV}$ \\
$m_{W}$ & $80.385 \pm 0.015 \mathrm{GeV}$ \\
$G_{F}$ & $1.16638 \cdot 10^{-5} \mathrm{GeV}^{-2}$ \\
\hline
\end{tabular}

Table 7. Gauge boson masses and couplings.

\begin{tabular}{|cl|}
\hline \multicolumn{2}{|c|}{ Parameters in $K-\bar{K}$ mixing $\quad[32,92]$} \\
\hline$B_{1}^{K}$ & $0.537 \pm 0.007 \pm 0.024$ \\
$B_{2}^{K}$ & $0.620 \pm 0.004 \pm 0.031$ \\
$B_{3}^{K}$ & $0.433 \pm 0.003 \pm 0.019$ \\
$B_{4}^{K}$ & $1.081 \pm 0.006 \pm 0.048$ \\
$B_{5}^{K}$ & $0.853 \pm 0.006 \pm 0.049$ \\
$f_{k}$ & $156.2 \pm 0.2 \pm 0.6 \pm 0.3 \mathrm{MeV}$ \\
$M_{K}$ & $497.614 \pm 0.024 \mathrm{MeV}$ \\
$m_{s}+m_{d}$ & $135 \pm 18 \mathrm{MeV}$ \\
\hline
\end{tabular}

Table 8. Parameters relevant for $K-\bar{K}$ mixing.

\begin{tabular}{|c|c|}
\hline Paramete & rs in $B_{d}-\bar{B}_{d}$ mixing $\quad$ [93-95] \\
\hline$B_{1}^{d}$ & $0.85 \pm 0.03 \pm 0.02$ \\
\hline$B_{2}^{d}$ & $0.73 \pm 0.03 \pm 0.01$ \\
\hline$B_{3}^{d}$ & $0.88 \pm 0.12 \pm 0.06$ \\
\hline$B_{4}^{d}$ & $0.95 \pm 0.04 \pm 0.03$ \\
\hline$B_{5}^{d}$ & $1.47 \pm 0.08 \pm 0.09$ \\
\hline$f_{B_{d}}$ & $186 \pm 4 \mathrm{MeV}$ \\
\hline$M_{B_{d}}$ & $5.27942 \pm 0.00012 \mathrm{GeV}$ \\
\hline$m_{b}+m_{d}$ & $4.29 \pm 0.09 \pm 0.08 \pm 0.02 \mathrm{GeV}$ \\
\hline
\end{tabular}

\begin{tabular}{|cl|}
\hline Parameters in $B_{s}-\bar{B}_{s}$ mixing & [93-95] \\
\hline$B_{1}^{s}$ & $0.86 \pm 0.03 \pm 0.01$ \\
$B_{2}^{s}$ & $0.73 \pm 0.03 \pm 0.01$ \\
$B_{3}^{s}$ & $0.89 \pm 0.10 \pm 0.07$ \\
$B_{4}^{s}$ & $0.93 \pm 0.04 \pm 0.01$ \\
$B_{5}^{s}$ & $1.57 \pm 0.07 \pm 0.08$ \\
$f_{B_{s}}$ & $224 \pm 5 \mathrm{MeV}$ \\
$M_{B_{s}}$ & $5.36668 \pm 0.00024 \mathrm{GeV}$ \\
$m_{b}+m_{s}$ & $4.38 \pm 0.09 \pm 0.08 \pm 0.02 \mathrm{GeV}$ \\
\hline
\end{tabular}

Table 9. Parameters relevant for $B_{d, s}-\bar{B}_{d, s}$ mixing. 


\section{E Branching ratios}

$1 \mathrm{a}$

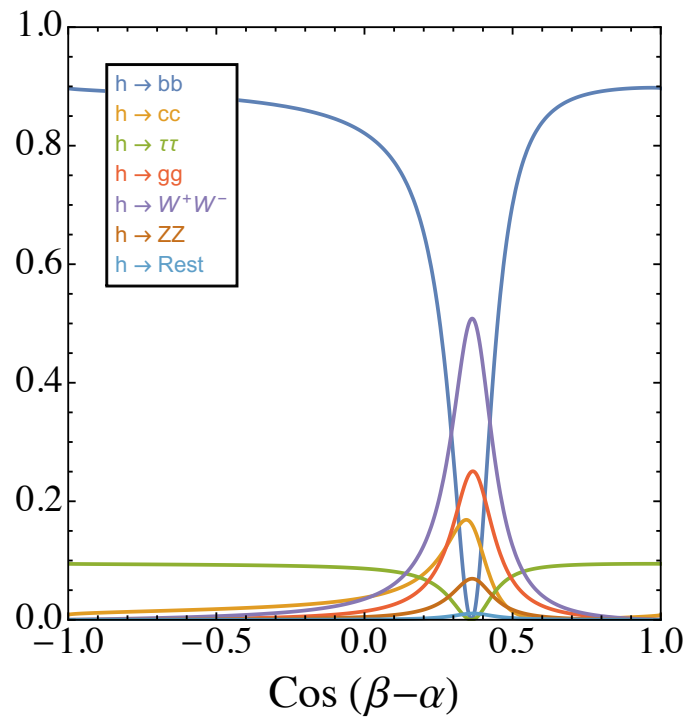

la

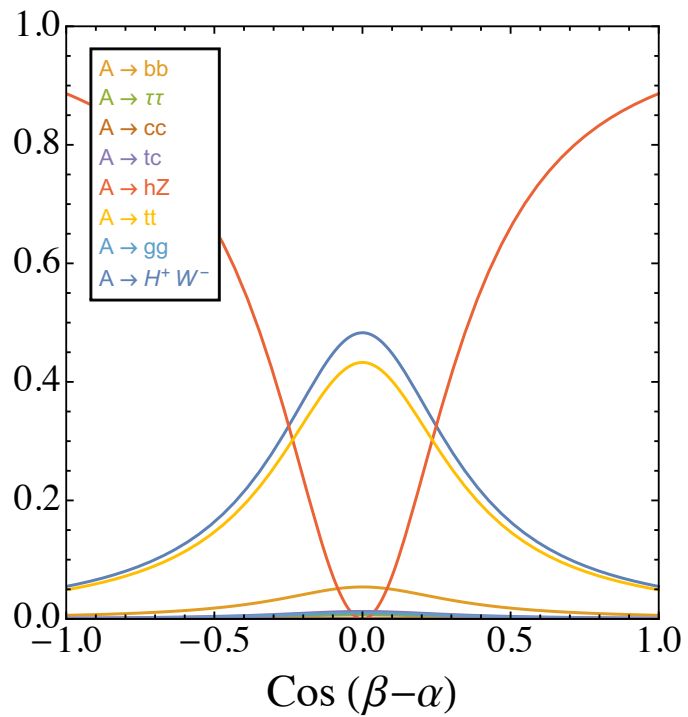

la

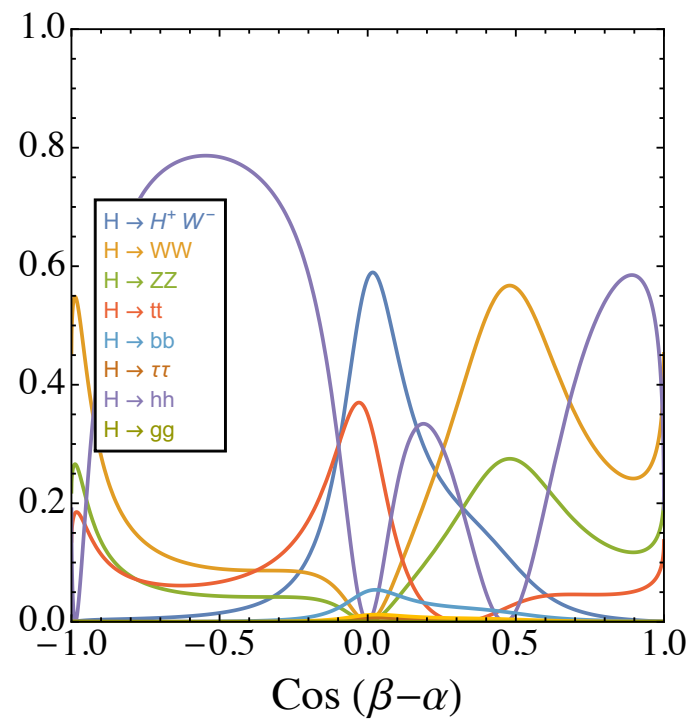

$1 \mathrm{a}$

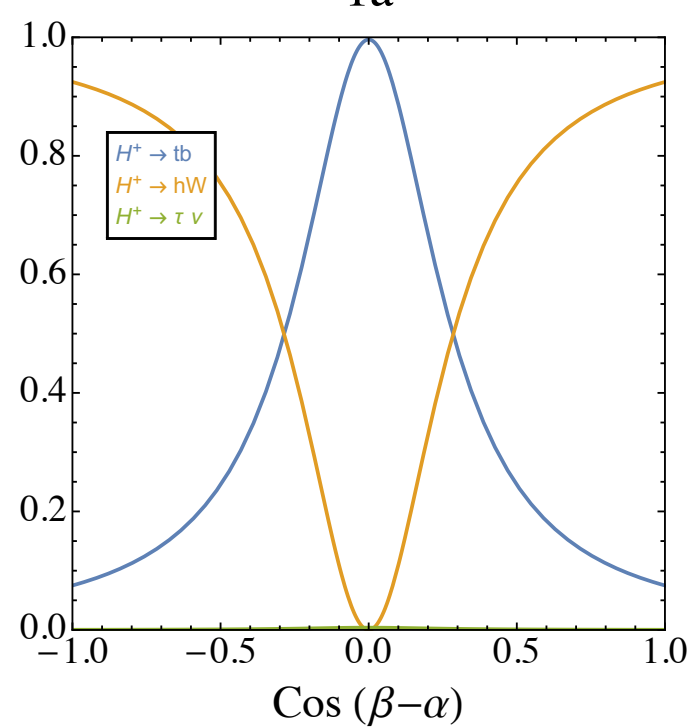

Figure 23. Branching ratios as a function of $\cos (\beta-\alpha)$ for the light neutral scalar (upper left panel), heavy neutral scalar (upper right panel), pseudoscalar (lower left panel) and charged scalar (lower right panel) for the scalar masses and $\tan \beta$ of the benchmark scenario 1a defined in table 4 . 
$1 b$

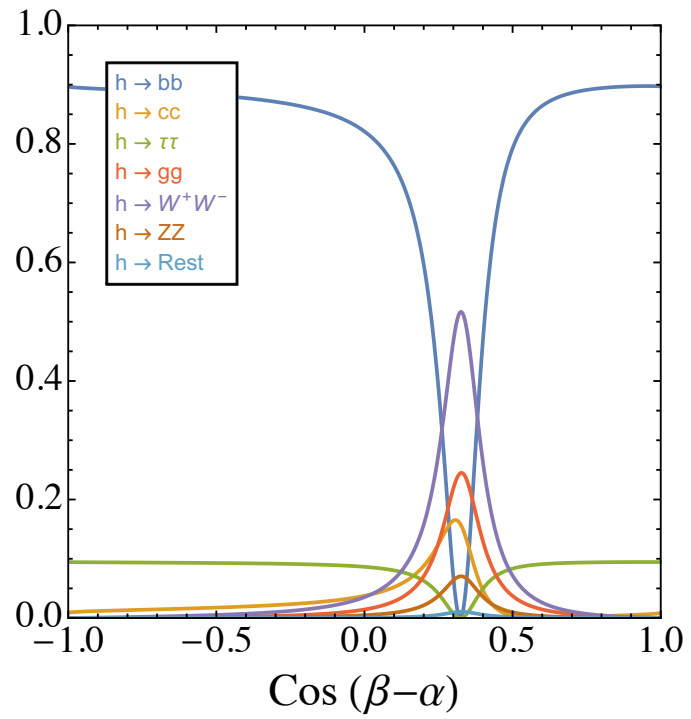

$1 b$

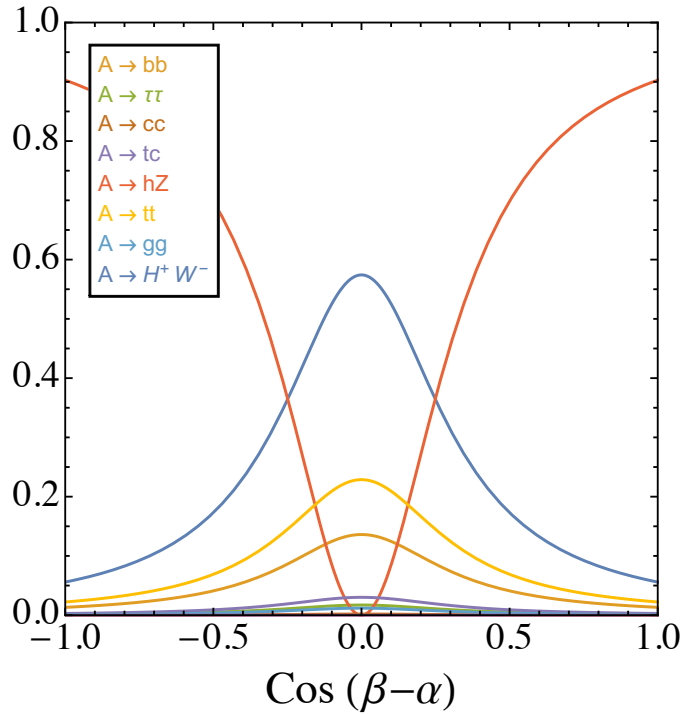

$1 b$

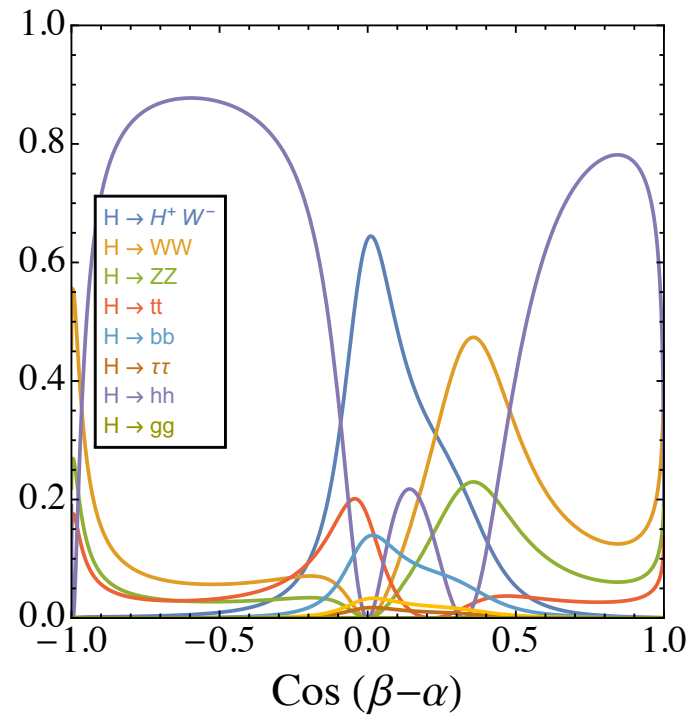

$1 \mathrm{~b}$

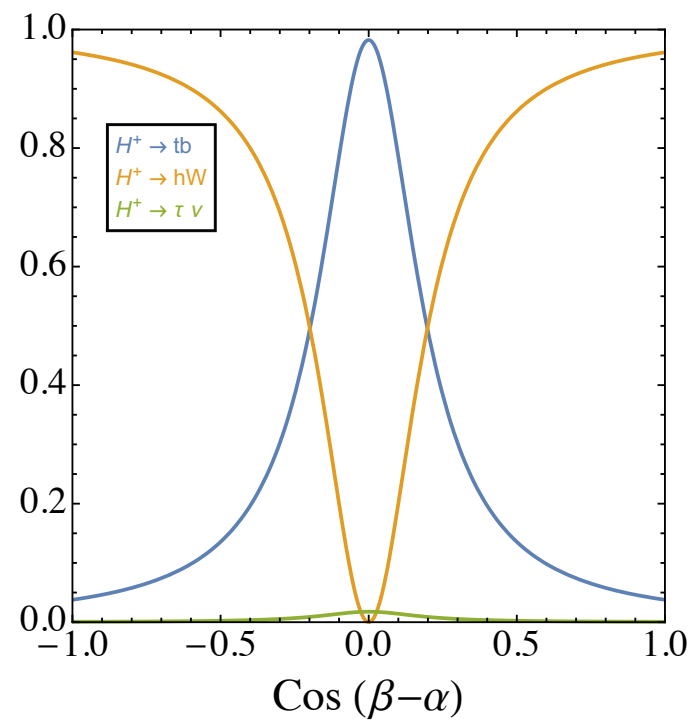

Figure 24. Branching ratios as a function of $\cos (\beta-\alpha)$ for the light neutral scalar (upper left panel), heavy neutral scalar (upper right panel), pseudoscalar (lower left panel) and charged scalar (lower right panel) for the scalar masses and $\tan \beta$ of the benchmark scenario $\mathbf{1 b}$ defined in table 4 . 
2

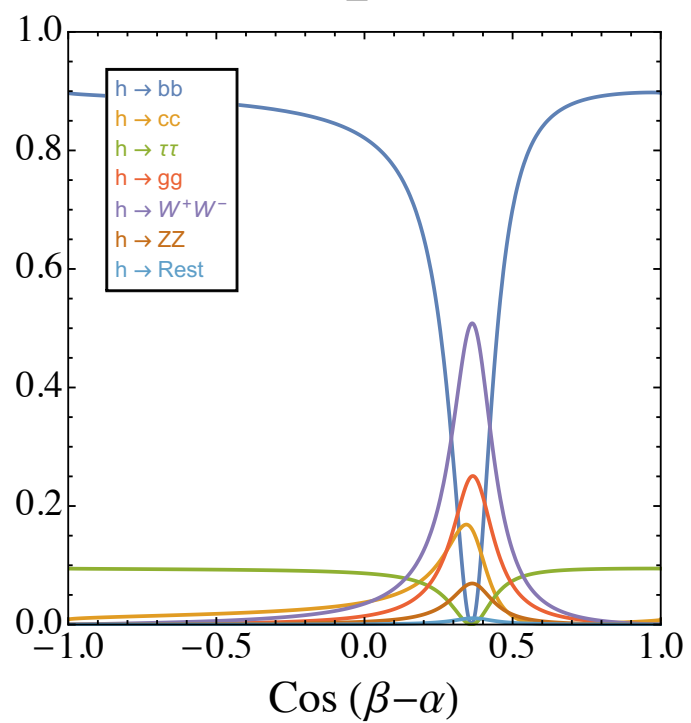

2

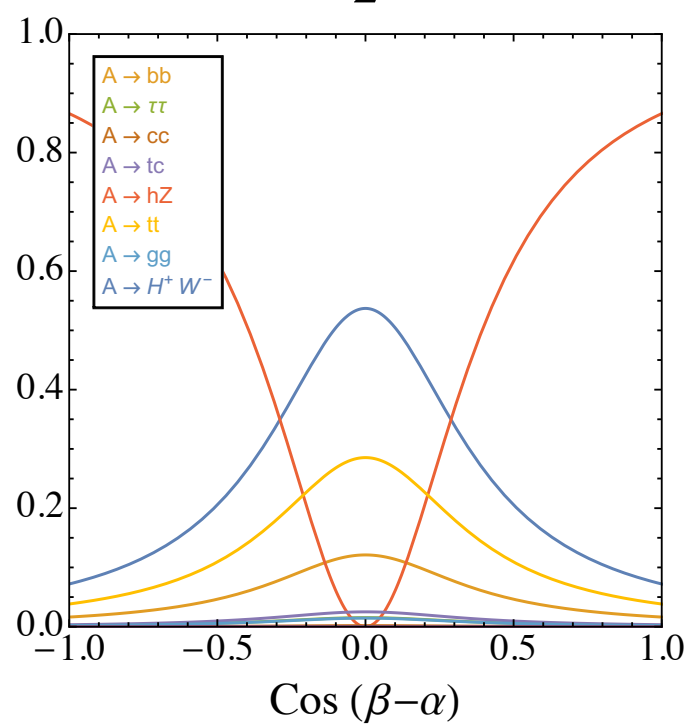

2

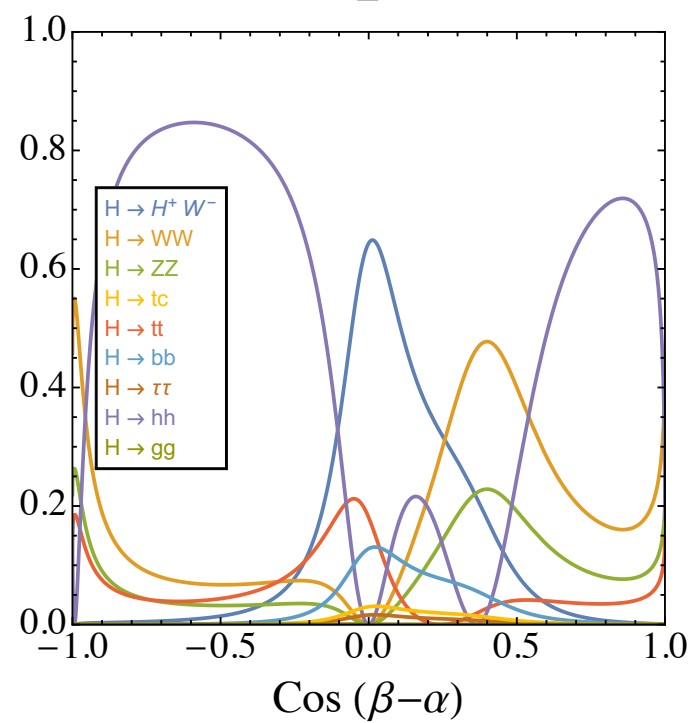

2

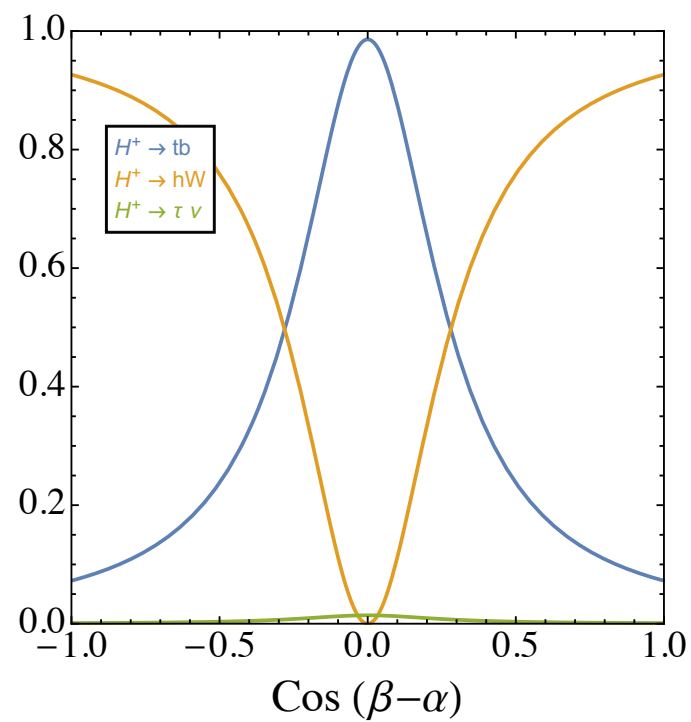

Figure 25. Branching ratios as a function of $\cos (\beta-\alpha)$ for the light neutral scalar (upper left panel), heavy neutral scalar (upper right panel), pseudoscalar (lower left panel) and charged scalar (lower right panel) for the scalar masses and $\tan \beta$ of the benchmark scenario 2 defined in table 5 . 
Open Access. This article is distributed under the terms of the Creative Commons Attribution License (CC-BY 4.0), which permits any use, distribution and reproduction in any medium, provided the original author(s) and source are credited.

\section{References}

[1] C.D. Froggatt and H.B. Nielsen, Hierarchy of Quark Masses, Cabibbo Angles and CP-violation, Nucl. Phys. B 147 (1979) 277 [inSPIRE].

[2] K.S. Babu and S. Nandi, Natural fermion mass hierarchy and new signals for the Higgs boson, Phys. Rev. D 62 (2000) 033002 [hep-ph/9907213] [INSPIRE].

[3] G.F. Giudice and O. Lebedev, Higgs-dependent Yukawa couplings, Phys. Lett. B 665 (2008) 79 [arXiv: 0804.1753] [inSPIRE].

[4] J.F. Gunion, H.E. Haber, G.L. Kane and S. Dawson, The Higgs Hunter's Guide, Front. Phys. 80 (2000) 1 [INSPIRE].

[5] N. Craig, J. Galloway and S. Thomas, Searching for Signs of the Second Higgs Doublet, arXiv:1305.2424 [INSPIRE].

[6] M. Bauer, M. Carena and K. Gemmler, in preparation.

[7] P.M. Ferreira, J.F. Gunion, H.E. Haber and R. Santos, Probing wrong-sign Yukawa couplings at the LHC and a future linear collider, Phys. Rev. D 89 (2014) 115003 [arXiv:1403.4736] [INSPIRE].

[8] B. Dumont, J.F. Gunion, Y. Jiang and S. Kraml, Constraints on and future prospects for Two-Higgs-Doublet Models in light of the LHC Higgs signal, Phys. Rev. D 90 (2014) 035021 [arXiv: 1405.3584] [INSPIRE].

[9] J.F. Gunion and H.E. Haber, The CP conserving two Higgs doublet model: The approach to the decoupling limit, Phys. Rev. D 67 (2003) 075019 [hep-ph/0207010] [INSPIRE].

[10] D.M. Asner et al., ILC Higgs White Paper, arXiv:1310.0763 [INSPIRE].

[11] H.E. Haber, The Higgs data and the Decoupling Limit, arXiv:1401.0152 [INSPIRE].

[12] M. Carena, I. Low, N.R. Shah and C.E.M. Wagner, Impersonating the Standard Model Higgs Boson: Alignment without Decoupling, JHEP 04 (2014) 015 [arXiv: 1310.2248] [INSPIRE].

[13] G.C. Branco, P.M. Ferreira, L. Lavoura, M.N. Rebelo, M. Sher and J.P. Silva, Theory and phenomenology of two-Higgs-doublet models, Phys. Rept. 516 (2012) 1 [arXiv:1106.0034] [INSPIRE].

[14] ATLAS collaboration, Measurements of the Higgs boson production and decay rates and coupling strengths using pp collision data at $\sqrt{s}=7$ and $8 \mathrm{TeV}$ in the ATLAS experiment, ATLAS-CONF-2015-007 (2015).

[15] CMS collaboration, J. Bendavid, Scalar: Cms run 1 final results, prospectives for run 2 (and hl-lhc), talk by J. Bendavid at the 50th Rencontres de Moriond EW 2015, https://indico.in2p3.fr/event/10819/session/3/contribution/112/material/slides/0.pdf.

[16] W. Altmannshofer, S. Gori and G.D. Kribs, A Minimal Flavor Violating 2HDM at the LHC, Phys. Rev. D 86 (2012) 115009 [arXiv:1210.2465] [inSPIRE].

[17] ATLAS collaboration, Observation and measurement of Higgs boson decays to $W W^{*}$ with the ATLAS detector, Phys. Rev. D 92 (2015) 012006 [arXiv:1412.2641] [INSPIRE]. 
[18] CMS collaboration, Measurement of Higgs boson production and properties in the $W W$ decay channel with leptonic final states, JHEP 01 (2014) 096 [arXiv:1312.1129] [INSPIRE].

[19] ATLAS collaboration, Measurements of Higgs boson production and couplings in the four-lepton channel in pp collisions at center-of-mass energies of 7 and $8 \mathrm{TeV}$ with the ATLAS detector, Phys. Rev. D 91 (2015) 012006 [arXiv:1408.5191] [INSPIRE].

[20] CMS collaboration, Measurement of the properties of a Higgs boson in the four-lepton final state, Phys. Rev. D 89 (2014) 092007 [arXiv:1312.5353] [InSPIRE].

[21] ATLAS collaboration, Measurement of Higgs boson production in the diphoton decay channel in pp collisions at center-of-mass energies of 7 and $8 \mathrm{TeV}$ with the ATLAS detector, Phys. Rev. D 90 (2014) 112015 [arXiv:1408.7084] [INSPIRE].

[22] CMS collaboration, Observation of the diphoton decay of the Higgs boson and measurement of its properties, Eur. Phys. J. C 74 (2014) 3076 [arXiv:1407.0558] [INSPIRE].

[23] ATLAS collaboration, Search for the Standard Model Higgs boson produced in association with top quarks and decaying into bb in pp collisions at $\sqrt{s}=8 \mathrm{TeV}$ with the ATLAS detector, Eur. Phys. J. C 75 (2015) 349 [arXiv:1503.05066] [INSPIRE].

[24] ATLAS collaboration, Search for the b $\bar{b}$ decay of the Standard Model Higgs boson in associated $(W / Z) H$ production with the ATLAS detector, JHEP 01 (2015) 069 [arXiv:1409.6212] [INSPIRE].

[25] CMS Collaboration, Search for ttH production using the Matrix Element Method, CMS-PAS-HIG-14-010 (2014).

[26] CMS collaboration, Search for the standard model Higgs boson produced in association with a W or a Z boson and decaying to bottom quarks, Phys. Rev. D 89 (2014) 012003 [arXiv: 1310.3687] [INSPIRE].

[27] ATLAS collaboration, Evidence for the Higgs-boson Yukawa coupling to tau leptons with the ATLAS detector, JHEP 04 (2015) 117 [arXiv: 1501.04943] [INSPIRE].

[28] CMS collaboration, Evidence for the $125 \mathrm{GeV}$ Higgs boson decaying to a pair of $\tau$ leptons, JHEP 05 (2014) 104 [arXiv: 1401.5041] [INSPIRE].

[29] M. Carena, I. Low and C.E.M. Wagner, Implications of a Modified Higgs to Diphoton Decay Width, JHEP 08 (2012) 060 [arXiv:1206.1082] [INSPIRE].

[30] A. Denner, S. Heinemeyer, I. Puljak, D. Rebuzzi and M. Spira, Standard Model Higgs-Boson Branching Ratios with Uncertainties, Eur. Phys. J. C 71 (2011) 1753 [arXiv:1107.5909] [INSPIRE].

[31] P.R. Archer, M. Carena, A. Carmona and M. Neubert, Higgs Production and Decay in Models of a Warped Extra Dimension with a Bulk Higgs, JHEP 01 (2015) 060 [arXiv: 1408.5406] [INSPIRE].

[32] Particle Data Group collaboration, K. Olive et al., Review of Particle Physics, Chin. Phys. C 38 (2014) 090001.

[33] M. Carena, H.E. Haber, I. Low, N.R. Shah and C.E.M. Wagner, Complementarity between nonstandard Higgs boson searches and precision Higgs boson measurements in the MSSM, Phys. Rev. D 91 (2015) 035003 [arXiv:1410.4969] [INSPIRE]. 
[34] A.J. Buras, F. De Fazio, J. Girrbach, R. Knegjens and M. Nagai, The Anatomy of Neutral Scalars with FCNCs in the Flavour Precision Era, JHEP 06 (2013) 111 [arXiv:1303.3723] [INSPIRE].

[35] A.J. Buras, M.V. Carlucci, S. Gori and G. Isidori, Higgs-mediated FCNCs: Natural Flavour Conservation vs. Minimal Flavour Violation, JHEP 10 (2010) 009 [arXiv: 1005.5310] [INSPIRE].

[36] A. Bevan et al., Standard Model updates and new physics analysis with the Unitarity Triangle fit, arXiv: 1411.7233 [INSPIRE].

[37] A.J. Buras, P. Krawczyk, M.E. Lautenbacher and C. Salazar, $B_{0}-\bar{B}_{0}$ Mixing, CP Violation, $K^{+} \rightarrow \pi^{+} \nu \bar{\nu}$ and $B \rightarrow K \gamma X$ in a Two Higgs Doublet Model, Nucl. Phys. B 337 (1990) 284 [INSPIRE].

[38] D. Atwood, L. Reina and A. Soni, Phenomenology of two Higgs doublet models with flavor changing neutral currents, Phys. Rev. D 55 (1997) 3156 [hep-ph/9609279] [INSPIRE].

[39] M. Ciuchini et al., $\Delta M_{K}$ and $\epsilon_{K}$ in SUSY at the next-to-leading order, JHEP 10 (1998) 008 [hep-ph/9808328] [INSPIRE].

[40] J. Laiho, E. Lunghi and R.S. Van de Water, Lattice QCD inputs to the CKM unitarity triangle analysis, Phys. Rev. D 81 (2010) 034503 [arXiv:0910.2928] [INSPIRE].

[41] UTfit collaboration, M. Bona et al., Model-independent constraints on $\Delta F=2$ operators and the scale of new physics, JHEP 03 (2008) 049 [arXiv:0707.0636] [INSPIRE].

[42] M. Jung, A. Pich and P. Tuzon, Charged-Higgs phenomenology in the Aligned two-Higgs-doublet model, JHEP 11 (2010) 003 [arXiv:1006.0470] [INSPIRE].

[43] A. Crivellin, A. Kokulu and C. Greub, Flavor-phenomenology of two-Higgs-doublet models with generic Yukawa structure, Phys. Rev. D 87 (2013) 094031 [arXiv:1303.5877] [INSPIRE].

[44] T. Hermann, M. Misiak and M. Steinhauser, $\bar{B} \rightarrow X_{s} \gamma$ in the Two Higgs Doublet Model up to Next-to-Next-to-Leading Order in QCD, JHEP 11 (2012) 036 [arXiv:1208.2788] [INSPIRE].

[45] M. Misiak et al., Updated NNLO QCD predictions for the weak radiative B-meson decays, Phys. Rev. Lett. 114 (2015) 221801 [arXiv: 1503.01789] [INSPIRE].

[46] J.A. Aguilar-Saavedra, Top flavor-changing neutral interactions: Theoretical expectations and experimental detection, Acta Phys. Polon. B 35 (2004) 2695 [hep-ph/0409342] [INSPIRE].

[47] S. Casagrande, F. Goertz, U. Haisch, M. Neubert and T. Pfoh, Flavor Physics in the Randall-Sundrum Model: I. Theoretical Setup and Electroweak Precision Tests, JHEP 10 (2008) 094 [arXiv: 0807.4937] [INSPIRE].

[48] ATLAS collaboration, Search for top quark decays $t \rightarrow q H$ with $H \rightarrow \gamma \gamma$ using the ATLAS detector, JHEP 06 (2014) 008 [arXiv:1403.6293] [INSPIRE].

[49] CMS collaboration, Searches for heavy Higgs bosons in two-Higgs-doublet models and for $t \rightarrow$ ch decay using multilepton and diphoton final states in pp collisions at $8 \mathrm{TeV}$, Phys. Rev. D 90 (2014) 112013 [arXiv:1410.2751] [INSPIRE].

[50] Top Quark Working Group collaboration, K. Agashe et al., Working Group Report: Top Quark, arXiv:1311.2028 [INSPIRE].

[51] D. Eriksson, J. Rathsman and O. Stal, 2HDMC: Two-Higgs-Doublet Model Calculator Physics and Manual, Comput. Phys. Commun. 181 (2010) 189 [arXiv:0902.0851] [InSPIRE]. 
[52] H.E. Haber and D. O'Neil, Basis-independent methods for the two-Higgs-doublet model III: The CP-conserving limit, custodial symmetry and the oblique parameters S, T, U, Phys. Rev. D 83 (2011) 055017 [arXiv: 1011.6188] [INSPIRE].

[53] A. Celis, V. Ilisie and A. Pich, LHC constraints on two-Higgs doublet models, JHEP 07 (2013) 053 [arXiv: 1302.4022] [InSPIRE].

[54] Gfitter Group collaboration, M. Baak et al., The global electroweak fit at NNLO and prospects for the LHC and ILC, Eur. Phys. J. C 74 (2014) 3046 [arXiv:1407.3792] [INSPIRE].

[55] A.J. Buras, K. Gemmler and G. Isidori, Quark flavour mixing with right-handed currents: an effective theory approach, Nucl. Phys. B 843 (2011) 107 [arXiv:1007.1993] [INSPIRE].

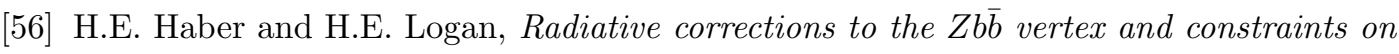
extended Higgs sectors, Phys. Rev. D 62 (2000) 015011 [hep-ph/9909335] [INSPIRE].

[57] H.E. Logan, Radiative corrections to the $Z$ b anti-b vertex and constraints on extended Higgs sectors, hep-ph/9906332 [INSPIRE].

[58] B. Batell, S. Gori and L.-T. Wang, Higgs Couplings and Precision Electroweak Data, JHEP 01 (2013) 139 [arXiv: 1209.6382] [INSPIRE].

[59] M. Spira, HIGLU: A program for the calculation of the total Higgs production cross-section at hadron colliders via gluon fusion including QCD corrections, hep-ph/9510347 [INSPIRE].

[60] A. Djouadi, Higgs particles at future hadron and electron-positron colliders, Int. J. Mod. Phys. A 10 (1995) 1 [hep-ph/9406430] [INSPIRE].

[61] A. Djouadi, J. Kalinowski and P.M. Zerwas, Two and three-body decay modes of SUSY Higgs particles, Z. Phys. C 70 (1996) 435 [hep-ph/9511342] [InSPIRE].

[62] LHC Higgs Cross section Working Group collaboration, S. Dittmaier et al., Handbook of LHC Higgs Cross sections: 1. Inclusive Observables, arXiv:1101.0593 [INSPIRE].

[63] LHC Higgs Cross section Working Group collaboration, J.R. Andersen et al., Handbook of LHC Higgs Cross sections: 3. Higgs Properties, arXiv:1307.1347 [INSPIRE].

[64] M. Flechl, R. Klees, M. Krämer, M. Spira and M. Ubiali, Improved cross-section predictions for heavy charged Higgs boson production at the LHC, Phys. Rev. D 91 (2015) 075015 [arXiv:1409.5615] [INSPIRE].

[65] ATLAS collaboration, Search for a CP-odd Higgs boson decaying to Zh in pp collisions at $\sqrt{s}=8 \mathrm{TeV}$ with the ATLAS detector, Phys. Lett. B 744 (2015) 163 [arXiv:1502.04478] [INSPIRE].

[66] CMS collaboration, Search for a pseudoscalar boson decaying into a $Z$ boson and the 125 GeV Higgs boson in $\ell^{+} \ell^{-} b \bar{b}$ final states, Phys. Lett. B 748 (2015) 221 [arXiv: 1504.04710] [INSPIRE].

[67] S. Dittmaier et al., Handbook of LHC Higgs Cross sections: 2. Differential Distributions, arXiv: 1201.3084 [INSPIRE].

[68] CMS collaboration, Search for a Higgs Boson in the Mass Range from 145 to $1000 \mathrm{GeV}$ Decaying to a Pair of $W$ or $Z$ Bosons, arXiv: 1504.00936 [INSPIRE].

[69] CMS collaboration, Update of the search for the Standard Model Higgs boson decaying into $W W$ in the vector boson fusion production channel, CMS-PAS-HIG-13-022 (2013). 
[70] CMS collaboration, Search for charged Higgs bosons with the H+ to tau nu decay channel in the fully hadronic final state at $\sqrt{s}=8 \mathrm{TeV}$, CMS-PAS-HIG-14-020 (2014).

[71] CMS collaboration, Search for a heavy charged Higgs boson in proton-proton collisions at $\sqrt{s}=8$ TeV with the CMS detector, CMS-PAS-HIG-13-026 (2014).

[72] ATLAS collaboration, Search for charged Higgs bosons decaying via $H^{ \pm} \rightarrow \tau^{ \pm} \nu$ in fully hadronic final states using pp collision data at $\sqrt{s}=8$ TeV with the ATLAS detector, JHEP 03 (2015) 088 [arXiv: 1412.6663] [INSPIRE].

[73] L. Calibbi, Z. Lalak, S. Pokorski and R. Ziegler, Universal Constraints on Low-Energy Flavour Models, JHEP 07 (2012) 004 [arXiv: 1204.1275] [INSPIRE].

[74] ATLAS collaboration, Search for pair and single production of new heavy quarks that decay to a $Z$ boson and a third-generation quark in pp collisions at $\sqrt{s}=8$ TeV with the ATLAS detector, JHEP 11 (2014) 104 [arXiv:1409.5500] [INSPIRE].

[75] CMS collaboration, Search for Vector-Like b' Pair Production with Multilepton Final States in pp collisions at $\sqrt{s}=8$ TeV, CMS-PAS-B2G-13-003 (2013).

[76] CMS collaboration, Inclusive search for a vector-like T quark with charge $\frac{2}{3}$ in pp collisions at $\sqrt{s}=8 \mathrm{TeV}$, Phys. Lett. B 729 (2014) 149 [arXiv:1311.7667] [InSPIRE].

[77] CMS collaboration, Search for vector-like quarks in final states with a single lepton and jets in pp collisions at $\sqrt{s}=8$ TeV, CMS-PAS-B2G-12-017 (2014).

[78] O. Matsedonskyi, G. Panico and A. Wulzer, On the Interpretation of Top Partners Searches, JHEP 12 (2014) 097 [arXiv: 1409.0100] [InSPIRE].

[79] A. Atre, M. Carena, T. Han and J. Santiago, Heavy Quarks Above the Top at the Tevatron, Phys. Rev. D 79 (2009) 054018 [arXiv:0806.3966] [InSPIRE].

[80] A. Atre et al., Model-Independent Searches for New Quarks at the LHC, JHEP 08 (2011) 080 [arXiv: 1102.1987] [INSPIRE].

[81] G.P. Van Onsem and D. Jorgen, Search for new heavy quarks with the CMS detector at the Large Hadron Collider, Ph.D. thesis, Vrije U., Brussels, 2014, CERN-THESIS-2014-108.

[82] G.T. Bodwin, F. Petriello, S. Stoynev and M. Velasco, Higgs boson decays to quarkonia and the Hēc coupling, Phys. Rev. D 88 (2013) 053003 [arXiv:1306.5770] [INSPIRE].

[83] M. König and M. Neubert, Exclusive Radiative Higgs Decays as Probes of Light-Quark Yukawa Couplings, JHEP 08 (2015) 012 [arXiv: 1505.03870] [INSPIRE].

[84] ATLAS collaboration, Search for Higgs and $Z$ Boson Decays to $J / \psi \gamma$ and $\Upsilon(n S) \gamma$ with the ATLAS Detector, Phys. Rev. Lett. 114 (2015) 121801 [arXiv:1501.03276] [INSPIRE].

[85] G. Perez, Y. Soreq, E. Stamou and K. Tobioka, Prospects for measuring the Higgs coupling to light quarks, arXiv:1505.06689 [INSPIRE].

[86] C. Delaunay, T. Golling, G. Perez and Y. Soreq, Enhanced Higgs boson coupling to charm pairs, Phys. Rev. D 89 (2014) 033014 [arXiv:1310.7029] [InSPIRE].

[87] F. Bishara, J. Brod, P. Uttayarat and J. Zupan, Nonstandard Yukawa Couplings and Higgs Portal Dark Matter, arXiv: 1504.04022 [INSPIRE].

[88] A.J. Buras, P.H. Chankowski, J. Rosiek and L. Slawianowska, $\Delta M_{s} / \Delta M_{d}$, $\sin 2 \beta$ and the angle $\gamma$ in the presence of new $\Delta F=2$ operators, Nucl. Phys. B 619 (2001) 434 [hep-ph/0107048] [INSPIRE]. 
[89] Z.-z. Xing, H. Zhang and S. Zhou, Updated Values of Running Quark and Lepton Masses, Phys. Rev. D 77 (2008) 113016 [arXiv:0712.1419] [INSPIRE].

[90] CKMfitter Group collaboration, J. Charles et al., CP violation and the CKM matrix: Assessing the impact of the asymmetric B factories, Eur. Phys. J. C 41 (2005) 1 [hep-ph/0406184] [INSPIRE].

[91] P.J. Mohr, B.N. Taylor and D.B. Newell, CODATA Recommended Values of the Fundamental Physical Constants: 2010, Rev. Mod. Phys. 84 (2012) 1527 [arXiv:1203.5425] [INSPIRE].

[92] SWME collaboration, T. Bae et al., Neutral kaon mixing from new physics: matrix elements in $N_{f}=2+1$ lattice QCD, Phys. Rev. D 88 (2013) 071503 [arXiv:1309.2040] [InSPIRE].

[93] V. Lubicz and C. Tarantino, Flavour physics and Lattice QCD: Averages of lattice inputs for the Unitarity Triangle Analysis, Nuovo Cim. B 123 (2008) 674 [arXiv:0807.4605] [INSPIRE].

[94] ETM collaboration, N. Carrasco et al., B-physics from $N_{f}=2$ tmQCD: the Standard Model and beyond, JHEP 03 (2014) 016 [arXiv:1308.1851] [INSPIRE].

[95] HPQCD collaboration, R.J. Dowdall, C.T.H. Davies, R.R. Horgan, C.J. Monahan and J. Shigemitsu, B-Meson Decay Constants from Improved Lattice Nonrelativistic QCD with Physical u, d, s and c Quarks, Phys. Rev. Lett. 110 (2013) 222003 [arXiv:1302.2644] [INSPIRE]. 